DANTE COAQUIRA BEGAZO

\title{
AVALIAÇÃO OBJETIVA E SUBJETIVA DE QUALIDADE DE VÍDEO VIA REDE IP COM VARIAÇÃO DE ATRASO
}

Dissertação apresentada à Escola Politécnica da Universidade de São Paulo para obtenção do Título de Mestre em Ciências. 

DANTE COAQUIRA BEGAZO

\section{AVALIAÇÃO OBJETIVA E SUBJETIVA DE QUALIDADE DE VÍDEO VIA REDE IP COM VARIAÇÃO DE ATRASO}

Dissertação apresentada à Escola Politécnica da Universidade de São Paulo para obtenção do Título de Mestre em Ciências.

Área de Concentração:

Sistemas Eletrônicos

Orientador:

Prof. Dr. Miguel Arjona Ramírez 
Este exemplar foi revisado e alterado em relação à versão original, sob responsabilidade única do autor e com a anuência de seu orientador.

São Paulo, 03 de dezembro de 2012.

Assinatura do autor

Assinatura do orientador

FICHA CATALOGRÁFICA

Begazo, Dante Coaquira

Avaliação objetiva e subjetiva de qualidade de vídeo via rede IP com variação de atraso / D.C. Begazo. -- ed.rev. -- São Paulo, 2012.

$62 \mathrm{p}$.

Dissertação (Mestrado) - Escola Politécnica da Universidade de São Paulo. Departamento de Engenharia de Sistemas Eletrônicos.

1. Redes de computadores 2 . Redes multimídia 3. Processamento de sinais de vídeo 4. Processamento digital de imagens 5. Vídeo (Qualidade) I. Universidade de São Paulo. Escola Politécnica. Departamento de Engenharia de Sistemas Eletrônicos II. t. 
"Na realidade, todas as coisas, todos os acontecimentos, para quem os sabe ler com profundidade, encerram uma mensagem que, em definitivo, remete para Deus"

João Paulo II. 
Aos meus queridos pais

Mayela e Maximiliano. 


\section{Agradecimentos}

Agradeço primeiramente ao meu orientador Prof. Dr. Miguel Arjona Ramírez pelas orientações e ensinamentos durante minha jornada na Escola Politêcnica.

Aos Profs. Drs. Wagner Luiz Zucchi e Mário Minami pelas sugestões no meu exame de qualificação.

Aos funcionários da secretaria de pós-graduação e demais colaboradores da EPUSP.

Ao CNPq - Conselho Nacional de Desenvolvimento Científico e Tecnológico - e à FAPESP - Fundação de Amparo à Pesquisa do Estado de São Paulo - pelo suporte financeiro.

À SAS - Superintendência de Assistência Social - da USP, especialmente a Carla Cucolo pelo apoio moradia.

Às pessoas que me ajudaram na realização neste trabalho.

Aos meus amigos de Brasil e Perú pela grande amizade e pelos momentos felizes vividos juntos.

Aos meus amigos Demóstenes Zegarra Rodríguez, João Mendes Filho e Renata Lopes Rosa pela valiosa ajuda neste trabalho, e pela grande amizade construída durante todos estes anos.

A Carmen Palacios por todo o carinho, dedicação e apoio dado durante todos estes anos juntos.

Aos meus irmãos Consuelo, Antonieta e Raúl, aos meus sobrinhos Valeria, Luciana e Sebastián e especialmente aos meus pais Mayela e Maximiliano pelo apoio, fortaleza, compreensão, paciência, grande amor e felicidade dados desde sempre.

Por último, agradeço a Deus por ter me dado uma familia linda e amigos verdadeiros. 


\section{Resumo}

Atualmente existe uma grande variedade de serviços de telecomunicações focados na transmissão de voz, vídeo e dados através de redes complexas, embora, em muitos casos, o usuário final não seja atendido com um nível de qualidade aceitável. Neste trabalho, se avalia como o serviço de streaming de vídeo em uma rede com protocolo Internet (IP) pode ser afetado por uma condição adversa da rede, tal como a variação de atraso (jitter). São mostrados os resultados de avaliações objetivas e subjetivas de streaming de vídeo que indicam que a qualidade de vídeo é diretamente afetada por fatores de degradação da rede IP como a variação de atraso de pacotes. Além disso, se verifica que cenas de maior movimento também são mais afetadas pela variação de atraso. Para a realização dos testes, utiliza-se um cenário de emulação de rede isolado, no qual são parametrizadas diferentes condições de rede. Assim, no canal de transmissão são configurados diversos valores de variação de atraso, obtendo-se uma Base de Dados de vídeos com diferentes graus de degradação de qualidade. Estes vídeos são avaliados utilizando métodos subjetivos: Índice por Categorias Absolutas (ACR - Absolute Category Rating) e Índice por Categorias de Degradação (DCR Degradation Category Rating) e métricas objetivas: Relação Sinal-Ruído de Pico (PSNR Peak Signal to Noise Ratio), Índice de Similaridade Estrutural (SSIM - Structural Similarity Index) e Medição de Qualidade de Vídeo (VQM - Video Quality Metric). Com a finalidade de mostrar o desempenho das métricas objetivas em relação às subjetivas são empregados os coeficientes de correlação, além do erro de predição e quadrático médio. Adicionalmente, é importante destacar que a partir dos resultados obtidos são estabelecidos intervalos de valores de variação de atraso para os quais a qualidade do vídeo é considerada aceitável ou não para o usuário final. Finalmente, obtém-se uma Base de Dados de vídeos com diferentes graus de degradação da qualidade e que pode ser utilizada em futuras pesquisas.

Palavras-chave: Qualidade de Vídeo, Streaming de Vídeo, Métricas Objetivas, Métricas Subjetivas, Variação de Atraso. 


\section{Abstract}

Nowadays, there is a wide range of telecommunications services focused on the transmission of voice, video and data across complex networks, although, in many cases, the end user is not satisfied with an acceptable quality level. In this work it is assessed how the video streaming service over an IP network can be affected by adverse conditions on the network, such as packet delay variation (jitter). Results from objective and subjective video streaming assessments are shown and indicate that the video quality is directly affected by IP network degradation factors such as packet delay variation. Beyond that, it is also verified that scenes with great movement content are also more sensitive to packet delay variation. For experimental tests, a network emulation totally isolated is considered, for which different network conditions are parameterized. Then, to the transmission channel, different values of packet delay variation are configured and videos are assessed, using subjective metrics: Absolute Category Rating (ACR) and Degradation Category Rating (DCR), and objective metrics: Peak Signal to Noise Ratio (PSNR), Structural Similarity (SSIM) and Video Quality Metric (VQM). In order to show the performance of objective metrics in relation to subjective ones correlation coefficients are employed as well as the prediction error and the mean square error. Finally, it is important to note that, from the results obtained, it can be established a range of delay variation values in which the video quality is acceptable or not at the end user. Additionally, a Video Data Base is obtained with different degrees of quality degradation and which can be used for future researches.

Keywords: Video Quality, Video Streaming, Objective Metrics, Subjective Metrics, Delay Variation. 


\section{Sumário}

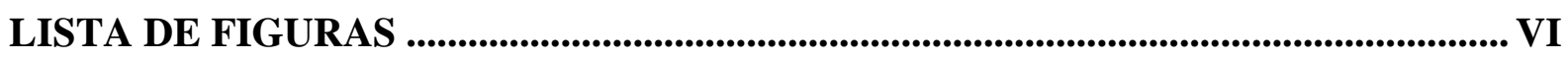

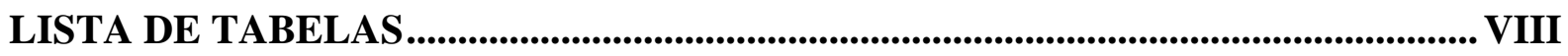

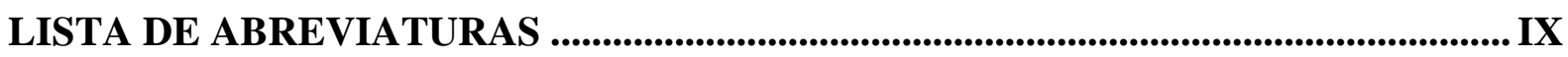

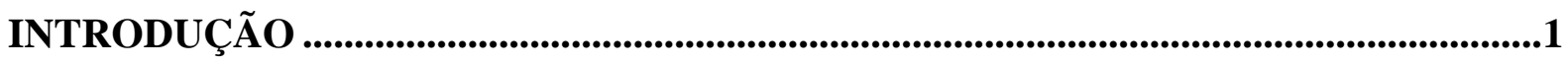

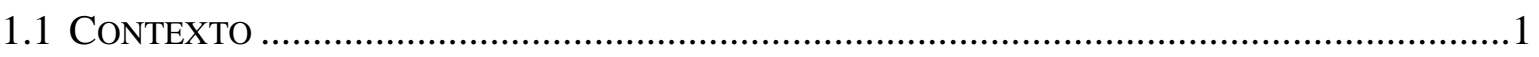

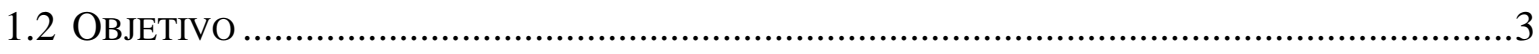

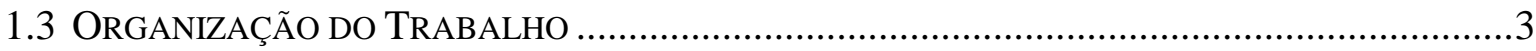

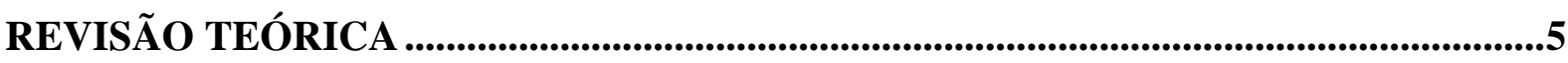

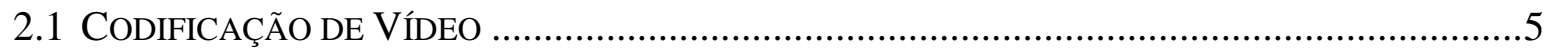

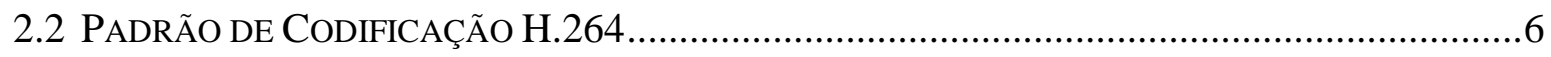

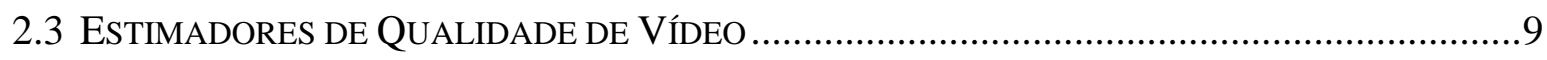

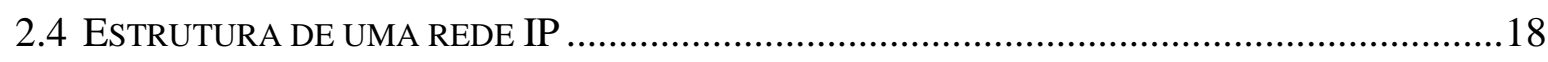

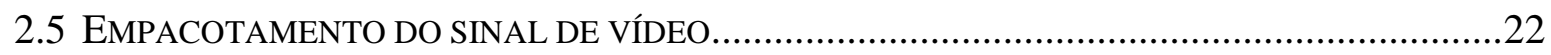

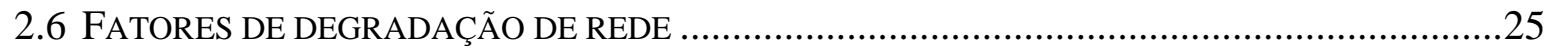

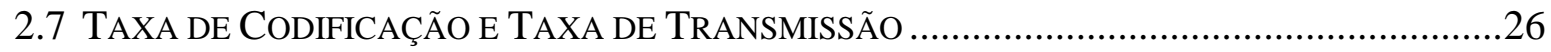

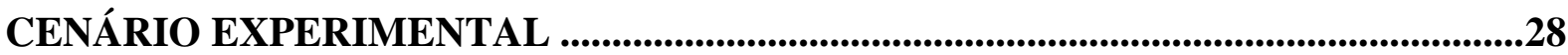

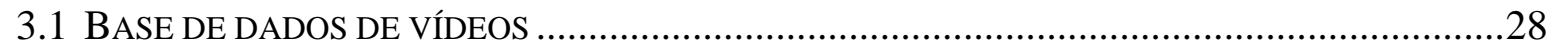

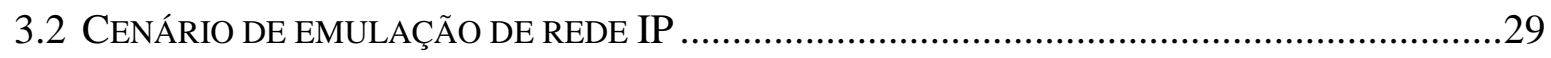

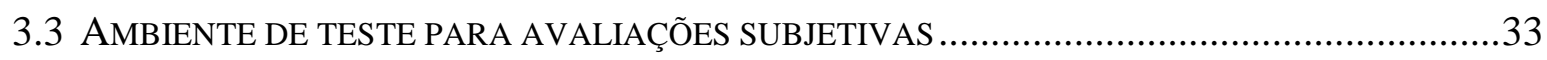

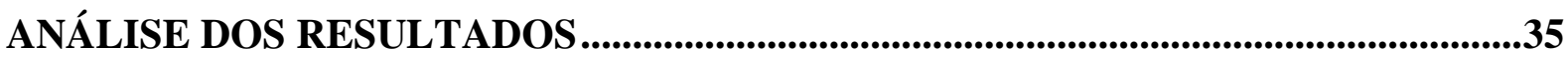

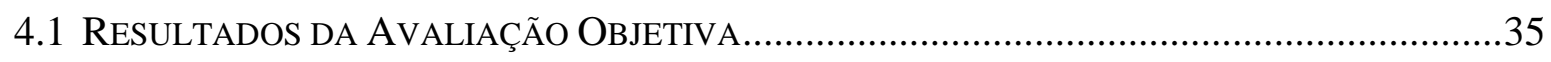

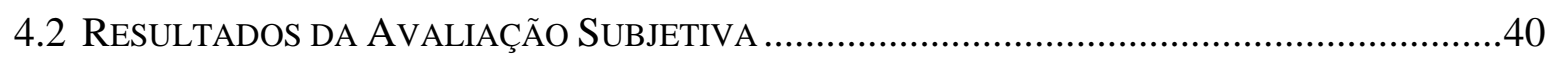

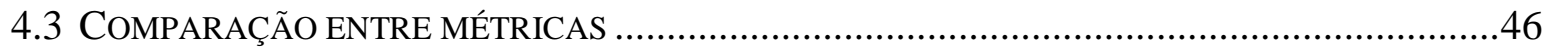


CONCLUSÕES E TRABALHOS FUTUROS..............................................................51

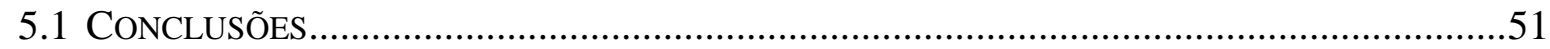

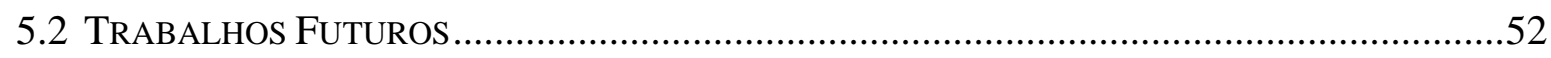

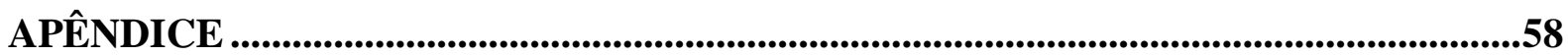

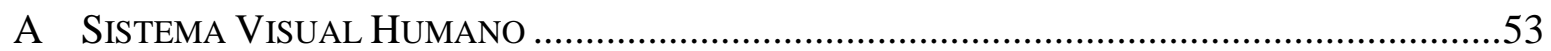

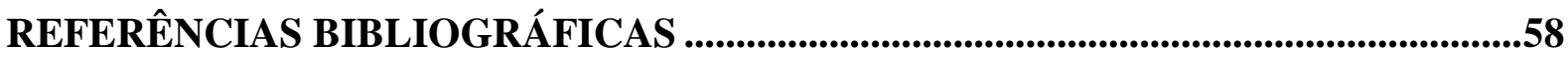




\section{Lista de Figuras}

2.1 Diagrama de blocos do algoritmo da métrica objetiva VQM....................................13

2.2 Escala de classificação para a métrica subjetiva DSIS ...............................................16

2.3 Escala de classificação para a métrica subjetiva DSCQS.............................................18

2.4 Arquitetura Cliente-servidor: Download-and-play....................................................19

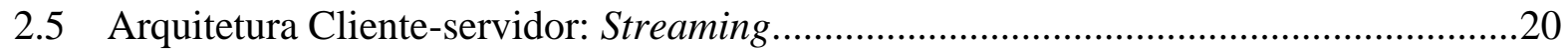

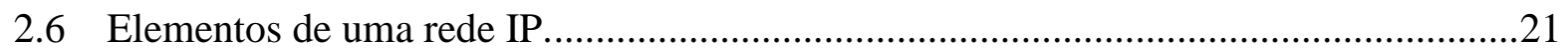

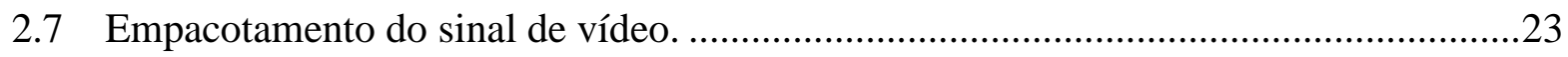

2.8 Protocolos usados na transmissão do vídeo codificado em uma rede IP......................24

2.9 Tamanho de cabeçalhos em uma rede Ethernet .......................................................26

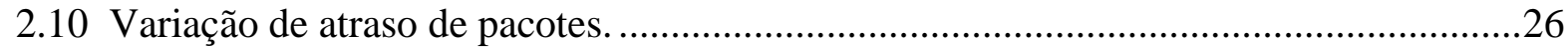

3.1 Imagens dos vídeos originais de Akiyo, Foreman e Football.....................................29

3.2 Configuração do cenário de emulação de rede IP proposto com ferramentas................30

3.3 Configuração lógica de endereçamento IP para o cenário de emulação proposto..........30

3.4 Vídeo degradado de Akiyo com variação de atraso de 0,4,8,12,16 e 20 ms. ................31

3.5 Vídeo degradado de Foreman com variação de atraso de 0,4,8,12,16 e 20 ms.............31

3.6 Vídeo degradado de Football com variação de atraso de 0,4,8,12,16 e 20 ms..............32

3.7 Ambiente de teste para avaliações subjetivas DCR e ACR .........................................33

4.1 Resultados das métricas PSNR, SSIM e VQM obtidos da comparação entre as sequências de vídeo original e degradado

4.2 Gráfico da métrica PSNR (dB) por variação de atraso (ms) com valores entre 0 e $20 \mathrm{~ms}$ e atraso médio de $100 \mathrm{~ms}$ para os vídeos Akiyo, Foreman e Football..

4.3 Gráfico da métrica SSIM por variação de atraso (ms) com valores entre 0 e $20 \mathrm{~ms}$ e atraso médio de 100 ms para os vídeos Akiyo, Foreman e Football.

4.4 Gráfico da métrica VQM por variação de atraso (ms) com valores entre 0 e $20 \mathrm{~ms}$ e atraso médio de 100 ms para os vídeos Akiyo, Foreman e Football. 
4.5 Gráfico da métrica PSNR (dB) por variação de atraso (ms) com valores entre 8 e $12 \mathrm{~ms}$ e atraso médio de $100 \mathrm{~ms}$ para os vídeos Akiyo, Foreman e Football.

4.6 Gráfico da métrica SSIM por variação de atraso (ms) com valores entre 8 e 12 ms e atraso médio de 100 ms para os vídeos Akiyo, Foreman e Football.

4.7 Gráfico da métrica VQM por variação de atraso (ms) com valores entre 8 e 12 ms e atraso médio de $100 \mathrm{~ms}$ para os vídeos Akiyo, Foreman e Football.

4.8 Gráfico da métrica ACR com intervalos de confiança de 95\% para variações de atraso com valores entre 0 e $20 \mathrm{~ms}$ e atraso médio de 100 ms para o vídeo Akiyo.....

4.9 Gráfico da métrica ACR com intervalos de confiança de 95\% para variações de atraso com valores entre 0 e 20 ms e atraso médio de 100 ms para o vídeo Foreman

4.10 Gráfico da métrica ACR com intervalos de confiança de $95 \%$ para variações de atraso com valores entre 0 e 20 ms e atraso médio de 100 ms para o vídeo Football. . .45

4.11 Gráfico da métrica DCR com intervalos de confiança de $95 \%$ para variações de atraso com valores entre 0 e $20 \mathrm{~ms}$ e atraso médio de $100 \mathrm{~ms}$ para o vídeo Akiyo.

4.12 Gráfico da métrica DCR com intervalos de confiança de $95 \%$ para variações de atraso com valores entre 0 e 20 ms e atraso médio de 100 ms para o vídeo Foreman

4.13 Gráfico da métrica DCR com intervalos de confiança de $95 \%$ para variações de atraso com valores entre 0 e 20 ms e atraso médio de 100 ms para o vídeo Football.

4.14 Gráfico da métrica ACR por variação de atraso (ms) com valores entre 0 e $20 \mathrm{~ms}$ e atraso médio de 100 ms para os vídeos Akiyo, Foreman e Football. .45

4.15 Gráfico da métrica DCR por variação de atraso (ms) com valores entre 0 e $20 \mathrm{~ms}$ e atraso médio de 100 ms para os vídeos Akiyo, Foreman e Football. 46

A.1 Secção transversal do olho humano. . .53

A.2 Função de Sensibilidade ao Contraste (CSF) normalizada. 46

A.3 Curva de transferência característica para $L^{*}$ em função de $Y$. 46

A.4 Sensibilidade espectral dos cones (S,M,L) e do bastonete. 57 


\section{Lista de Tabelas}

3.1 Características dos vídeos originais de Akiyo, Foreman e Football............................29

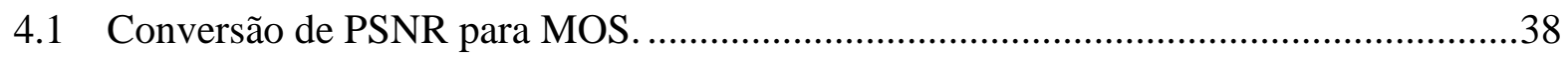

4.2 Escala de Classificação para a métrica ACR ..............................................................40

4.3 Escala de Classificação para a métrica DCR ...............................................................40

4.4 Desempenho das métricas objetivas em relação à métrica subjetiva ACR . ..................47

4.5 Desempenho das métricas objetivas em relação à métrica subjetiva DCR. .................47

4.6 Comparação entre as médias das métricas ACR e DCR com $\alpha=0,05$........................49

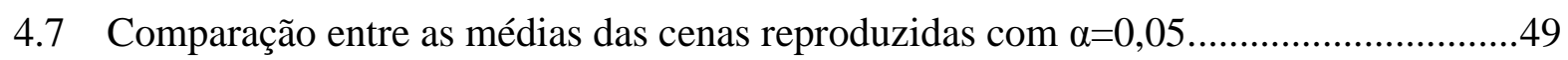

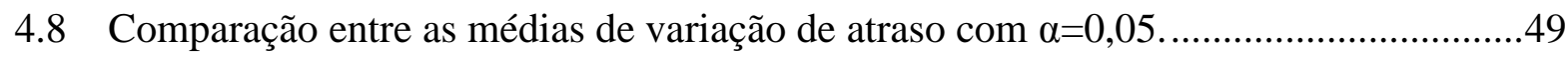




\section{Lista de Abreviaturas}

A seguir são listadas as principais abreviaturas usadas na tese.

ACR

ACR-HR

AFF

ANOVA

ATM

AVC

AVI

AVS

BRAS

CIE

CIF

CNAME

CRTP

CSF

DCR

DCT

DP

DSCQS

DSIS

DSL

DSLAM

DVMRP

DVI

FLV
Absolute Category Rating

Absolute Category Rating with Hidden Reference

Adaptive Frame-Field

Analysis of Variance

Asynchronous Transfer Mode

Advanced Video Coding

Audio Video Interleaved

Audio Video Standard

Broadband Remote Access Server

Commission Internationale de l'éclairage

Common Intermediate Format

Canonical Name

Compressed Real Time Protocol

Contrast Sensitivity Function

Degradation Category Rating

Discrete Cosine Transform

Display Port

Double Stimulus Continuous Quality Scale

Double Stimulus Impairment Scale

Digital Subscriber Line

Digital Subscriber Line Access Multiplexer

Distance Vector Multicast Routing Protocol

Digital Visual Interface

Flash Video 


\begin{tabular}{|c|c|}
\hline FR & Full Reference \\
\hline FTP & File Transfer Protocol \\
\hline GOP & Group of Pictures \\
\hline HDTV & High Definition Television \\
\hline HTTP & Hypertext Transfer Protocol \\
\hline HVS & Human Visual System \\
\hline IGMP & Internet Group Management Protocol \\
\hline IP & Internet Protocol \\
\hline IEC & International Electrotechnical Commission \\
\hline IETF & Internet Engineering Task Force \\
\hline IPS & In Plane Switching \\
\hline ISO & International Organization for Standardization \\
\hline ITU & International Telecommunication Union \\
\hline JND & Just-Noticeable Differences \\
\hline JVT & Joint Video Team \\
\hline $\mathbf{L A N}$ & Local Area Network \\
\hline LC & Local Constrast \\
\hline LCD & Liquid Crystal Display \\
\hline MAFF & Macroblock Adaptive Frame-Field \\
\hline ME & Motion Estimation \\
\hline MOS & Mean Opinion Score \\
\hline MOSPF & Multicast Open Shortest Path First Protocol \\
\hline MPEG & Moving Picture Experts Group \\
\hline MSE & Mean Squared Error \\
\hline MSU & Moscow State University \\
\hline MTS & MPEG Transport Stream \\
\hline MTU & Maximum Transmission Unit \\
\hline NETEM & Network Emulator \\
\hline NR & No Reference \\
\hline OSI & Open systems Interconnection \\
\hline $\mathbf{P} 2 \mathbf{P}$ & Peer-to-peer \\
\hline PAFF & Picture Adaptive Frame-Field \\
\hline $\mathbf{P C}$ & Pair Comparison \\
\hline PDT & Packet Delay Transfer \\
\hline
\end{tabular}




\begin{tabular}{|c|c|}
\hline PDV & Packet Delay Variation \\
\hline PES & Packetized Elementary Streams \\
\hline PIM & Protocol Independent Multicast \\
\hline PLR & Packet Loss Rate \\
\hline PSNR & Peak Signal to Noise Ratio \\
\hline PSTN & Public Switched Telephone Network \\
\hline PT & Payload Type \\
\hline QoS & Quality of Service \\
\hline RFC & Request for Comments \\
\hline RLE & Run-Length Encoding \\
\hline $\mathbf{R} \mathbf{R}$ & Reduced Reference \\
\hline RTCP & Real Time Transport Control Protocol \\
\hline RTP & Real Time Transport Protocol \\
\hline SCSF & Spatial Contrast Sensitivity Function \\
\hline SDSCE & Simultaneous Double Stimulus for Continuous Evaluation \\
\hline SSCQE & Single Stimulus Continuous Quality Scale \\
\hline SSIM & Structural Similarity Index \\
\hline TCP & Transmission Control Protocol \\
\hline UDP & User Datagram Protocol \\
\hline VCEG & Video Coding Experts Group \\
\hline VLAN & Virtual Local Area Network \\
\hline VLC & VideoLan Client \\
\hline VQEG & Video Quality Expert Group \\
\hline VQM & Video Quality Measure \\
\hline VQMT & Video Quality Measurement Tool \\
\hline WMV & Windows Media Video \\
\hline
\end{tabular}




\section{Capítulo 1}

\section{Introdução}

Neste capítulo daremos destaque para os tópicos que mostram a importância deste trabalho, juntamente com a organização estrutural da presente dissertação.

\subsection{Contexto}

A área de transporte de serviços de vídeo está em constante evolução e cresce rapidamente. Atualmente os sinais de vídeo são transportados através de diferentes tecnologias, como de rádio, cabo coaxial, micro-ondas, satélite e fibra ótica. Adicionalmente, a tecnologia com protocolo de Internet (IP - Internet Protocol) é de suma importância porque permite transportar diferentes tipos de formatos de vídeo, áudio e dados. A transição para vídeo digital, os avanços em compressão de vídeo e o crescimento da capacidade em redes IP possibilitam a entrega de conteúdo de vídeo em redes IP, para um grande número de telespectadores. Através da utilização de transporte de vídeo em redes IP obtemos benefícios como a escalabilidade, integrabilidade em uma única tecnologia para a rede de transporte, qualidade de vídeo (a qual é dependente das condições da rede), e, principalmente, a otimização dos recursos de transmissão associada à redução de custos. Em alguns casos os serviços de telecomunicações que transmitem voz, vídeo e dados através de complexas redes de transmissão, não tem uma qualidade de vídeo aceitável pelo usuário final. Por tal motivo, há a necessidade de se conhecerem os efeitos dos fatores de degradação de uma rede IP na qualidade de uma comunicação. De forma geral, são considerados diversos fatores de degradação de rede IP [COAQUIRA, ZEGARRA, \& ARJONA, 2011] como: perda de 
pacotes, atraso fixo, variação de atrasos, corrupção de pacotes, duplicação de pacotes e reordenação de pacotes.

Para avaliar a qualidade de vídeo existem dois métodos: o subjetivo, no qual o usuário outorga uma pontuação a cada vídeo assistido e o objetivo, para o qual são utilizadas as métricas baseadas em algoritmos. Os métodos de avaliação serão tratados no decorrer deste trabalho. Para os métodos objetivos são usados diversos algoritmos amplamente utilizados na avaliação de qualidade de vídeo, sendo alguns deles baseados em parâmetros estatísticos e matemáticos de imagem, como MSE (Mean Squared Error), PSNR. Outros algoritmos são baseados em características do sistema visual humano (HVS - Human Visual System), tais como as métricas SSIM [WANG, BOVIK, \& SHEIKH, 2004] e VQM [WOLF \& PINSON, 2004]. Os testes subjetivos são muito importantes, pois através dos resultados obtidos os produtos avaliados são melhorados [PARK \& HAR, 2011], obtendo-se uma maior satisfação do usuário final. Nas aplicações de vídeo, como são: IPTV, streaming de vídeo, vídeo conferencia, entre outros, as avaliações dos usuários possibilitam a detecção de erros existentes, por exemplo, os introduzidos ao longo da transmissão. Assim, os fatores de degradação de rede, podem ser estudados isoladamente, conseguindo-se obter uma relação entre o grau de satisfação do usuário final e diferentes valores do fator de degradação de rede avaliado.

A variação de atraso é um dos fatores de degradação na rede IP que tem maior impacto na qualidade do serviço de streaming de vídeo [ICKIN, DE VOGELEER, FIEDLER, \& ERMAN, 2010]. Este trabalho apresenta um estudo sobre o efeito que os diferentes valores de variação de atraso têm sobre a qualidade do vídeo transmitido ao usuário final. A avaliação de qualidade de vídeo é realizada utilizando métricas objetivas e subjetivas. $\mathrm{O}$ padrão de codificação de vídeo aqui utilizado é o H.264, devido à melhor eficiência na compressão de vídeo e maior flexibilidade na compressão, transmissão e armazenamento de vídeo [RICHARDSON, 2003]. É usado em videoconferência, transmissão de televisão e armazenamento de dados, e, em geral, reduz a taxa de bits de duas a três vezes mais do que os padrões de codificação de vídeo anteriormente propostos.

Neste trabalho é apresentado um cenário de emulação de rede isolado no qual é implementado o fator de degradação de rede (variação de atraso), originando vídeos de diferentes qualidades, percebidos pelo usuário que utiliza um serviço de streaming de vídeo. 
É importante destacar que o ambiente experimental de testes utilizado pode ser reproduzido facilmente por ser composto de ferramentas com código fonte aberto (open source).

\subsection{Objetivo}

Neste trabalho foram realizadas avaliações objetivas e subjetivas das sequências de vídeo codificadas para diferentes valores de variação de atraso, sendo utilizado um cenário de rede totalmente isolado. Assim, foi possível conhecer a qualidade de vídeo percebida por usuários finais para diferentes níveis de variação de atraso, com a finalidade de escolher um intervalo de valores dentro do qual a qualidade do vídeo é aceitável e, sobretudo, identificar um limiar em que esta qualidade começa a gerar um nível de insatisfação para tais usuários. No caso das avaliações em que são considerados usuários, foram empregadas as métricas subjetivas ACR e DCR e feita a comparação entre si. Para o caso das métricas objetivas foram utilizadas as métricas PSNR, SSIM e VQM, que foram comparadas com as métricas subjetivas usadas neste trabalho. Uma contribuição adicional é que com a realização dos testes pode-se obter uma base de dados de vídeos degradados para diferentes valores de variação de atraso.

\subsection{Organização do Trabalho}

Este capítulo mostra a parte introdutória, apresentando uma visão geral deste trabalho, a importância e o contexto em que ele está inserido, destacando o seu principal objetivo.

No Capítulo 2 apresenta-se uma breve revisão teórica, útil para a melhor compreensão do trabalho. São discutidos o codificador de vídeo, os estimadores de qualidade de vídeo, estrutura de uma rede IP, os principais fatores de degradação de uma rede IP.

No Capítulo 3 apresenta-se o cenário experimental, em que se descreve o cenário de emulação de rede IP proposta, na qual foram gerados os vídeos degradados com variação de atraso, a informação acerca das ferramentas utilizadas, os equipamentos de teste considerados e o ambiente de teste para avaliações subjetivas. 
No Capítulo 4 apresenta-se o análise de resultados obtidos a partir da avaliação de qualidade de vídeo utilizando métricas objetivas e métricas subjetivas, e a comparação dos resultados entre as métricas objetivas e subjetivas.

No Capítulo 5 são apresentadas as conclusões relacionadas ao cenário de teste, os resultados obtidos e as propostas de trabalhos futuros. 


\section{Capítulo 2}

\section{Revisão Teórica}

Neste capítulo são abordados os tipos de codificadores de vídeo, o padrão de codificação H.264, os parâmetros de codificação, os estimadores de qualidade de vídeo, a estrutura de uma rede IP, e os principais fatores de degradação de qualidade de vídeo em uma rede de dados.

\subsection{Codificação de Vídeo}

Com o advento da tecnologia de vídeo digital, além do surgimento de uma grande quantidade de dados a serem armazenados e/ou transmitidos, houve também a necessidade da compressão de vídeo em um formato binário, de forma a reduzir o volume de dados, surgindo então os codificadores de vídeo. Os codificadores de vídeo possibilitam a compressão de sinais de vídeo [ORTEGA \& RAMCHANDRAN, 2008], em alguns casos com perda de informação. Para conseguir a compressão foi necessário desenvolver modelos de percepção do olho humano e algoritmos para descartar o máximo de informação redundante ou irrelevante com o mínimo impacto visual durante o processo de codificação, dados uma taxa fixa ou média e uma distorção máxima. A transmissão de vídeo em uma rede IP tem custos associados, sendo um dos mais importantes o uso do meio de transmissão. Portanto, procura-se transmitir a maior quantidade possível de dados, usando a técnica de compressão mais adequada e, evitando uma perda significativa da qualidade do sinal recebido. Além disso, o tipo de serviço determina a qualidade do sinal de vídeo recebido. 
Os codificadores de vídeo podem ser divididos em dois grandes grupos: Os codificadores sem perdas (lossless coders) e os codificadores com perdas (lossy coders).

- Codificadores de vídeo sem perdas: chamados de compactadores, diminuem o tamanho de um arquivo sem alterar a qualidade do vídeo, garantindo um arquivo de vídeo exatamente igual ao original no processo de decodificação. Os arquivos comprimidos resultantes são de duas a três vezes menores que o original. Os codificadores sem perda mais importante são: o codificador de Huffman e o codificador por comprimento de corrida (RLE - Run-Length Encoding).

- Codificadores de vídeo com perdas: é formado por codificadores que podem diminuir o tamanho dos arquivos de vídeo originais em até 300 vezes. $\mathrm{O}$ arquivo codificado apresenta uma perda na qualidade de vídeo, porém não é possível obter exatamente o mesmo arquivo de vídeo no processo de decodificação. A taxa de codificação tem como finalidade controlar o tamanho do arquivo de vídeo final de acordo com a qualidade de vídeo desejada. O codificador com perda mais importante é o H.264.

\subsection{Padrão de Codificação H.264}

O padrão de codificação H.264 foi obtido como resultado de um projeto conjunto entre o Video Coding Experts Group (VCEG) da International Telecommunication Union (ITU) e o Moving Picture Experts Group (MPEG) da International Organization for Standardization/International Electrotechnical Commission (ISO/IEC), a parceria dos dois grupos é conhecida por Joint Video Team (JVT). A versão da ITU do padrão de codificação de vídeo é chamado de H.264, e a versão da ISO/IEC é chamada de advanced video coding (AVC), ou formalmente chamada de ISO/IEC 14496-10-MPEG-4 part 10/AVC, por ser uma nova parte da plataforma MPEG-4.

A primeira versão do padrão H.264 foi publicada em Maio de 2003, sendo atualmente um dos formatos mais utilizados para gravação, compressão, codificação e distribuição de vídeo em alta definição. Foi desenvolvido para solucionar as deficiências dos padrões de codificação de vídeo anteriores, como o MPEG-2, o MPEG-4 part 2 e o H.263. O H.264 oferece as seguintes vantagens: 
- Melhor eficiência na compressão de vídeo;

- Melhor qualidade do sinal de vídeo;

- Maior flexibilidade na compressão, transmissão e armazenamento de vídeo;

- Compatibilidade com vídeos de alta e baixa resolução;

- Redução média de taxa de bits, de aproximadamente 50\% em relação ao MPEG-2 e o MPEG-4 part 2;

- Robustez de erro, com tolerância a erros de transmissão;

- Baixa latência, devido à qualidade de vídeo;

- Decodificação de correspondência exata, evitando erros de acúmulo.

O H.264 oferece suporte a uma ampla variedade de aplicações com requisitos diferentes em diversas taxa de bits. Para aplicações de vídeo utilizado para entretenimento. O H.264 pode fornecer um desempenho entre 1 a $10 \mathrm{Mbit} / \mathrm{s}$ com alta latência. Já para serviços de telecomunicações, tal protocolo pode fornecer um desempenho abaixo de 1Mbit/s com baixa latência. O H.264 apresenta uma ampla gama de aplicações que abrange todas as formas de vídeo digital comprimido, desde aplicações de Internet Streaming, com baixa taxa de bits, até aplicações de transmissão de sinais de televisão de alta definição (HDTV) e cinema digital com vídeo codificado quase sem perdas. Entre as principais aplicações, tem-se: videoconferência, transmissão de sinal de televisão e armazenamento de dados.

As imagens de vídeo são compostas de pixels. Os pixels são agrupados dentro de cada imagem em pequenos blocos e estes são agrupados em macroblocos. Os macroblocos podem ser combinados em slices e cada imagem pode conter muitos slices. Os slices compõem quadros, que se apresentam em diferentes tipos (Quadros Intra, Quadros Previstos e Quadros Bidirecionais). Os diferentes tipos de quadros podem ser combinados em um grupo de figuras. Para uma melhor compreensão dos parâmetros de codificação, são definidos a seguir os elementos de imagens de vídeo.

- Pixel: é o menor componente em uma imagem. O número de pixels por unidade de área é chamado de resolução;

- Blocos: são porções de uma imagem dentro de um quadro de vídeo geralmente definida por um número de pixels horizontais e verticais;

- Macroblocos: é uma região de uma imagem em uma sequência digital de imagens que pode ser utilizada para determinar a compensação de movimento a partir de um quadro de referência para outros quadros em uma sequência de imagens; 
- Slice: é uma parte de uma imagem que é utilizada em vídeo digital e é composta de um grupo contíguo de macroblocos, podendo variar de tamanho e forma;

- Quadro: é uma única imagem dentro de uma sequência de imagens que compõem o vídeo;

- Quadros Intra (I-Frames: Intra Frames): são imagens completas dentro de uma sequência de imagens, utilizadas como referência para outros quadros de imagens comprimidas, apresenta a característica de ser completamente independente de outros quadros;

- Quadros Previstos (Predicted Frames: P-Frames): são imagens dentro de uma sequência de imagens que são criadas utilizando informação de outras imagens, como as de Quadros Intra;

- Quadros Bidirecionais (Bi-Directional Frames: B-Frames): são imagens dentro de uma sequência de imagens que são criadas utilizando informação de imagens anteriores, como as de Quadros Intra e Quadros Previstos.

Os parâmetros de codificação mais importantes que podem originar um maior impacto na qualidade de vídeo codificado com o padrão H. 264 são:

- Taxa de codificação (Code Rate): representa a relação entre o tamanho de um bloco de informação de $k$ bits e o tamanho de um bloco codificado de $n$ bits, em que $n$ é maior que $k$;

- Grupo de Figuras (GOP: Group of Pictures): agrupamento de quadros que possibilitam o acesso randômico. Reduz a redundância temporária nos quadros (de quadro a quadro) do conteúdo de vídeo e consiste de diferentes quadros (Intra Frames ou I-Frames, Predicted Frames ou P-Frames, e Bi-Directional Frames ou BFrames). GOP's de tamanho menor proporcionam uma recuperação mais rápida da sequência, em caso de erros durante a decodificação. Os GOP's são compostos de 12 a 15 quadros, proporcionando uma melhor relação entre tamanho e qualidade de vídeo codificado;

- Codificação adaptativa quadro-campo (AFF: Adaptive Frame-Field): otimiza a compressão de sequências entrelaçadas de vídeo, permitindo que os macroblocos sejam codificados de forma a produzir o melhor resultado taxa-distorção. Temos dois modos de codificação adaptativa a quadro-campo ao nível de quadro (PAFF: Picture Adaptive Frame-Field) e ao nível de macrobloco (MAFF: Macroblock Adaptative 
Frame-Field). Adicionalmente, temos a codificação unicamente de quadros (Frame Only) e a codificação unicamente de campos (Field Only);

- Filtro redutor de blocagem: filtro adaptativo que reduz artefatos de bloco introduzidos pelo processo de quantização, que se tornam perceptíveis especialmente em vídeos muito comprimidos;

- Tamanho de Bloco para Estimação de Movimento (ME: Motion Estimation): a estimação de movimento é o processo de busca de uma região fixa em um quadro anterior de vídeo para encontrar o bloco correspondente de pixels do mesmo tamanho tomando em consideração o quadro atual de vídeo. O bloco de estimação de movimento é o de maior complexidade computacional do codificador e pode ser implementado de maneira paralela com a finalidade de aumentar a taxa de processamento deste bloco.

\subsection{Estimadores de Qualidade de Vídeo}

Os estimadores de qualidade de vídeo têm como objetivo dar uma pontuação a uma determinada transmissão de vídeo. Estes resultados são utilizados para realizar melhoras nas redes de transmissão, em geral, todos os equipamentos intervêm neste processo. As estimativas são obtidas através de métricas objetivas e subjetivas, as quais são utilizadas na avaliação da qualidade de vídeo em diferentes cenários de rede. As métricas objetivas são baseadas em modelos matemáticos para estimar a média das opiniões dos usuários. Essas métricas podem ser classificadas de acordo com a disponibilidade ou não do vídeo original, que é considerado livre de distorção ou de alta qualidade, e pode ser utilizado como referência na comparação com o vídeo degradado. As métricas objetivas são assim classificadas [BOVIK, WANG, \& SHEIKH, 2003]:

- Referência Completa (FR - Full Reference): quando o sinal de vídeo de referência não degradado está totalmente disponível;

- Referência Reduzida (RR - Reduced Reference): quando o sinal de vídeo original não é totalmente disponível;

- Sem Referência (NR - No Reference): quando as sequências de vídeo de referência não são acessíveis, chamada também de avaliação cega. 
O modelo de avaliação de vídeo no presente trabalho é do tipo de referência completa, porque utilizamos duas entradas (vídeo original e vídeo degradado) para obter a qualidade de vídeo observada pelo usuário (observador). Para comparar o vídeo original e o vídeo degradado as seguintes métricas objetivas foram consideradas:

- Relação Sinal-Ruído de Pico (PSNR: Peak Signal to Noise Ratio);

A PSNR é uma métrica amplamente utilizada pela comunidade científica e define a relação entre a máxima energia possível de um sinal e o ruído que afeta a representação desse sinal entre os quadros de vídeo original e degradado. A métrica PSNR é representada pela seguinte equação:

$$
P S N R=10 \log _{10} \frac{L^{2}}{\frac{1}{M N T} \sum_{m=1}^{M} \sum_{n=1}^{N} \sum_{t=1}^{T}[I(m, n, t)-\hat{I}(m, n, t)]^{2}}
$$

em que $L$ é a faixa dinâmica dos valores de pixel, $\mathrm{M}$ e $\mathrm{N}$ são respectivamente a largura e a altura das sequências de vídeo original e vídeo degradado, T é o número de quadros que contém as sequências, $I(m, n, t)$ e $\hat{I}(m, n, t)$ representam o pixel na posição $(m, n)$ do quadro $t$ para a sequência original e degradada, respectivamente. Apenas a título de exemplo, para um sinal de 8 bits/pixel, $L$ é igual a 255 , já que $L=2^{\# \text { bits } / \text { pixel }}-1$. A métrica PSNR tem uma faixa de valores que varia de 0 a 100 , sendo 100 o melhor valor possível.

\section{- Índice de Similaridade Estrutural (SSIM: Structural Similarity Index);}

O SSIM [BRUNET, VRSCAY, \& WANG, 2012] é uma métrica que compara cada quadro das sequências de vídeo original e vídeo degradado para quantificar a degradação sofrida. Baseia-se na idéia de que imagens naturais são altamente estruturadas, ou em outras palavras, seus pixels apresentam forte dependência, especialmente quando estão espacialmente próximos. As dependências carregam informações importantes sobre a estrutura dos objetos na cena. O algoritmo SSIM estima a similaridade entre o vídeo original e o vídeo degradado, comparando a luminosidade $l(x, y)$, contraste $c(x, y)$ e estrutura $s(x, y)$ do vídeo original $x$ e do vídeo degradado $y$ de tamanho $N \times N$, utilizando as seguintes equações: 


$$
\begin{aligned}
& l(x, y)=\frac{2 \mu_{x} \mu_{y}+C_{1}}{\mu_{x}^{2}+\mu_{y}^{2}+C_{1}}, \\
& c(x, y)=\frac{2 \sigma_{x} \sigma_{y}+C_{2}}{\sigma_{x}^{2}+\sigma_{y}^{2}+C_{2}}, \\
& s(x, y)=\frac{\sigma_{x y}+C_{3}}{\sigma_{x} \sigma_{y}+C_{3}},
\end{aligned}
$$

em que $\mu_{x}$ e $\mu_{y}$ é a média de $x$ e $y, \sigma_{x}^{2}$ e $\sigma_{y}^{2}$ é a variância de $x$ e $y$ respectivamente, $\sigma_{x y}$ é a covariância de $x$ e $y, C_{1}, C_{2}$ e $C_{3}$ são constantes que estabilizam a divisão com o denominador pico, evitando o surgimento de instabilidades quando o denominador tende a zero. Essas constantes são dadas por:

$$
\begin{gathered}
C_{1}=\left(K_{1} \cdot L\right)^{2}, \\
C_{2}=\left(K_{2} \cdot L\right)^{2}, \\
C_{3}=\frac{C_{2}}{2},
\end{gathered}
$$

em que $L$ é a faixa dinâmica dos valores de pixel, para um sinal de 8 bits/pixel, L é igual a 255 , porque $L=2^{\# \text { bits } / \text { pixel }}-1, K_{1} \ll 1$, e $K_{2} \ll 1$. Comumente $K_{1}=0,01$ e $K_{2}=0,03$. A métrica SSIM é representada pela seguinte equação:

$$
\operatorname{SSIM}(x, y)=[l(x, y)]^{\alpha} \cdot[c(x, y)]^{\beta} \cdot[s(x, y)]^{\gamma}
$$

em que $\alpha, \beta$ e $\gamma$ são parâmetros que definem a importância relativa das componentes de luminância, contraste e estrutura, respectivamente. Se $\alpha=\beta=\gamma=1$, a equação acima se simplifica a:

$$
\operatorname{SSIM}(x, y)=\frac{\left(2 \mu_{x} \mu_{y}+C_{1}\right)\left(2 \sigma_{x y}+C_{2}\right)}{\left(\mu_{x}^{2}+\mu_{y}^{2}+C_{1}\right)\left(\sigma_{x}^{2}+\sigma_{y}^{2}+C_{2}\right)}
$$

A métrica SSIM tem uma faixa de valores que varia de 0 a 1 , sendo 1 o melhor valor possível. 
- Medição de Qualidade de Vídeo (VQM: Video Quality Measure)

A VQM [XIAO, 2000] é uma métrica que está presente na recomendação J.144 da ITU [ITU-T Rec. J.144, 2009], e trata da avaliação objetiva de qualidade de vídeo digital. Esta métrica foi extensivamente testada pelo Video Quality Expert Group (VQEG). A métrica VQM [WOLF \& PINSON, 2004] usa o vídeo original e o degradado como entradas para avaliar a qualidade de vídeo. O algoritmo VQM pode ser dividido nas seguintes fases:

Calibração: nesta fase inicial tem-se por objetivo calibrar o vídeo em preparação para a fase de extração de características. Com esta proposta, são estimados e corrigidos, juntamente com os deslocamentos espaciais e temporais, os deslocamentos de contraste e brilho da sequência de vídeo degradada em relação à sequência de vídeo original;

- Extração de características de qualidade: nesta fase, um conjunto de características de qualidade que descreve as mudanças na percepção espacial, temporal e de crominância é extraído de sub-regiões espaço-temporais da sequência de vídeo (original e degradada). Esta fase segue os seguintes passos:

- Aplicar um filtro perceptivo, com a finalidade de realçar alguns aspectos de qualidade de vídeo. A componente de luminância $Y$ contém informações das bordas, utilizando o filtro perceptivo pode-se identificar com maior precisão: o embaçamento, o efeito de bloco e outros efeitos de borda. As componentes de cor $C_{b}$ e $C_{r}$ são úteis para identificar distorções de matiz e erros de transmissão. A diferenciação no tempo de componentes $Y$ de quadros consecutivos destaca movimentos não naturais e bruscos;

- Dividir a sequência de vídeo em sub-regiões espaço-temporais. Cada subregião espaço-temporal descreve um bloco de pixels $b(s, t)$ com dimensão descrita pelo número de pixels na horizontal, o número de linhas de quadros na vertical, e o tempo de duração da região, dado em unidades de quadros de vídeo;

- Extrair características de cada região. As características $f_{o}(s, t)$ e $f_{d}(s, t)$ do sinal original e degradado, são extraídas em blocos $b(s, t)$ utilizando funções matemáticas simples, como a média e o desvio padrão. Após a extração de características, o eixo temporal $t$ já não corresponde aos quadros individuais, 
e contém um número de amostras igual ao número de quadros na sequência de vídeo dividido pela extensão temporal dos blocos;

- Aplicar um limite de perceptibilidade. Uma função de corte pode ser aplicada para reduzir a sensibilidade do parâmetro às distorções imperceptíveis. A função de corte é representada pela seguinte forma:

$f_{\text {dip }}=\left\{\begin{array}{l}\max (f, T) \text { se } f \text { é positivo } \\ \min (f, T) \text { se } f \text { é negativo }\end{array} \quad\right.$ sendo $T$ é o limiar de corte.

Estimativa de parâmetros de qualidade: Nesta fase, um conjunto de parâmetros de qualidade $p(s, t)$ que descrevem as mudanças de percepção é calculado comparando as características extraídas da sequência de vídeo degradado $f_{d}(s, t)$ com aquelas extraídas da sequência de vídeo original $f_{o}(s, t)$. Os parâmetros $p(s, t)$ dos blocos $b(s, t)$ formam matrizes tridimensionais abrangendo o eixo temporal e duas dimensões espaciais, ou seja, horizontal e vertical;

Estimativa da medida de qualidade: Nesta fase final tem-se como objetivo calcular uma métrica de qualidade global através de uma combinação linear dos parâmetros calculados nas fases anteriores, como apresentado na Figura 2.1.

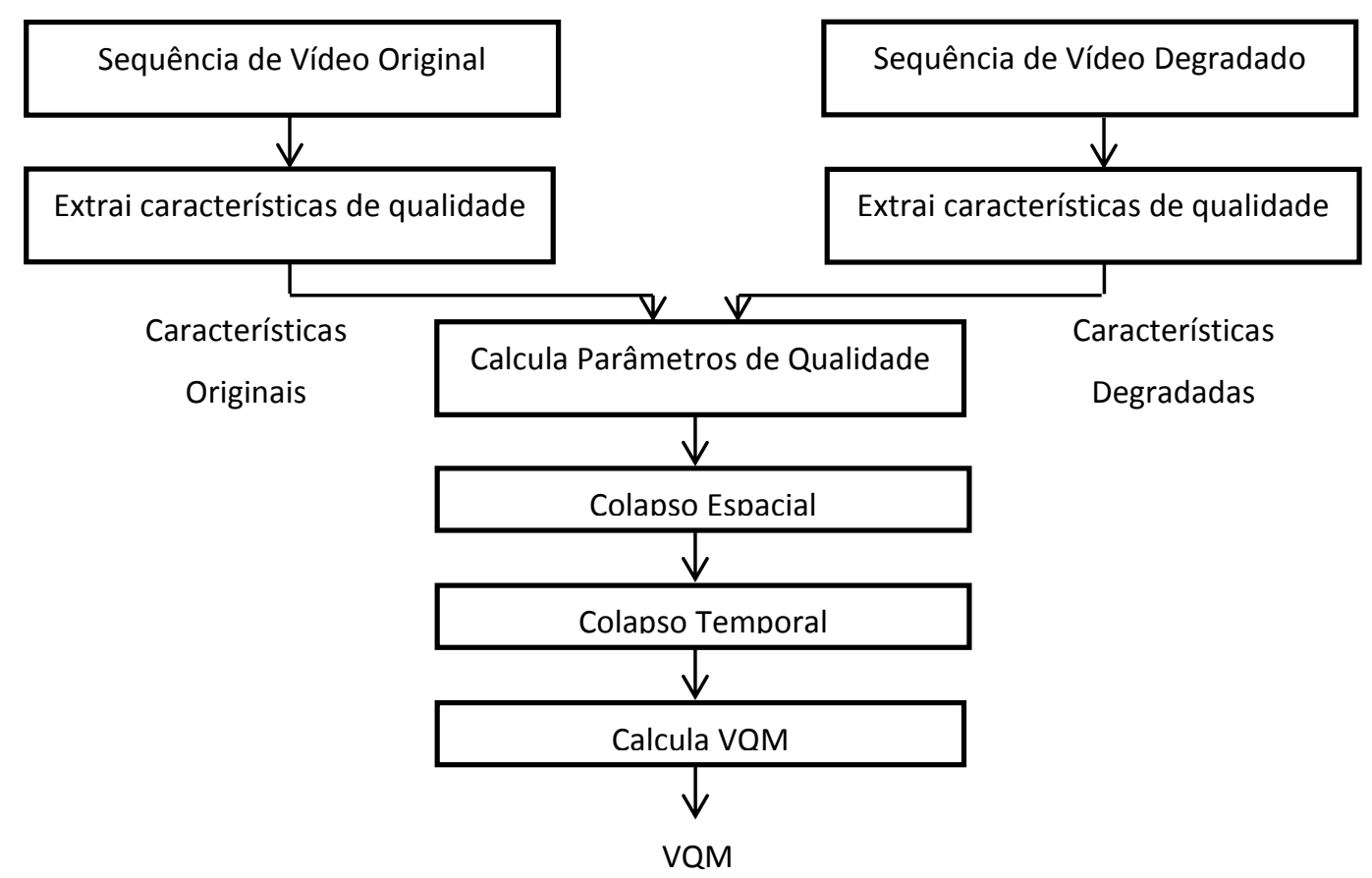

Figura 2.1: Diagrama de blocos do algoritmo da métrica objetiva VQM. 
A métrica VQM executa a transformada discreta do cosseno (DCT - Discrete Cosine Transform) para os blocos $b(s, t)$ dos quadros do vídeo original e do degradado. Os coeficientes DCT são convertidos em valores de contraste local usando os componentes DC de cada bloco (LC - Local Constrast). O modelo VQM converte os LC em valores com diferenças apénas perceptível (JND - Just-Noticeable Differences), usando funções de sensibilidade ao contraste espacial (SCSF - Spatial Contrast Sensitivity Function). Os coeficientes JND das sequências originais e degradadas são subtraídas para obter os valores de diferença Diff. Finalmente a média VQM é representada pela seguinte equação:

$$
V Q M=\text { Dist }_{\text {mean }}+0,005 \text { Dist }_{\max }
$$

em que Dist $t_{\text {mean }}$ é a média dos valores de diferença $\operatorname{Diff}$, e $\operatorname{Dist}_{\text {max }}$ é o máximo valor da diferença ponderada de todos os quadros. A métrica VQM tem valores positivos a partir de 0 , sendo 0 o melhor valor possível.

As métricas subjetivas são baseadas na percepção humana, através do julgamento de um observador. A qualidade de vídeo percebido depende da distância de visualização, do tamanho da tela, da resolução, do brilho, do contraste, da nitidez, da cor e de outros fatores. Estes métodos que foram utilizados desde os primeiros estudos referentes à avaliação de qualidade de vídeo ainda são válidos e estão descritos nas Recomendações [ITU-R Rec. BT.500-13, 2012] e [ITU-T Rec P.910, 2008]. Na Recomendação [ITU-R Rec. BT.500-13, 2012] estão definidos o número de testes e as metodologias para desenvolver os testes subjetivos de forma correta. Os métodos dessa recomendação são descritos a seguir.

- Escala de degradação com duplo estímulo (DSIS - Double Stimulus Impairment Scale);

O método DSIS é baseado na comparação de dois estímulos, fornecidos pelos sinais de vídeo original e de vídeo degradado. As condições de visualização, iluminação do ambiente, disposição das pessoas em relação ao monitor são controladas e detalhadas na recomendação. O método DSIS é o adequado para avaliar deficiências claramente visíveis, tais como artefatos causados por erros de transmissão. O teste consiste em assistir o sinal de vídeo original por uns segundos, e, posteriormente, o sinal de vídeo 
degradado pelo mesmo tempo. A mesma sequência é repetida duas vezes para cada teste. Os observadores têm que aguardar até o final de cada sequência de teste para efetuarem a avaliação. Os observadores classificam a quantidade de distorção na sequência de vídeo degradado em uma escala de cinco níveis distintos variando de "muito incômodo" a "imperceptível”. Este escala é apresentada na Figura 2.2.

- Escala de qualidade contínua de duplo estímulo (DSCQS - Double Stimulus Continuous Quality Scale);

O método DSCQS é baseado na comparação de dois estímulos, fornecidos pelos sinais de vídeo original e degradado. O método DSCQS é o adequado quando a qualidade da sequência de vídeo degradado e original são semelhantes por ser mais sensível a pequenas diferenças na qualidade de vídeo. O teste se resume em assistir a múltiplos pares de sequências que consistem de uma sequência de vídeo original e uma de vídeo degradado, que são bem curtas, com duração de 10 segundos. As duas sequências são apresentadas duas vezes alternadamente, com a ordem das duas escolhidas aleatoriamente a cada rodada. Os observadores não são informados sobre qual é a sequência de vídeo original e qual é a sequência de vídeo degradado. Os observadores classificam cada uma das duas sequências separadamente em uma escala contínua de qualidade, apresentada na Figura 2.3, a qual varia desde “péssimo" a "excelente". A análise qualitativa das avaliações baseia-se na diferença de classificação entre cada par, que é calculada a partir de uma escala numérica equivalente de 0 a 100.

- Avaliação de qualidade contínua de estímulo único (SSCQE - Single Stimulus Continuous Quality Scale);

O método SSCQE baseia-se na avaliação contínua da qualidade de vídeo sendo diferente dos anteriores, pois em vez de ver separadamente pares curtos de sequências, os observadores assistem a um programa de 20 a 30 minutos de duração que foi processado pelo sistema em teste, sendo que o vídeo pode ter ou não ter degradações. A sequência de vídeo original não é mostrada. Usando uma barra deslizante, os observadores avaliam continuamente a qualidade percebida instantaneamente em uma escala de qualidade que varia desde "péssimo" a "excelente". 
- Avaliação de qualidade contínua de duplo estímulo simultâneo (SDSCE Simultaneous Double Stimulus for Continuous Evaluation)

O método SDSCE avalia a fidelidade, comparando o sinal de vídeo original com o degradado. Pode-se avaliar o comportamento a partir de erros em velocidades de transmissão muito baixas. Os observadores assistem às sequências de vídeo original e degradado ao mesmo tempo, sendo que as duas podem ser apresentadas no mesmo monitor ou em dois monitores alinhados. Os observadores devem identificar as diferenças entre as duas sequências de vídeo e avaliar a fidelidade do sinal usando um dispositivo de voto manual. Quando a fidelidade é perfeita o cursor deve estar na parte superior da escala, no valor 100, e quando a fidelidade é nula o cursor deve estar na parte inferior da escala, no valor 0 .

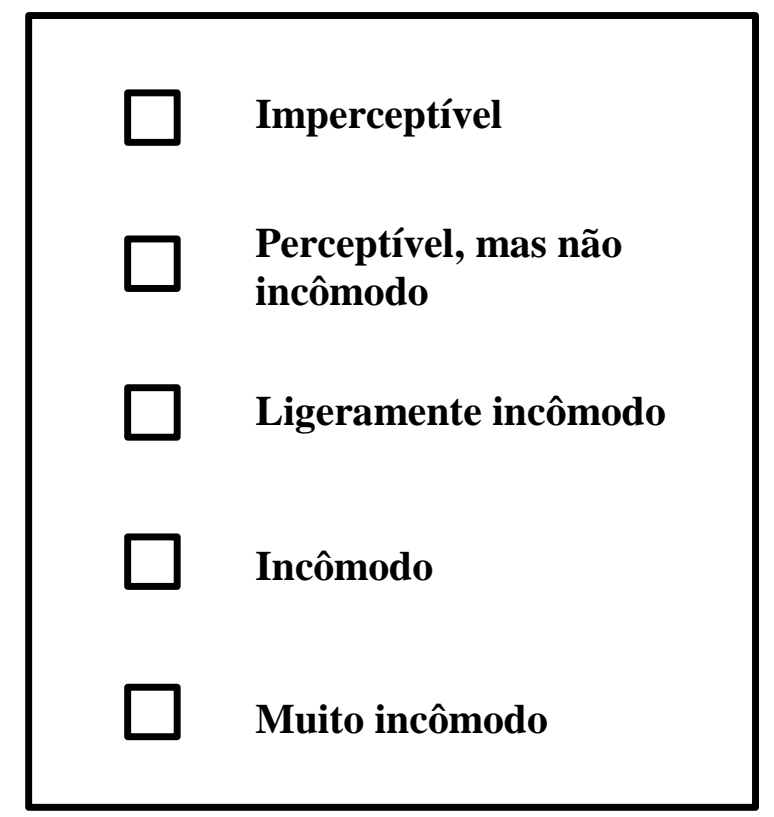

Figura 2.2: Escala de classificação para a métrica subjetiva DSIS.

Na Recomendação ITU-T P.910 descrevem-se os métodos de avaliação subjetiva de vídeo em aplicações multimídia e são apresentados a seguir.

- Índices por Categorias Absolutas (ACR - Absolute Category Rating);

O método ACR é de estímulo único para o qual as sequências de vídeo degradado são assistidas uma de cada vez, sem a sequência de vídeo original. Os observadores atribuem uma classificação para a sua qualidade global utilizando uma escala de cinco níveis distintos de "péssimo" a "excelente". O fato de as sequências de vídeo original não serem mostradas com as sequências de vídeo degradado, faz com que 
seja mais rápido em comparação com outros métodos que levam de duas a quatro vezes mais tempo.

- Índices por Categorias Absolutas com referência oculta (ACR-HR - Absolute Category Rating with Hidden Reference);

O método ACR-HR apresenta as sequências de vídeo degradado uma de cada vez. No procedimento deve-se incluir uma versão de sequência de vídeo original para cada sequência de vídeo degradado mostrado como qualquer outro estímulo de sequência de vídeo degradado. Esta inclusão é chamada de condição de referência oculta. Os observadores atribuem uma classificação para a sua qualidade global, utilizando uma escala de cinco níveis distintos de "péssimo" a "excelente".

- Índice por Categorias de Degradação (DCR - Degradation Category Rating); O método DCR é de duplo estímulo, no qual as sequências são apresentadas aos pares, sendo o primeiro estímulo a sequência de vídeo original, e o segundo a sequência de vídeo degradado. As duas sequências podem ser apresentadas de forma serial, uma sequência seguida da outra, ou pode-se apresentar de forma conjunta, no mesmo monitor. Os observadores classificam cada uma das duas sequências separadamente em uma escala contínua de qualidade que varia desde "muito incômodo" a "imperceptível".

\section{- Comparação por Pares (PC - Pair Comparison)}

O método PC é utilizado para comparar degradações produzidas por dois sistemas diferentes, sobre o mesmo sinal de vídeo original. Apresenta-se um sinal depois de passar por um sistema. Posteriormente é apresentado o mesmo sinal depois de passar por um segundo sistema. Os sistemas podem ser codificadores ou meios de transmissão. Finalmente, depois de assistir às duas sequências de vídeo, é feita uma avaliação sobre qual sinal apresentou menos degradações no contexto do cenário de teste.

Em [ITU-R Rec. BT.500-13, 2012] é apresentado um modelo para determinar a qualidade de uma transmissão de vídeo utilizando a recomendação ITU-T P.910, sendo apresentados alguns resultados em que se pode perceber a variação de qualidade quando a rede sofre mudanças temporais. A descrição das condições e procedimentos para produzir uma referência de vídeo é apresentada na Recomendação ITU-T P.930 [ITU-T Rec. P.930, 1996]. Neste trabalho propõe-se empregar a Recomendação ITU-T P.910, já que os testes serão realizados em um ambiente de rede IP utilizando o serviço de streaming de vídeo. 


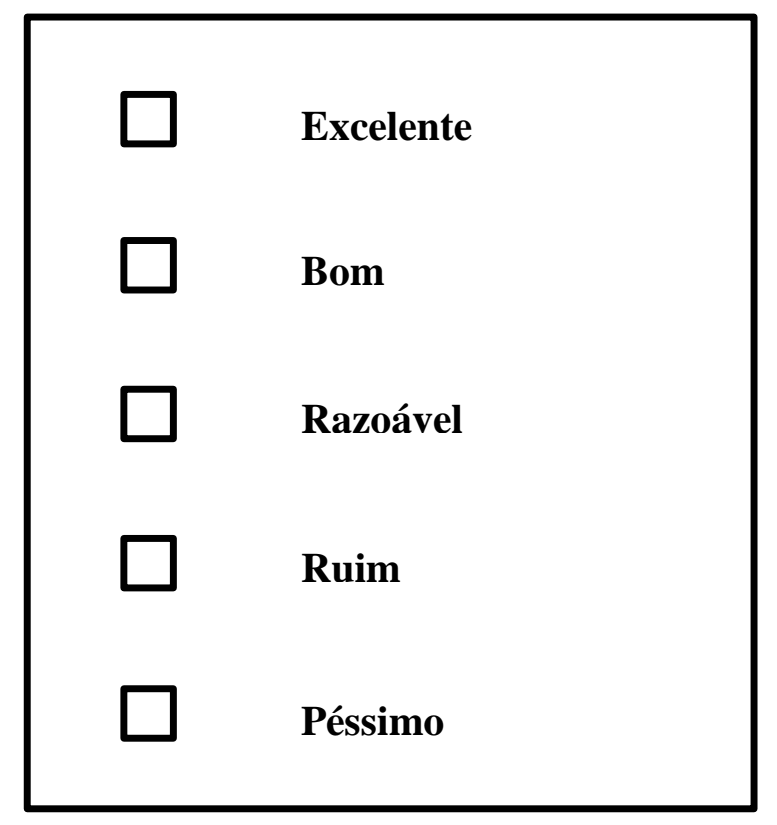

Figura 2.3: Escala de classificação para a métrica subjetiva DSCQS.

\subsection{Estrutura de uma rede IP}

As redes comutadas por pacotes podem misturar a transmissão de diversos serviços multimídia como: formatos de dados, áudio e vídeos, já que é tratada como uma transmissão de pacotes. A transmissão por pacotes apresenta vantagens na utilização adequada da largura de banda e dos recursos de rede adicionais. É muito importante levar em consideração que o controle e a gestão do transporte de vídeo requerem maiores recursos devido ao grande número de fatores que podem degradar a qualidade de transmissão de vídeo. O verdadeiro benefício do sistema de transmissão de vídeo em uma rede IP é constatado somente se a solução for baseada em uma arquitetura efetivamente distribuída. As arquiteturas para distribuição de vídeo são as seguintes:

- Cliente-servidor: Download-and-play;

A arquitetura Download-and-play, apresentada na Figura 2.4, possibilita o envio de arquivos multimídia sob demanda, através dos protocolos HTTP (Hypertext Transfer Protocol), FTP (File Transfer Protocol) e de redes Peer-to-peer (P2P) [BASET \& SCHULZRINE, 2006]. As aplicações de envio de arquivos de áudio e vídeo não requerem características especiais em relação ao atraso na rede e à variação de atraso (jitter) [ZHANG, ZHENG, \& NGEE, 2002]. A função do protocolo é controlar as 
perdas ocasionais de pacotes e efetuar as solicitações de retransmissão dos mesmos. O download de um arquivo multimídia deve ser concluído antes que o mesmo possa ser visualizado, sendo que o atraso inserido nesse processo de download varia de acordo com o tamanho do arquivo solicitado. Ao reproduzir o arquivo, o usuário tem o controle completo, podendo parar ou pausar a reprodução e retroceder ou avançar até uma determinada parte.

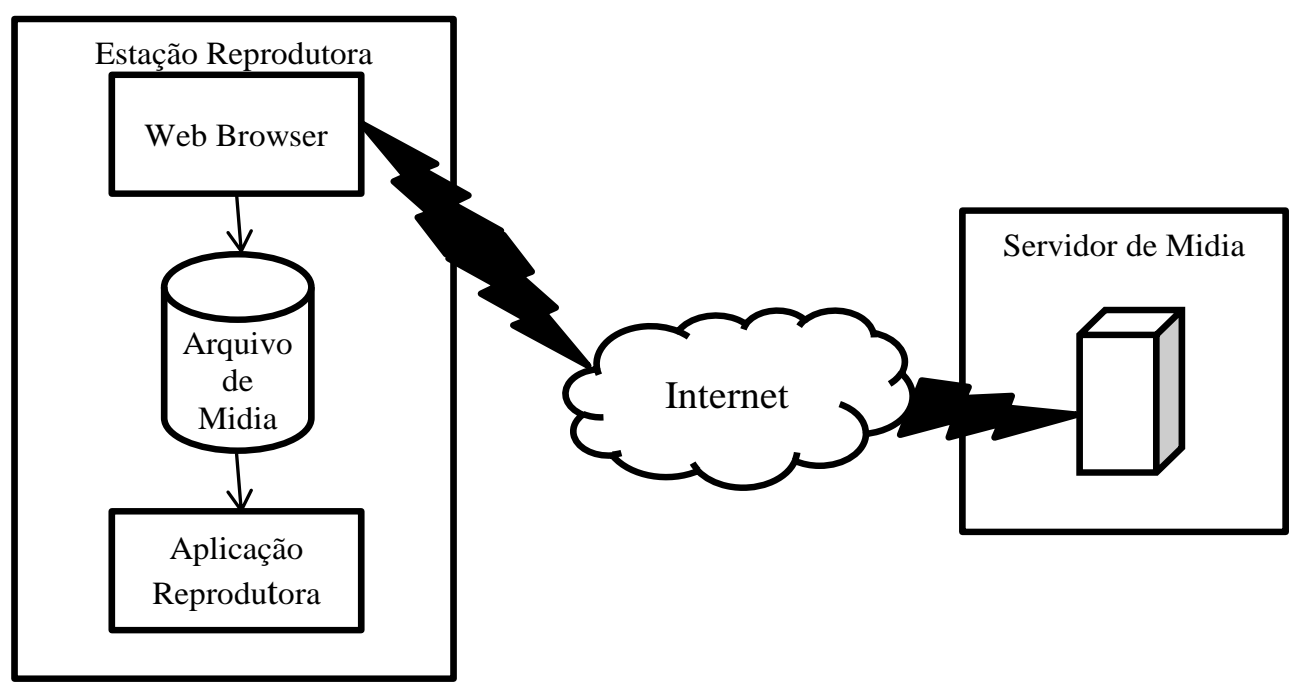

Figura 2.4: Arquitetura Cliente-servidor: Download-and-play.

\section{- Cliente-servidor: Streaming}

A arquitetura Streaming, apresentada na Figura 2.5, possibilita a apresentação do arquivo solicitado à medida que o mesmo é recebido, evitando a necessidade de efetuar o download completo do arquivo antes de iniciar a reprodução do mesmo. Para que isso seja possível, o servidor web deve enviar um meta-arquivo ao navegador (browser) do cliente, contendo informações acerca dos recursos necessários à reprodução do objeto solicitado. O navegador, após analisar o conteúdo do meta-arquivo, identifica a aplicação reprodutora associada e repassa as informações recebidas. Posteriormente, a aplicação reproduz o conteúdo solicitado.

Deve-se ressaltar que na arquitetura Streaming, as aplicações são altamente sensíveis ao atraso e à variação de atrasos, já que a mídia solicitada é decodificada e reproduzida à medida que a mesma é recebida. Um atraso na ordem de algumas 
centenas de milisegundos pode ser suficiente para que uma determinada aplicação descarte a mensagem. De qualquer forma, as aplicações multimídia são tolerantes a perdas ocasionais, sendo na maioria das vezes imperceptíveis no processo de reprodução. O usuário não tem controle completo da reprodução do arquivo, porém, não pode pular partes ou avançar até uma determinada parte. Em algumas aplicações, as funções de pausa e retrocesso podem estar habilitadas. Deve-se ressaltar que a maioria das transmissões ocorre através de vários fluxos unicast.

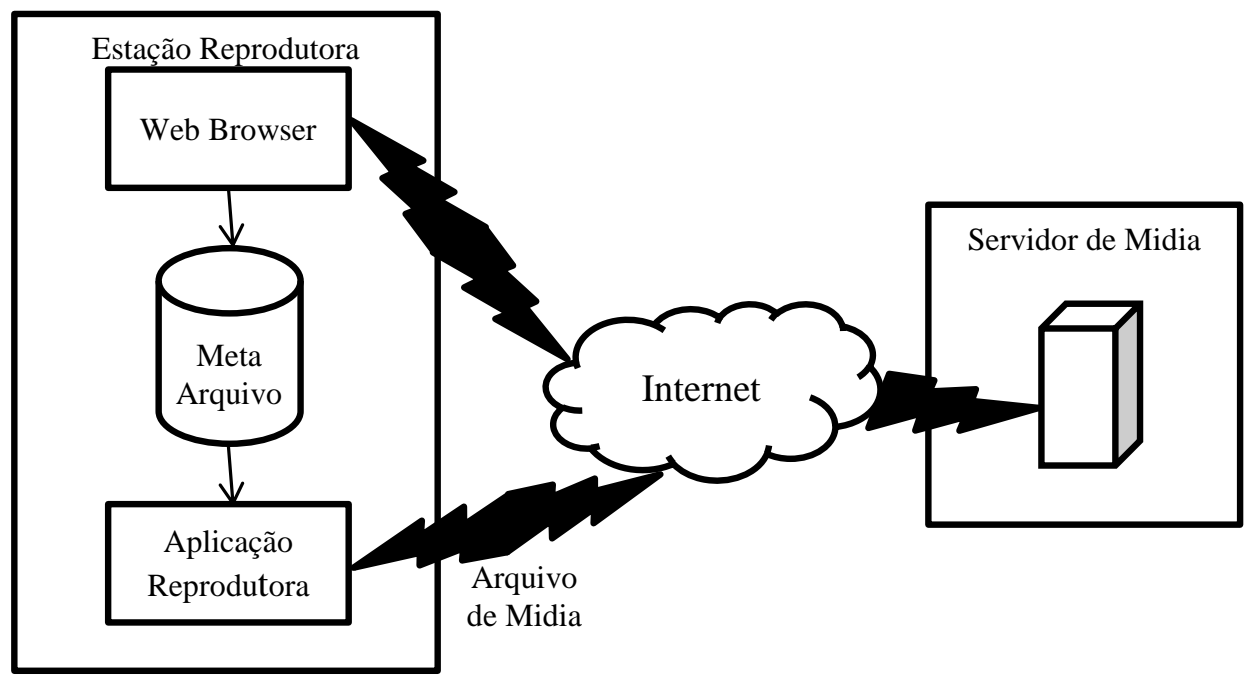

Figura 2.5: Arquitetura Cliente-servidor: Streaming.

\section{- IP Multicast}

A arquitetura IP Multicast foi proposta para melhorar a eficiência na comunicação de um usuário para múltiplos usuários e de múltiplos usuários para múltiplos usuários na Internet. Os dados só passam por um enlace de comunicação uma única vez e são replicados nos roteadores para os clientes que desejam receber o conteúdo. Deve-se ressaltar que a arquitetura IP Multicast fornece a robustez necessária para prover a escalabilidade que os usuários de serviços multimídia requerem atualmente na Internet. Os principais algoritmos de roteamento da arquitetura IP Multicast baseiamse na construção de árvores de roteamento, que podem ser compartilhadas pelo grupo ou baseadas no servidor de mídia de origem. São utilizados os protocolos DVMRP (Distance Vector Multicast Routing Protocol) [WAITZMAN, PARTRIDGE, \& DEERING, 1988], PIM (Protocol Independent Multicast) [DEERING, ESTRIN, 
FARINACCI, LIU, \& WEI, 1996], MOSPF (Multicast Open Shortest Path First Protocol) [MOY, 1994], IGMP (Internet Group Management Protocol) [CAIN, DEERING, FENNER, KOUVELAS, \& THYAGARAJAN, 2002], entre outros.

As operadoras de redes IP têm como desafio definir uma topologia para rede de acesso que possibilite a oferta de serviços e garanta os requisitos necessários para cada aplicação. Como alternativas para solucionar o desafio, surgem topologias de rede baseadas em ATM (Asynchronous Transfer Mode) e Ethernet (tecnologia de interconexão para redes locais).

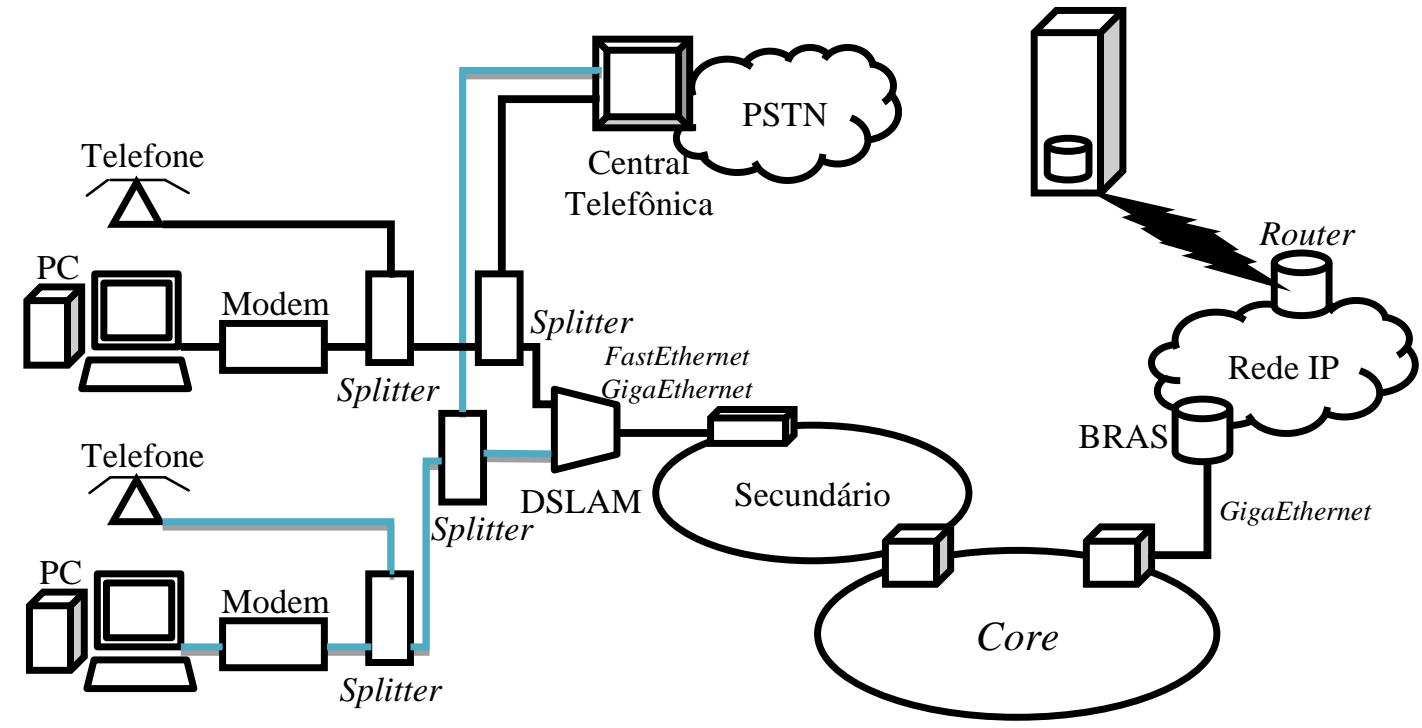

Figura 2.6: Elementos de uma rede IP.

Em uma rede IP para vídeo se distingue os seguintes elementos, apresentados na Figura 2.6:

- DSLAM (Digital Subscriber Line Access Multiplexer): é um dispositivo que recebe sinais transmitidos pelas múltiplas conexões dos assinantes do serviço DSL (Digital Subscriber Line) e os encaminha através de uma rede de alta velocidade utilizando técnicas de multiplexação. Atuando na camada de enlace, alguns DSLAM's possibilitam a classificação de tráfego em VLAN (Virtual Local Area Network), suportam requisitos de QoS (Quality of Service) e filtram pacotes;

- Splitter: é um dispositivo que possibilita a separação entre a frequência destinada à voz, através de um aparelho telefônico convencional conectado a uma PSTN (Public Switched Telephone Network), e a frequência destinada aos dados, que podem incluir 
voz e vídeo. As operadoras de telefonia fixa utilizam frequências baixas e bem definidas, entre $300 \mathrm{~Hz}$ e $4000 \mathrm{~Hz}$, facilitando o trabalho de separação realizado pelo Splitter;

- BRAS (Broadband Remote Access Server): é um dispositivo que encaminha o tráfego proveniente e destinado ao DSLAM à rede IP da operadora. As principais funções de BRAS são o gerenciamento das políticas de utilização e dos parâmetros referentes à qualidade de serviço.

\subsection{Empacotamento do sinal de vídeo}

As empresas de telecomunicações vêm se esforçando em definir um conjunto de novos mecanismos de transporte com o objetivo de transmitir voz sobre uma rede de pacotes com a mesma ou similar qualidade que tem uma rede tradicional de transporte de vídeo. Os sofisticados mecanismos de transporte devem enviar os pacotes com a maior rapidez e melhor qualidade possíveis. Os principais protocolos para transportar fluxos de áudio e vídeo MPEG-4 são os protocolos RTP (Real Time Transport Protocol) e RTCP (Real Time Transport Control Protocol). O protocolo RTP, baseado no RFC 3550 [SCHULZRINNE, CASNER, FREDERICK, \& JACOBSON, 2003] desenvolvido pela IETF (Internet Engineering Task Force), estabelece os princípios de um protocolo de transporte sobre redes que não garantem qualidade de serviço para dados em tempo real, como dados de voz e de vídeo. O protocolo RTP descreve a geração de pacotes que incluem, além dos próprios dados em tempo real a serem transmitidos, números de sequência, marcas de tempo, e monitoração de entrega. As aplicações utilizam RTP sobre protocolos de rede não confiáveis como UDP (User Datagram Protocol). Os dados obtidos de cada conjunto de amostras de voz e vídeo são encapsulados em pacotes RTP, e cada pacote RTP é encapsulado em segmentos UDP. Dentro do cabeçalho RTP é indicado com 7 bits o tipo de informação transportada (PT: Payload Type), permitindo diferenciar até 128 tipos de informação. Os valores são definidos no RFC 3551 [SCHULZRINNE \& CASNER]. O RFC 3550 estabelece também o protocolo de controle RTCP, que tem como função enviar periodicamente pacotes de controle entre os participantes de uma sessão. O protocolo RTCP tem as seguintes funções principais: 
- Prover realimentação acerca da qualidade dos dados distribuídos. Esta realimentação permite adaptar dinamicamente a codificação, ou decidir ações para solucionar problemas quando é detectada degradação na qualidade de comunicação;

- Transportar o CNAME (Canonical Name). O identificador permite associar vários fluxos RTP com a mesma origem (fluxos de áudio e vídeo provenientes do mesmo emissor);

- Adaptar dinamicamente a frequência de envio de pacotes de controle RTCP de acordo com o número de participantes da sessão.

$\mathrm{Na}$ transmissão de vídeo em uma rede IP é importante conhecer os fatores que influenciam a qualidade de serviço (QoS) e como o vídeo é transmitido na rede. Assim, as informações adicionais para uma adequada transmissão de vídeo representam uma carga adicional de bits a ser transmitida que tem influência primeiramente na taxa de transmissão, e dependendo da congestão da rede IP, na qualidade do sinal de vídeo. As sequências de vídeo (elementary streams) são empacotadas em unidades chamadas PES (Packetized Elementary Streams), que consistem de um cabeçalho de até 16 bits de dados de sequência. Os PES são empacotados em pequenos pacotes de 184 bytes, e os 4 bytes do cabeçalho totalizam 188 bytes, conformando o MTS (MPEG Transport Stream) [HARTE, 2006] que podem ser transmitidos por diferentes meios, como apresentado na Figura 2.7.

Em redes IP, o transporte de vídeo é realizado através dos protocolos RTP e RTCP. O RFC 2250 [HOFFMAN, FERNANDO, GOYAL, \& CIVANLAR, 1998] estabelece os procedimentos para transportar vídeo MPEG-1 e MPEG-2 sobre o protocolo RTP. Com o objetivo de melhorar a eficiência são transportados vários pacotes MTS de 188 bytes em um único pacote RTP. O RFC 3016 [KIKUCHI, NOMURA, FUKUNAGA, MATSUI, \& KIMATA, 2000] e o RFC 3640 [VAN DER MEER, MACKIE, SWAMINATHAN, SINGER, \& GENTRIC, 2003] estabelecem os procedimentos para transportar fluxos de áudio e vídeo MPEG-4.

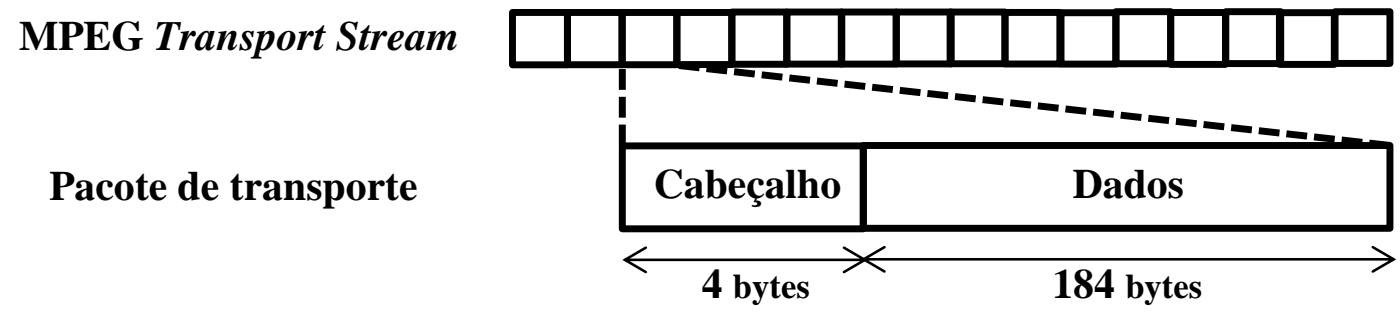

Figura 2.7: Empacotamento do sinal de vídeo. 
A Figura 2.8 apresenta os protocolos envolvidos na transmissão do vídeo codificado em uma rede IP. Nos cenários de teste implementados neste trabalho foi utilizado o protocolo de transporte UDP e os dados já codificados na camada de aplicação são empacotados utilizando o protocolo RTP, o qual pode ser visto como uma subcamada da camada de transporte [KUROSE \& ROSS, 2010]. Para o encaminhamento dos pacotes na rede é utilizado o protocolo IP, de modo que os roteadores na rede encontrem o endereço do destino. O protocolo de camada de enlace apresentado é o Ethernet, podendo ser outro protocolo, como por exemplo, o Frame-Relay (Chaveamento de Quadros).

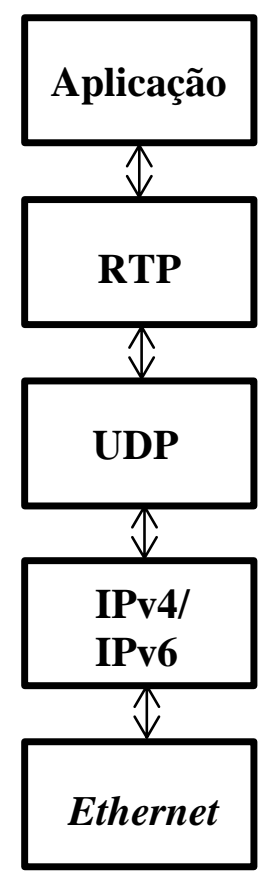

Figura 2.8: Protocolos usados na transmissão do vídeo codificado em uma rede IP.

Cada um desses protocolos insere cabeçalhos, os quais contém a informação correspondente a uma determinada camada. A informação contida nos cabeçalhos gera um overhead na informação transmitida, sendo: 20 bytes para IP, 8 bytes de UDP e 12 bytes de RTP. Para o protocolo de camada de enlace, o tamanho do cabeçalho depende do protocolo empregado, assim para Ethernet e Frame-Relay são 78 bytes e 48 bytes respectivamente. A Figura 2.9 apresenta os tamanhos, em bytes, dos diferentes cabeçalhos em uma rede Ethernet. 


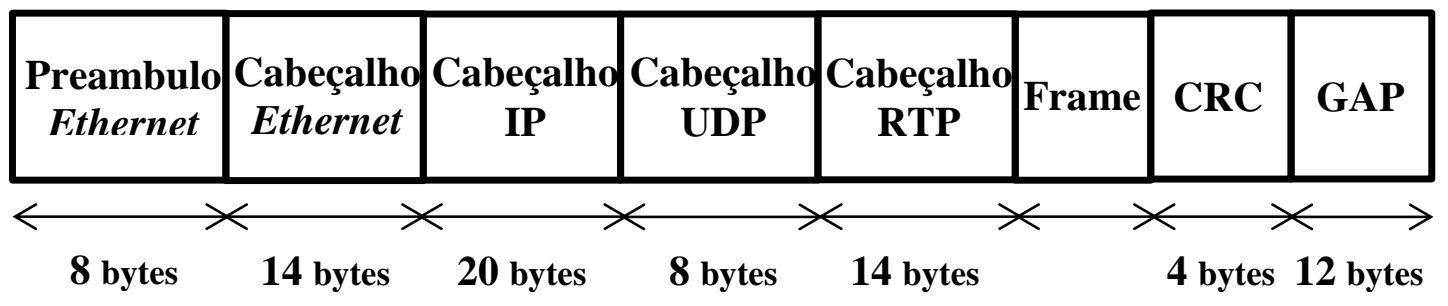

Figura 2.9: Tamanho de cabeçalhos em uma rede Ethernet.

\subsection{Fatores de degradação de rede}

Os fatores de degradação de rede podem-se dividir em:

- Taxa de perda de pacotes (PLR: Packet Loss Rate): está representada pela fração de pacotes que foram transmitidos pela rede mas não alcançam seu destino em um tempo determinado. A localização dos pacotes perdidos dentro dos dados enviados influi na qualidade de vídeo;

- Atraso ponto a ponto (PDT: Packet Delay Transfer): é definido como o tempo que demora para uma mensagem chegar desde o ponto de origem até o ponto de destino. Esse atraso ocorre devido a diversos fatores como o algoritmo de codificação, o meio de transmissão e o atraso de filas em roteadores;

- Variação de Atrasos (PDV: Packet Delay Variation): chamado de jitter, é definido em [ITU-T Rec. Y.1540, 2011] como o intervalo entre a chegada de dois pacotes consecutivos em relação ao intervalo de sua transmissão. Ele é baseado na medição do atraso fim-a-fim e é definido para pares consecutivos de pacotes. Para um melhor entendimento deste conceito é apresentada a Figura 2.10. Por outro lado, em uma rede de transmissão de pacotes é necessário programar algoritmos de controle de jitter para diminuir a variação de atrasos e consequentemente melhorar o nível de satisfação do usuário final, como os apresentados em [JAGADISH \& MANIVASAKAN, 2011] e [CHEN, LIN, \& HSIEH, 2009]. 


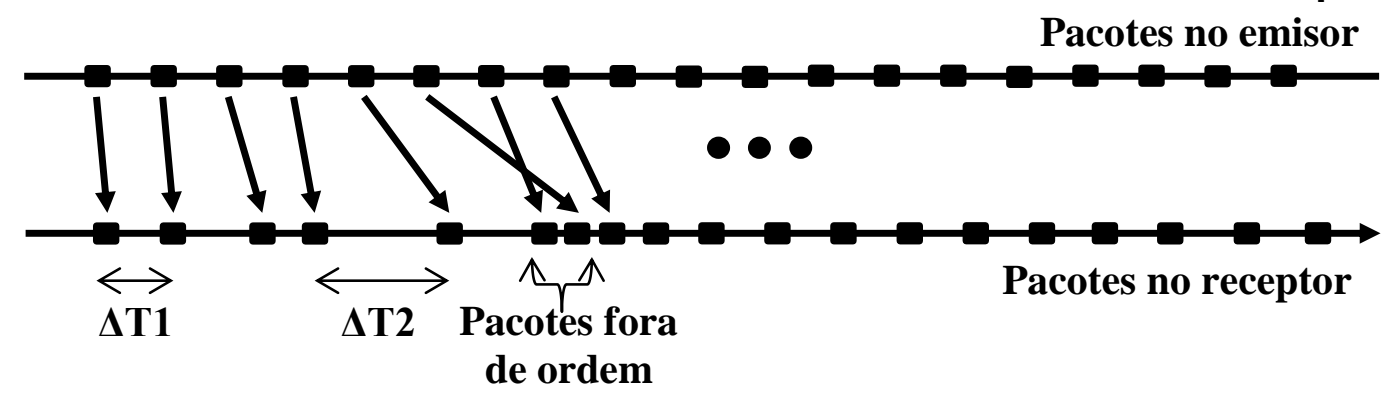

Figura 2.10: Variação de atraso de pacotes.

\subsection{Taxa de Codificação e Taxa de Transmissão}

No transporte de vídeo em uma rede IP, cada envio de uma sequência de vídeo requer uma quantidade mínima de banda para sua realização. A taxa de transmissão é calculada para o protocolo Ethernet. Adicionalmente, se descrevem alguns mecanismos para reduzir a taxa de transmissão nominal. A largura de banda necessária para transportar vídeo em uma rede IP depende dos seguintes fatores:

- Tipo de codificador;

- Período de amostragem;

- Cabeçalho RTP/UDP/IP;

- Protocolo de camada 2 do modelo OSI (Open systems Interconnection).

Para determinar a taxa de transmissão [ODOM, ICDN2, 2007] é utilizada a seguinte equação:

$$
T_{t}=\left[\frac{\left(L_{p}+C_{i p}\right)}{L_{p}}\right] T_{c}
$$

em que $L_{p}$ é o tamanho do pacote em bytes, $T_{c}$ é a taxa de codificação em bit/s, $T_{t}$ é a taxa de transmissão em bit/s e $C_{i p}$ são os cabeçalhos dos protocolos RTP/UDP/IP e dos protocolos de Camada 2: Ethernet e Frame-Relay em bytes. Os valores de $C_{i p}$ para uma rede 
Ethernet é de 78 bytes (considerando os 40 bytes dos cabeçalhos RTP/IP/UDP) e para uma rede Frame-Relay [ODOM, ICDN1, 2007] é de 48 bytes.

Para reduzir a largura de banda podem ser utilizados os seguintes mecanismos:

- Número de quadros por pacote: O codificador define o tamanho da amostra mais o número de amostras colocadas num pacote, afetando o número de pacotes enviados por segundo. Em geral, aumentando o tamanho do quadro reduz-se a banda necessária;

- Compressão de cabeçalho RTP/UDP/IP: O cabeçalho IP [CASNER \& JACOBSON, 1999] de 40 bytes é um valor constante, independentemente do codificador utilizado. Utilizando o CRTP (Compressed Real Time Protocol), os 40 bytes transformam-se em 2 ou 4 bytes, consequentemente permite uma considerável redução na taxa de transmissão. 


\section{Capítulo 3}

\section{Cenário Experimental}

Neste capítulo se descreve o cenário de emulação de rede IP para a transmissão de pacotes totalmente controlado, ou seja, sem nenhum tráfego de dado adicional que possa influenciar nas avaliações realizadas. Sobre este cenário de rede IP se efetuam testes de transmissão de vídeo sobre pacotes em comunicações ponto a ponto, em que foram gerados os vídeos degradados com diferentes valores de variação de atraso. Também descreve-se a informação acerca da base de dados de vídeos, ferramentas e equipamentos utilizados no cenário de emulação de rede e no ambiente de teste para avaliações subjetivas.

\subsection{Base de dados de vídeos}

A base de dados utilizada neste trabalho foi disponibilizada pelo [XPIH, 2010], composta por um total de 170 vídeos, sendo 108 de definição padrão e abaixo, 50 de alta definição e 12 sequências completas. Neste trabalho foram usados os vídeos Crews e News para a sessão de treino e, Akiyo, Foreman e Football para a sessão de teste. Os vídeos encontram-se no formato CIF (Common Intermediate Format) com resolução de 352x288 pixels e taxa de quadro de 29,97 quadros/segundo. Na recomendação [ITU-T Rec. P.910, 2008] o conteúdo dos vídeos é classificado por categorias. Os vídeos Akiyo, Foreman e Football mostrados na Figura 3.1 correspondem às categorias A, B e E respectivamente. Sendo que a categoria A, corresponde a uma pessoa com movimentos limitados, a categoria B coincide com uma pessoa, gráficos e maiores detalhes e, finalmente, a categoria $\mathrm{E}$ corresponde a muito movimento. Na Tabela 3.1 são especificadas as característica dos vídeos originais utilizados neste trabalho. 


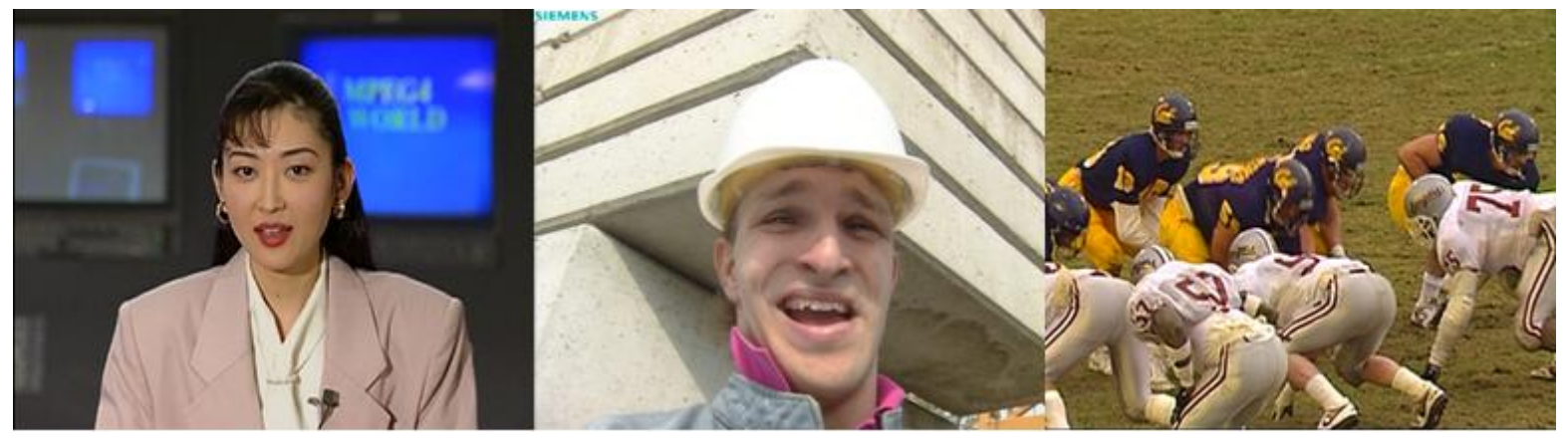

Figura 3.1: Imagens dos vídeos originais de Akiyo, Foreman e Football.

Tabela 3.1: Características dos vídeos originais de Akiyo, Foreman e Football.

\begin{tabular}{l|c|c|c|c|c}
\hline \multicolumn{1}{c|}{ Vídeo } & Resolução & \# Quadros & Quadros/s & Duração (s) & Categoria \\
\hline Akiyo & $352 \times 288$ & 300 & 29,97 & 10 & $\mathrm{~A}$ \\
Foreman & $352 \times 288$ & 300 & 29,97 & 10 & $\mathrm{~B}$ \\
Football & $352 \times 288$ & 260 & 29,97 & 8 & $\mathrm{E}$ \\
\hline
\end{tabular}

\subsection{Cenário de emulação de rede IP}

Com a finalidade de realizar os testes, se montou um cenário de rede de transmissão de pacotes totalmente controlado, ou seja, sem nenhum tráfego de dado adicional que possa influenciar as avaliações. Este cenário de teste é composto de 3 PC's, como pode ser observado na Figura 3.2, em que: PC-1 é um ponto final de comunicação que atua como um servidor Web, PC-2 é um computador que emula um canal de transmissão, sendo aqui configurados diferentes cenários de rede baseados principalmente no fator de degradação de variação de atraso e, finalmente PC-3 é um ponto final que utiliza o servidor Web para descarregar arquivos de vídeo. Na Figura 3.3 é representada a configuração lógica dos endereços IP, em que PC-2 cumpre a função de roteador da rede, interligando as redes do PC-1 e PC-3. Neste cenário é possível realizar testes considerando fatores de degradação de rede como atraso ponto a ponto, probabilidade de perda de pacotes, corrupção de pacotes, duplicação de pacotes, reordenação de pacotes e variação de atraso. É importante ressaltar que o cenário foi montado com base em softwares com código fonte aberto, sendo de fácil reprodução. 


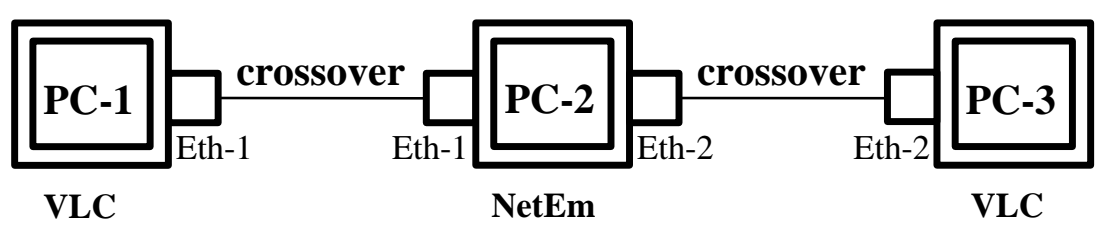

Figura 3.2: Configuração do cenário de emulação de rede IP proposto com ferramentas.

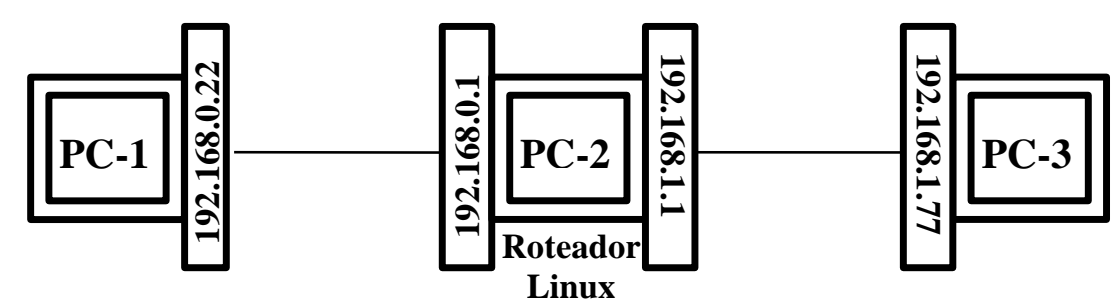

Figura 3.3: Configuração lógica de endereçamento IP para o cenário de emulação proposto.

As ferramentas utilizadas na implementação do cenário de emulação de rede, conforme a Figura 3.2 foram o NetEm (Network Emulator) [NETEM, 1991] no PC-2 e o VLC (VideoLan Client) [VLC, 2001] no PC-1 e PC-3. O NetEm é o emulador de rede usado neste trabalho, sendo possível configurar os fatores de degradação de rede, em nosso caso a variação de atraso. Encontra-se no pacote do Linux iproute2, que é uma coletânea de ferramentas para controle de pacotes TCP (Transmission Control Protocol)/IP. Para o fator de degradação de variação de atraso o NetEm utiliza uma distribuição uniforme como configuração padrão (default). Além da distribuição normal, podem ser geradas a distribuição de Pareto e a distribuição de amostra baseada em dados experimentais. $\mathrm{O}$ NetEm está limitado pelo temporizador (timer) do Linux na criação de pacotes pelo limiar de $1 \mathrm{~ms}$. A outra ferramenta utilizada no cenário proposto é o VLC, este cumpre duas funções, a primeira como administrador de servidor web, em que são alocados os arquivos de vídeos que serão visualizados pelo usuário para o cenário descrito e, a segunda função como software de gravação dos vídeos degradados no PC-3. Conforme a Figura 3.2 o servidor PC1 e o cliente PC-3 têm instalado o VLC player, os computadores são conectados em uma rede local LAN (Local Area Network) usando a tecnologia de interconexão Ethernet através de cabos de par trançado no padrão RJ-45. A configuração do VLC para transmissão e gravação de vídeos encontra-se no [DWARAKA \& KILARI, 2010]. Neste trabalho é usado o protocolo UDP para transmitir os vídeos, e a transcodificação para escolher o formato de vídeo e a taxa de bits desejada. Para tanto, foi usado o codificador H.264 e o encapsulamento MPEG-TS (MPEG transport stream) requerido para transmissão UDP. Na codificação de 
vídeo o VLC utiliza uma taxa de bits de $800 \mathrm{kbps}$ e uma taxa de quadros de $25 \mathrm{fps}$ como codificação padrão. Neste trabalho, também foi usada a configuração com taxa de bits de 1024 kbps e taxa de quadros de 29,97 fps. O tamanho dos buffers de leitura e escritura é 16384 bytes. A decodificação e a codificação são realizadas em pacotes de 188 bytes.

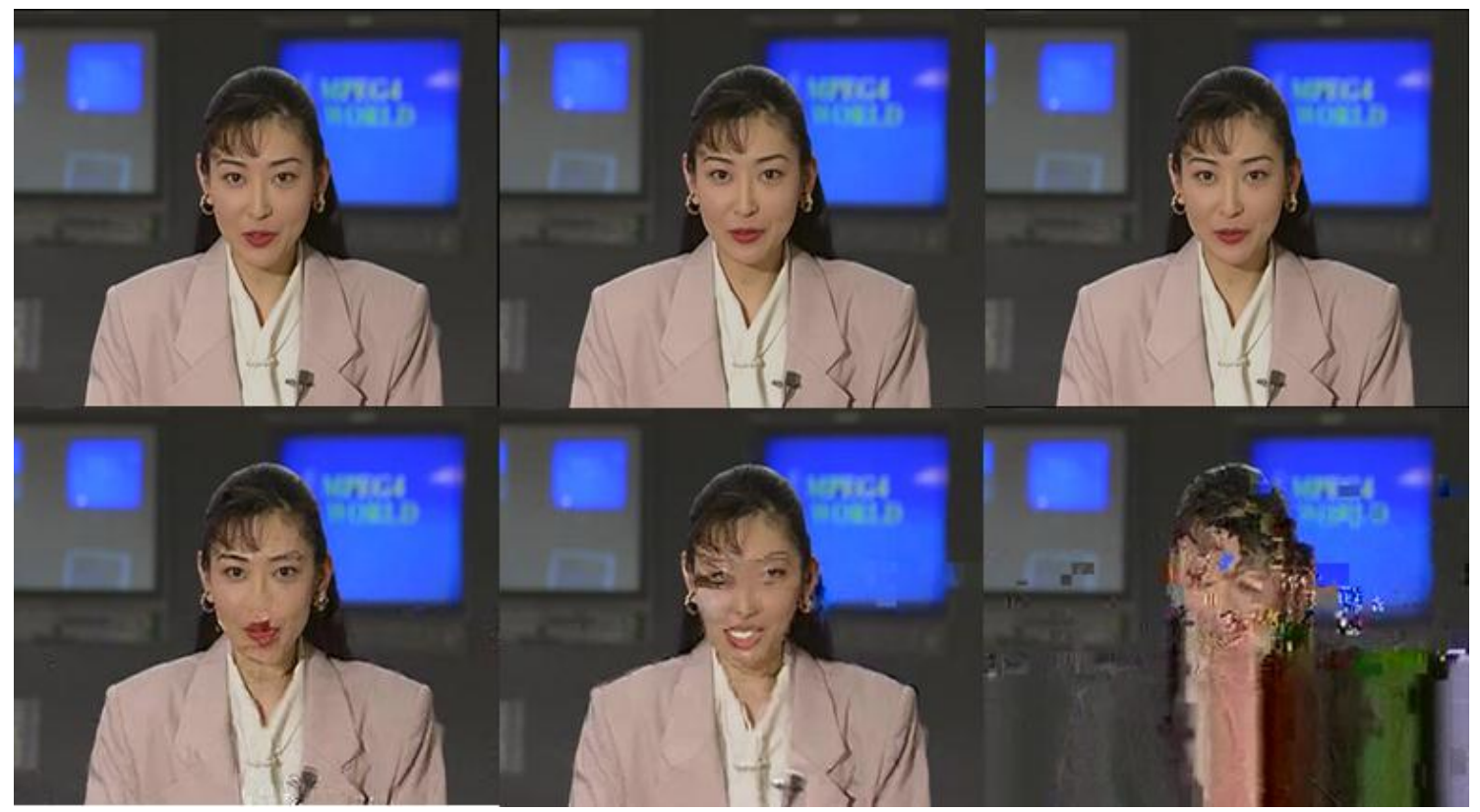

Figura 3.4: Vídeo degradado de Akiyo com variação de atraso de 0,4,8,12,16 e 20 ms.

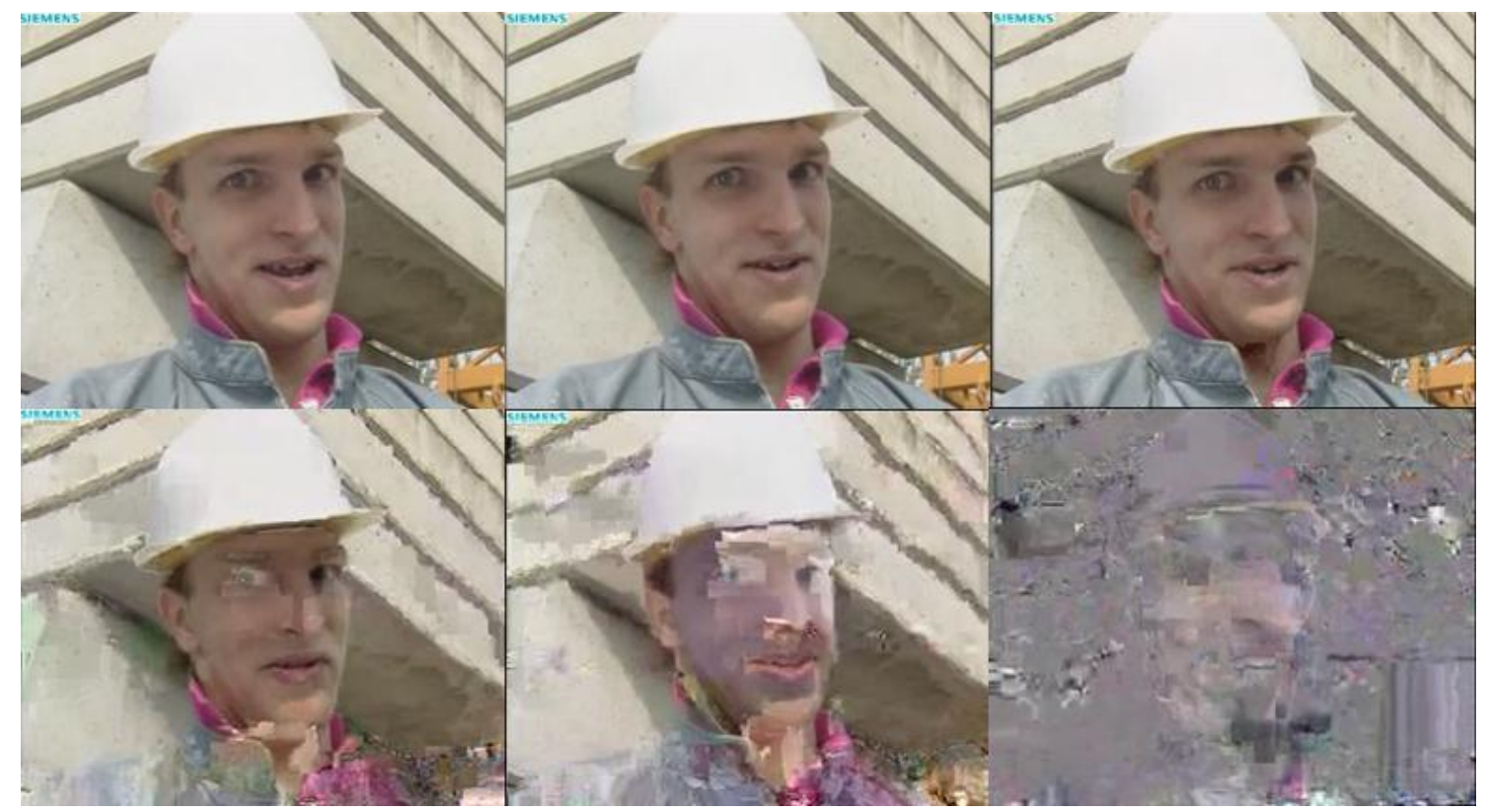

Figura 3.5: Vídeo degradado de Foreman com variação de atraso de 0,4,8,12,16 e 20 ms. 


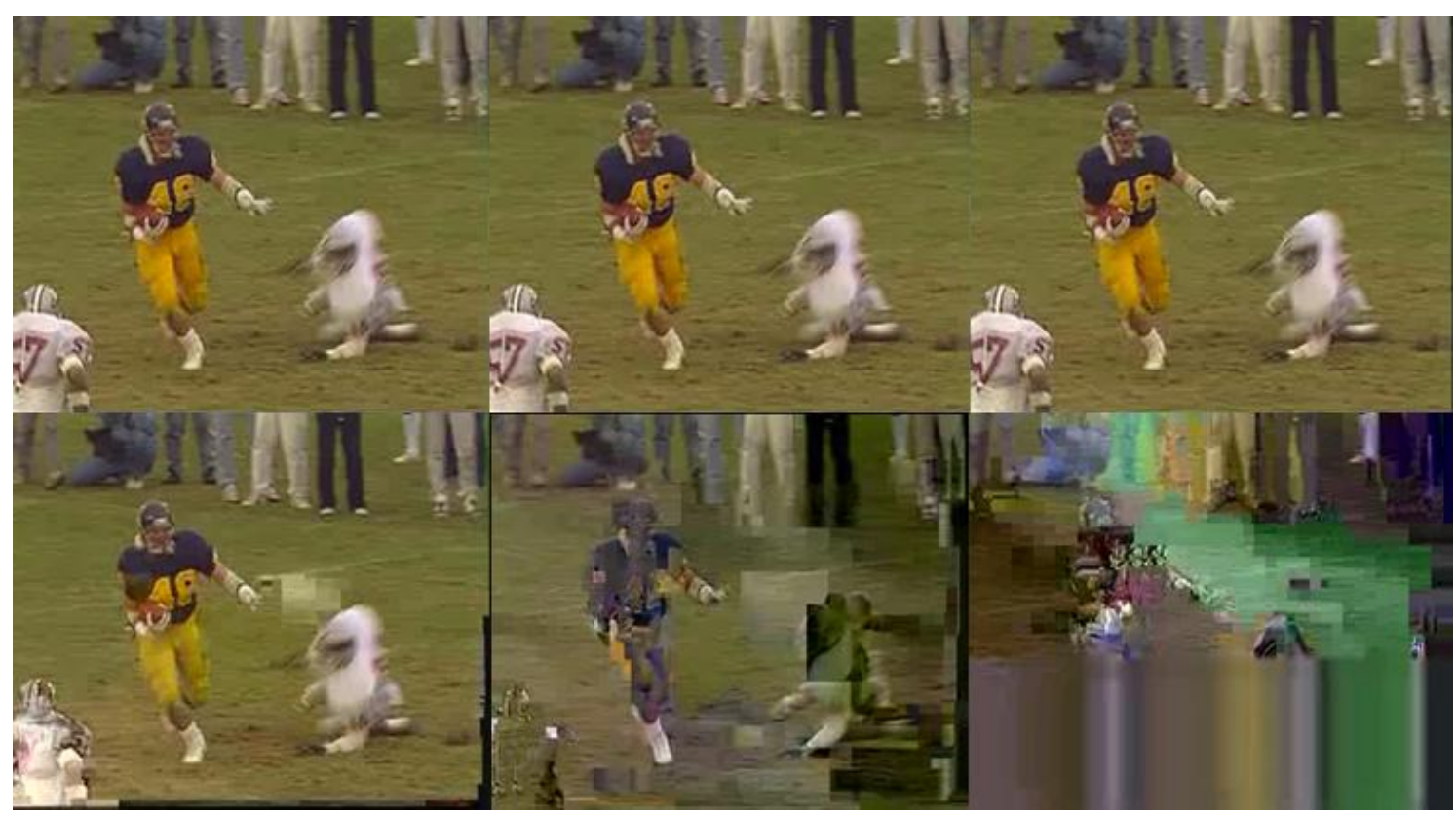

Figura 3.6: Vídeo degradado de Football com variação de atraso de 0,4,8,12,16 e 20 ms.

$\mathrm{Na}$ transmissão, a interface de rede empacota uma unidade máxima de transmissão (MTU - Maximum Transmission Unit) de 1500 bytes.

Pode-se observar nas Figuras 3.4 a 3.6 o efeito do fator de degradação de variação de atraso com valores de $0,4,8,12,16$ e $20 \mathrm{~ms}$ para o mesmo quadro das sequências Akiyo, Foreman e Football respectivamente. Para as imagens mostradas, observa-se que a qualidade dos vídeos diminui conforme o fator de degradação de variação de atraso aumenta. $\mathrm{O}$ modelo de avaliação de vídeo empregado neste trabalho é do tipo FR (referência completa), pois para a determinação da qualidade do vídeo observado pelo usuário são utilizadas duas entradas: o arquivo de vídeo de referência e o arquivo de vídeo degradado.

Na avaliação objetiva de qualidade de vídeo, foi utilizada a versão freeware do software MSU Video Quality Measurement Tool (VQMT) [MSU Graphics \& Media Lab, 2011], que é uma ferramenta para determinar a qualidade de vídeo comparando o vídeo original e o vídeo degradado, através de métricas objetivas como: PSNR, SSIM [WANG, BOVIK, \& SHEIKH, 2004] e VQM [XIAO, 2000]. Tal software suporta diferentes formatos de vídeo com entrada como: avi (Audio Video Interleaved), avs (Audio Video Standard), yuv, bmp, mov, wmv (Windows Media Video), mp4, mpeg, flv (Flash Video) e outras. Neste trabalho, foi realizada a conversão dos vídeos de referência e degradados para o formato avi, já que a ferramenta VQMT utiliza este formato como base. Na avaliação objetiva são usadas as métricas PSNR, SSIM e VQM para a componente de luminância (Y). 


\subsection{Ambiente de teste para avaliações subjetivas}

Neste trabalho os testes subjetivos são realizados utilizando os mesmos arquivos de vídeo empregados nos testes objetivos, considerando os arquivos de vídeo original e degradado de cada cenário de teste empregado. Os equipamentos de teste são utilizados principalmente nas métricas subjetivas para avaliar a qualidade de vídeo. Os testes são realizados de acordo com metodologias precisas e em um ambiente de teste controlado para produzir resultados confiáveis, evitando a influência de fatores externos [ITU-T Rec. P.910, 2008]. Um elemento importante dos equipamentos de teste são os monitores. São utilizados dois monitores LCD (Liquid Crystal Display) de alta qualidade, com pequeno tempo de resposta e tecnologia IPS (In Plane Switching), com ângulo de visão excelente e grande fidelidade das cores . Os dois monitores mostrados na Figura 3.7 são conetados a uma placa de vídeo, utilizando duas portas DVI (Digital Visual Interface). Os monitores tem um tamanho de visualização diagonal de 21.5", resolução de tela de 1920x1080 pixels, tempo de resposta cinza a cinza de $8 \mathrm{~ms}$, luminosidade de $250 \mathrm{~cd} / \mathrm{m}^{2}$, taxa de contraste dinâmico de 10000:1, painel IPS e uma taxa de proporção Widescreen 16:9.
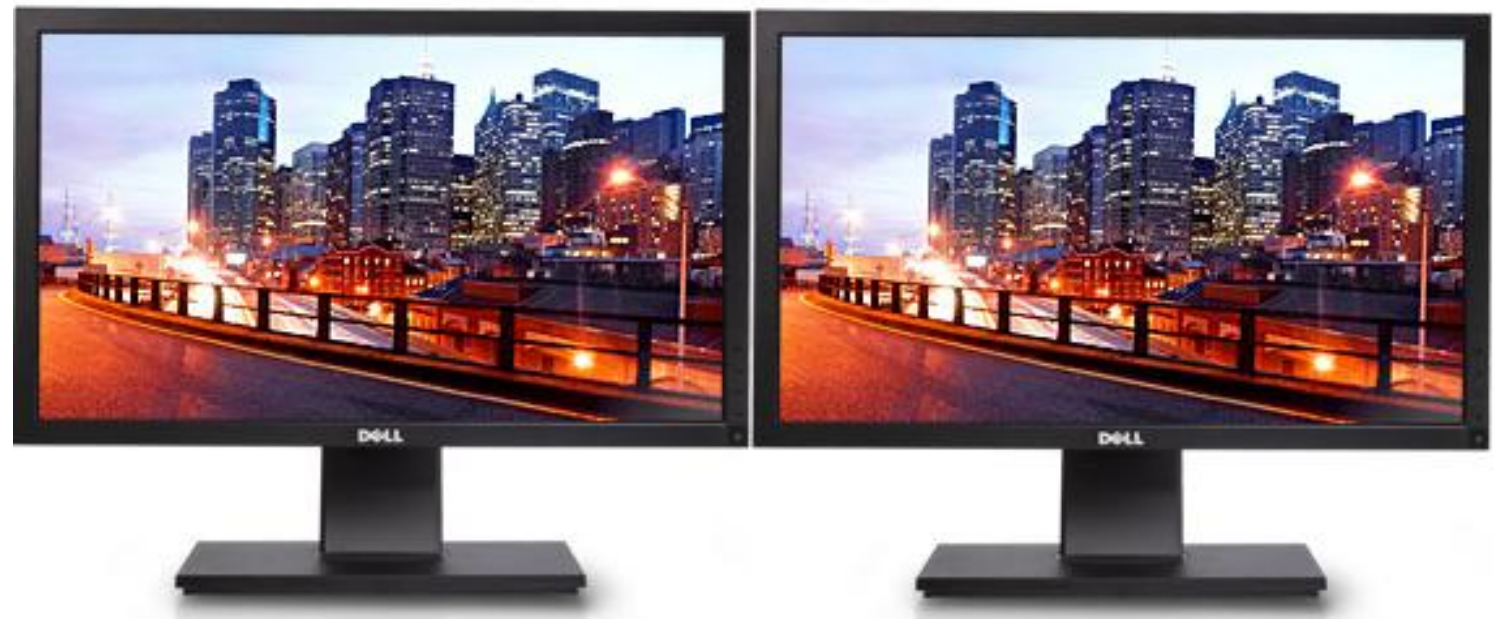

Figura 3.7: Ambiente de teste para avaliações subjetivas DCR e ACR.

A avaliação foi feita nas mesmas condições para todos os observadores. Considerou-se a distância de observação de $50 \mathrm{~cm}$, a duração do teste foi entre 20 e 25 minutos, o fundo do ambiente branco, e o fundo do monitor cinza. Após de cada reprodução de vídeo para a métrica ACR e também após a apresentação dos pares de vídeo referência e degradado para 
a métrica DCR foi realizada a avaliação pelo observador, sempre mantendo-se o fundo cinza na votação, conforme a recomendação [ITU-T Rec. P.910, 2008].

$\mathrm{Na}$ avaliação de qualidade subjetiva é utilizado o freeware software MSU Perceptual Video Quality Task Manager [MSU Graphics \& Media Lab, 2011] para gerar a ordem aleatória de reprodução dos vídeos degradados para a métrica ACR e dos pares de vídeos referência e degradado para a métrica DCR. Para o armazenamento dos índices de qualidade das avaliações subjetivas é utilizada a versão freeware do software MSU Perceptual Video Quality Player [MSU Graphics \& Media Lab, 2011]. Os índices de qualidade são armazenados em uma base de dados de forma automática, graças à modificação introduzida no referido software para permitir a avaliação através das métrica ACR e DCR, já que o mesmo não apresenta a opção de escolha entre tais métricas. É importante ressaltar que o cenário foi montado com base em softwares livres, sendo, portanto, de fácil reprodução. 


\section{Capítulo 4}

\section{Análise dos Resultados}

Neste capítulo, são mostrados resultados obtidos a partir das avaliações objetiva e subjetiva de qualidade de vídeo no cenário de rede IP proposto em função da variação de atraso. Inicialmente, são apresentados resultados usando as métricas objetivas PSNR, SSIM e VQM, para a componente de luminância (Y), e em seguida usando as métricas subjetivas ACR e DCR. Além disso, as métricas objetivas e subjetivas são comparadas entre si.

\subsection{Resultados da Avaliação Objetiva}

Nesta seção, para efeitos de avaliação do sinal de vídeo degradado em relação ao de referência, consideram-se as métricas PSNR, SSIM e VQM que contemplam apenas a componente de luminância (Y). Para comparação entre quadros iguais, os valores das médias PSNR, SSIM e VQM são iguais a 100, 1 e 0, respectivamente. No caso das métricas PSNR e SSIM valores altos são melhores, e para a métrica VQM valores baixos são melhores. A medida em que a variação de atraso é incrementada a média das métricas PSNR e SSIM diminui e a média da métrica VQM aumenta.

Na Figura 4.1 são apresentados os resultados das métricas PSNR, SSIM e VQM para a componente de luminância obtidos da comparação entre os quadros 92 das sequências de vídeo original e degradado de Akiyo. As cores para PSNR são apresentadas em ordem crescente: vermelho, amarelo, verde, azul e preto, quanto maior o valor da média PSNR, menor é a diferença entre quadros. Para SSIM e VQM as áreas com maior brilho correspondem à maior diferença entre quadros. 


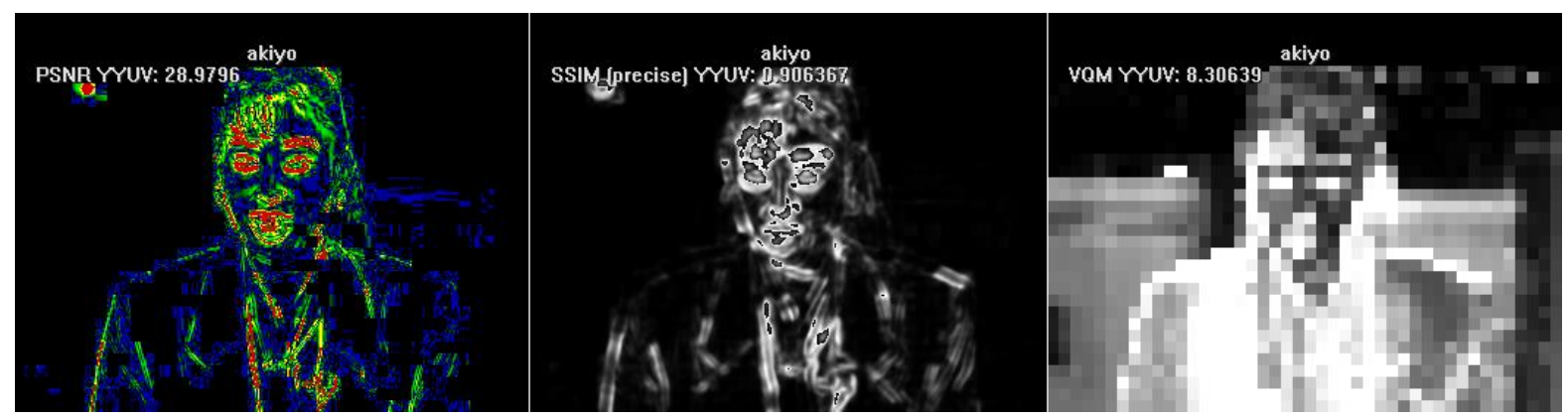

Figura 4.1: Resultados das métricas PSNR, SSIM e VQM obtidos da comparação entre as sequências de vídeo original e degradado.

As diferentes métricas objetivas apresentam médias com muitas diferenças, e quanto menor é a variação de atraso melhor é o resultado. A qualidade de vídeo é menor em função do aumento da variação de atraso, conforme os resultados mostrados nas Figuras 4.2, 4.3 e 4.4 usando PSNR, SSIM e VQM respectivamente, para os vídeos Akiyo, Foreman e Football com variação de atraso entre 0 e 20 ms e com atraso médio de 100 ms. Nas Figuras 4.5, 4.6 e 4.7 são apresentados os resultados usando PSNR, SSIM e VQM respectivamente, considerando-se os mesmos vídeos, porém com variação de atraso entre 8 e $12 \mathrm{~ms}$ e atraso médio de $100 \mathrm{~ms}$. Este intervalo com passos de $0,5 \mathrm{~ms}$ corresponde ao maior decrescimento da qualidade de vídeo para o cenário proposto.

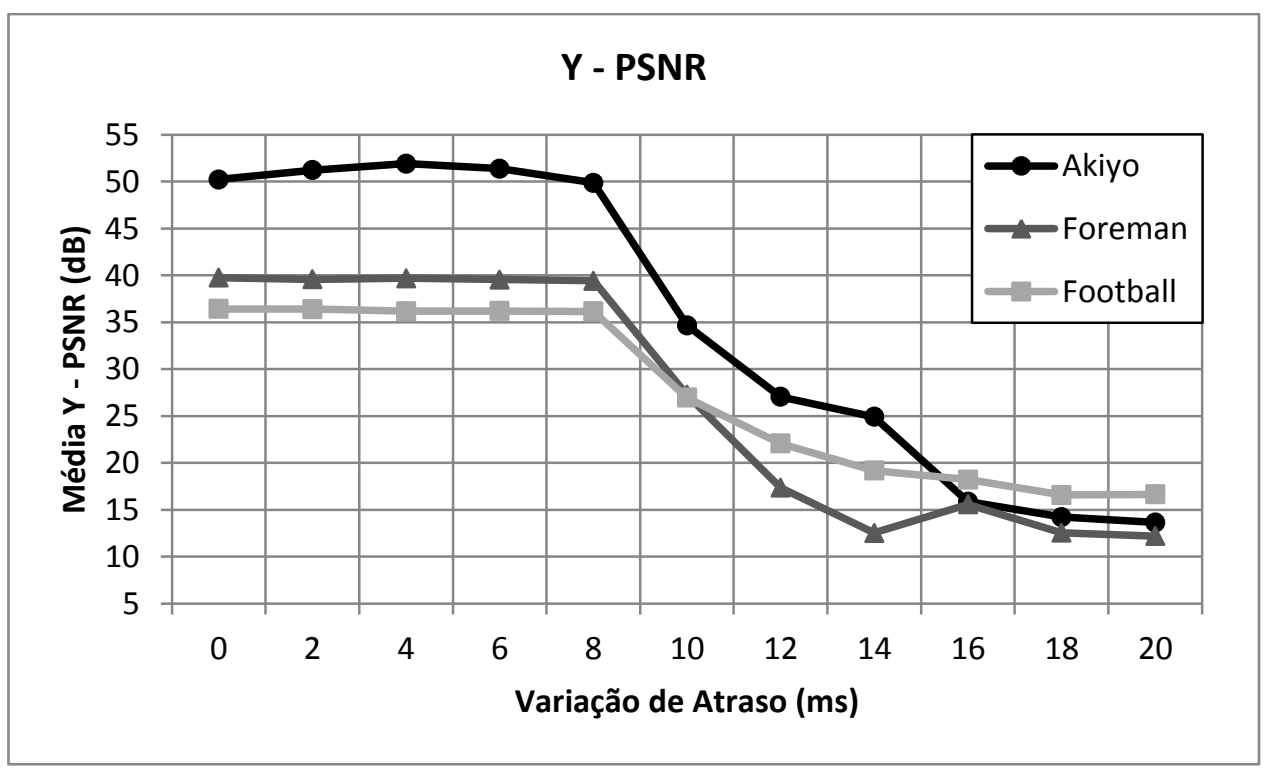

Figura 4.2: Gráfico da métrica PSNR (dB) por variação de atraso (ms) com valores entre 0 e 20 ms e atraso médio de 100 ms para os vídeos Akiyo, Foreman e Football. 


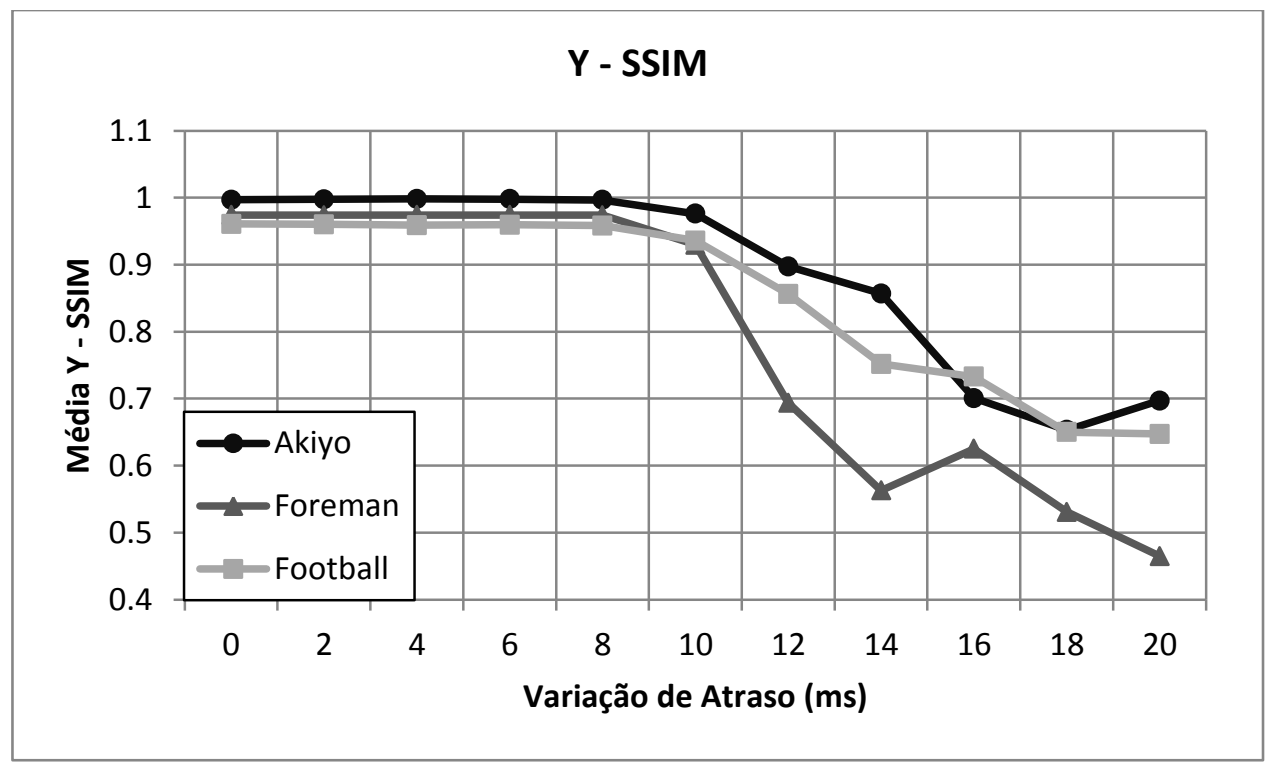

Figura 4.3: Gráfico da métrica SSIM por variação de atraso (ms) com valores entre 0 e 20 ms e atraso médio de 100 ms para os vídeos Akiyo, Foreman e Football.

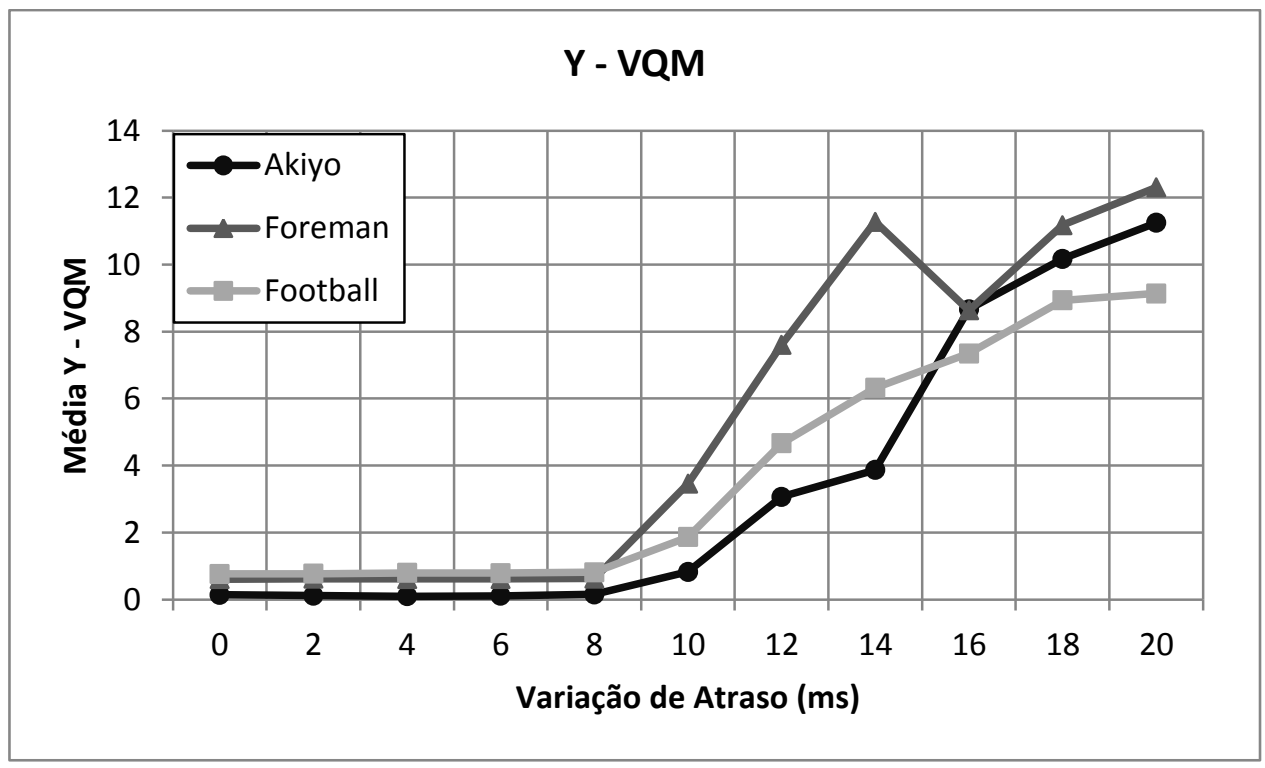

Figura 4.4: Gráfico da métrica VQM por variação de atraso (ms) com valores entre 0 e 20 ms e atraso médio de 100 ms para os vídeos Akiyo, Foreman e Football.

No caso da métrica PSNR e conforme a escala MOS (Mean Opinion Score), para um vídeo degradado ser considerado bom deve apresentar uma média acima de $30 \mathrm{~dB}$. Na Tabela 4.1 se observa a comparação entre os valores das médias PSNR e os correspondentes valores na escala MOS [KLAUE, RATHKE, \& WOLISZ, 2003]. 
Tabela 4.1: Conversão de PSNR para MOS.

\begin{tabular}{c|l}
\hline PSNR (dB) & \multicolumn{1}{c}{ MOS } \\
\hline$>37$ & 5 (Excelente) \\
$31-37$ & 4 (Bom) \\
$25-31$ & 3 (Razoável) \\
$20-25$ & 2 (Ruim) \\
$<20$ & 1 (Péssimo) \\
\hline
\end{tabular}

Segundo a Tabela 4.1, é possível observar na Figura 4.2 que os vídeos degradados entre 0 e $8 \mathrm{~ms}$ de variação de atraso são avaliados como excelente para Akiyo e Foreman, e bom para Football. Pode-se ressaltar que a partir de $8 \mathrm{~ms}$ de variação de atraso a qualidade dos vídeos começa a diminuir. Na faixa de 8 a $12 \mathrm{~ms}$ os vídeos degradados são avaliados como razoáveis, para finalmente serem avaliados como ruim ou péssimo a partir de $12 \mathrm{~ms}$. É importante ressaltar que os vídeos Akiyo, Foreman e Football apresentam diferentes conteúdos [ITU-T Rec. P.910, 2008], sendo a característica movimento importante na avaliação. Na maioria dos casos, quanto maior o movimento maior é o efeito da variação de atraso, ou seja, a qualidade de vídeo diminui mais rápidamente.

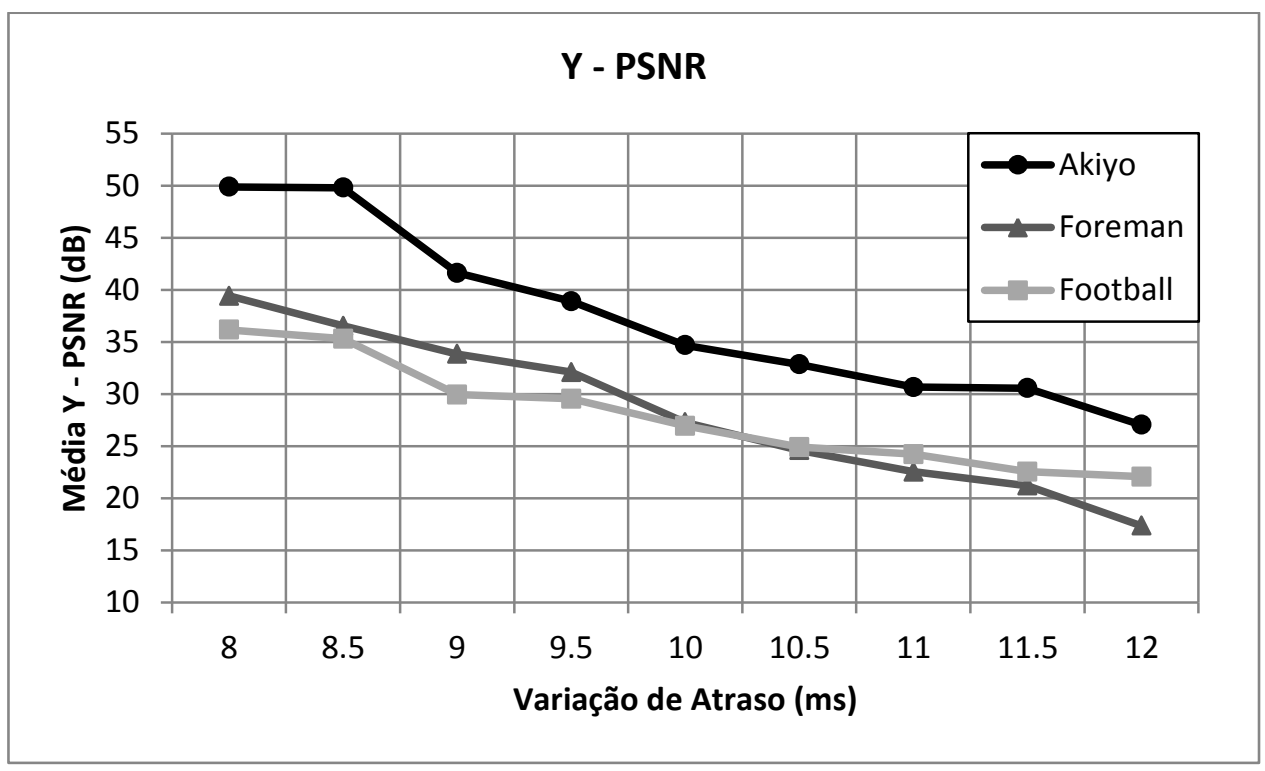

Figura 4.5: Gráfico da métrica PSNR (dB) por variação de atraso (ms) com valores entre 8 e 12 ms e atraso médio de 100 ms para os vídeos Akiyo, Foreman e Football. 


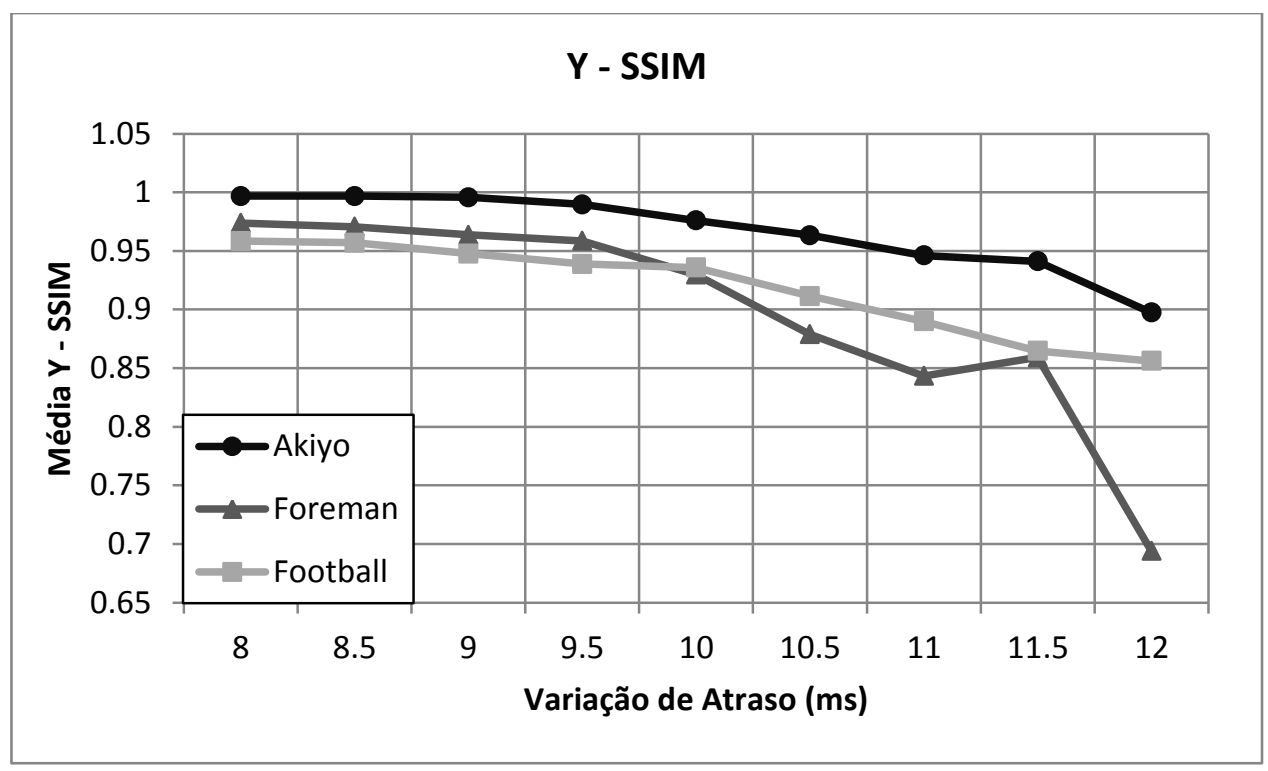

Figura 4.6: Gráfico da métrica SSIM por variação de atraso (ms) com valores entre 8 e 12 ms e atraso médio de 100 ms para os vídeos Akiyo, Foreman e Football.

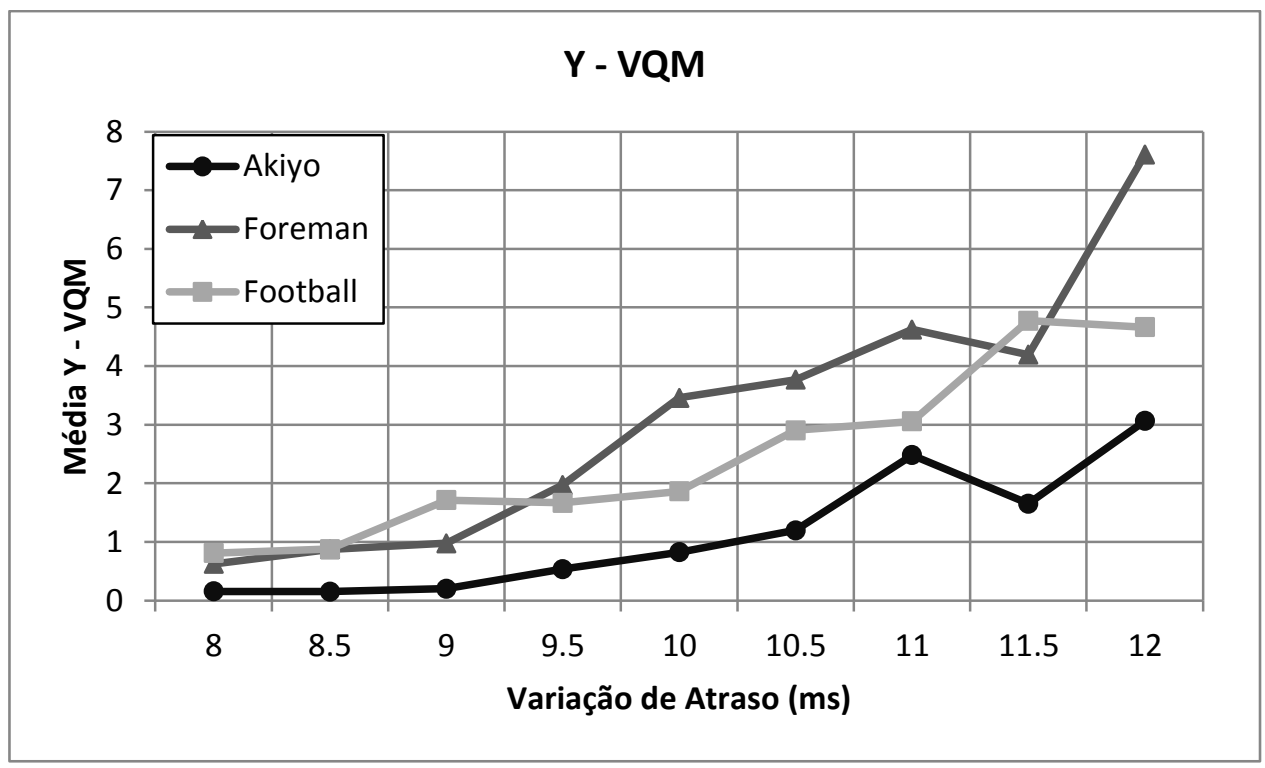

Figura 4.7: Gráfico da métrica VQM por variação de atraso (ms) com valores entre 8 e 12 ms e atraso médio de 100 ms para os vídeos Akiyo, Foreman e Football.

Na Figura 4.5 pode-se observar que os vídeos degradados entre 8 e 9.5 ms de variação de atraso são avaliados como excelente para Akiyo, bom para Foreman, e entre bom e razoável para Football. Ressalta-se que a qualidade dos vídeos diminui progressivamente à medida que a variação de atraso aumenta. Na faixa de 10 a $12 \mathrm{~ms}$ os vídeos degradados são avaliados como bom para Akiyo, e ruim para Foreman e Football. 


\subsection{Resultados da Avaliação Subjetiva}

Nesta seção, para efeitos de avaliação dos sinais de vídeo degradado em relação ao de referência, consideram-se as métricas subjetivas ACR e DCR [ITU-T Rec. P.910, 2008]. Foram consideradas 25 pessoas, sendo 15 homens e 10 mulheres na faixa de idade de 23 a 48 anos, não especialistas em processamento e avaliação de qualidade de vídeo. Adicionalmete, foi verificada a inexistência de problemas visuais que pudessem prejudicar a correta avaliação dos vídeos apresentados. Antes do início de cada teste foi realizada uma descrição acerca da avaliação e da escala de classificação para as métricas ACR e DCR, conforme a Tabela 4.2 e a Tabela 4.3 respectivamente. Realizou-se uma sessão de treino com os vídeos Crew e News [XPIH, 2010], com a finalidade de providenciar aos observadores a percepção de qualidade mínima e máxima dos vídeos com variação de atraso. Em seguida, foi realizada a sessão de teste com os vídeos Akiyo, Football e Foreman com um tempo de duração de 28 minutos para evitar fadiga e aborrecimento dos participantes.

Tabela 4.2: Escala de Classificação para a métrica ACR.

\begin{tabular}{c|l}
\hline Valor & Qualidade \\
\hline 5 & Excelente \\
4 & Bom \\
3 & Razoável \\
2 & Ruim \\
1 & Péssimo \\
\hline
\end{tabular}

Tabela 4.3: Escala de Classificação para a métrica DCR.

\begin{tabular}{c|l}
\hline Valor & \multicolumn{1}{|c}{ Degradação } \\
\hline 5 & Imperceptível \\
4 & Perceptível, mas não incômodo \\
3 & Ligeramente Incômodo \\
2 & Incômodo \\
1 & Muito Incômodo \\
\hline
\end{tabular}


$\mathrm{Na}$ sessão de teste, a degradação dos vídeos foi aleatoriamente apresentada aos participantes tanto para a métrica $\mathrm{ACR}$ quanto para a $\mathrm{DCR}$, com a finalidade de não polarizar a avaliação.

Com a finalidade de validar a avaliação dos observadores, foi realizada a fiabilidade dos participantes, através do método de seleção da recomendação ITU-R BT. 500-13 [ITU-R Rec. BT.500-13, 2012]. A fiabilidade dos participantes é avaliada qualitativamente pelo comportamento dos mesmos quando são reproduzidos os pares de vídeos referência/referência, as avaliações dos 25 observadores foram muito próximas de 5 . A seleção dos observadores se baseou num método de rejeição de pontuações incoerentes, para o qual se usa o coeficiente de curtose com a finalidade de saber se a distribuição para cada apresentação é uma distribuição normal ou não. Caso a distribuição seja normal, o valor do coeficiente de curtose se encontra entre 2 e 4, caso contrário a distribuição não é considerada normal. A partir do tipo de distribuição são estabelecidas duas condições que devem ser cumpridas para rejeitar o observador. A primeira condição está relacionada ao número de vezes em que o observador atribui avaliações que caem fora do intervalo de confiança. Se tal taxa for superior a 5\% das vezes, o observador pode ser rejeitado. Já na segunda condição, se os resultados estiverem dispersos em ambas as extremidades da distribuição dos resultados de qualidade, o observador pode ser rejeitado caso a taxa seja menor do que $30 \%$. Se ambas as condições apontarem a possibilidade de rejeição o observador é rejeitado. O detalhamento desse método encontra-se descrito na recomendação ITU-R BT. 500-13 [ITU-T Rec. BT.500-13, 2012]. Os resultados obtidos do método de seleção de observadores para a primeira e segunda condições foram de 0,0303 e 1,0000 respectivamente. Após a aplicação do referido método, verificou-se que nenhum dos observadores foi rejeitado.

Nas Figuras 4.8, 4.9, 4.10, 4.11, 4.12 e 4.13 são apresentados os intervalos de confiança de 95\% [ITU-R Rec. BT.500-13, 2012] para as médias ACR e DCR. Pode-se ressaltar que para a métrica ACR o intervalo de confiança é menor a partir de $14 \mathrm{~ms}$, ou seja, os observadores tiveram uma maior precisão na avaliação de qualidade dos vídeos degradados conforme aumento da variação de atraso, na faixa entre 14 e $20 \mathrm{~ms}$. No caso específico de Akiyo e Foreman os 25 observadores fizeram a mesma avaliação para o vídeo degradado com variação de atraso de 18 e $20 \mathrm{~ms}$. Para a métrica DCR o intervalo de confiança é maior para variação de atraso entre 10 e 16 ms para Akiyo e Foreman, e entre 10 e 20 ms para Football. Ou seja, os observadores foram mais precisos na avaliação da degradação dos 
vídeos com menor e maior degradação para Akiyo e Foreman, e com menor degradação para Football.

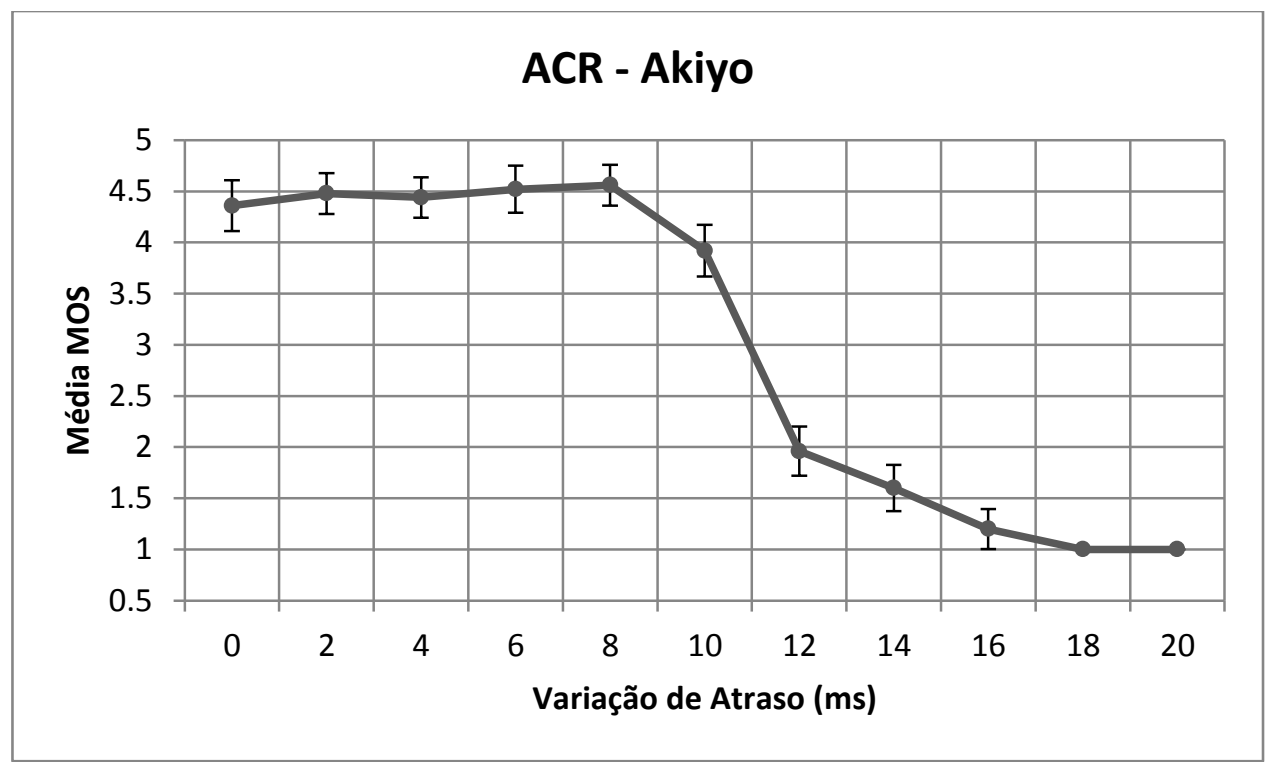

Figura 4.8: Gráfico da métrica ACR com intervalos de confiança de 95\% para variações de atraso com valores entre 0 e $20 \mathrm{~ms}$ e atraso médio de 100 ms para o vídeo Akiyo.

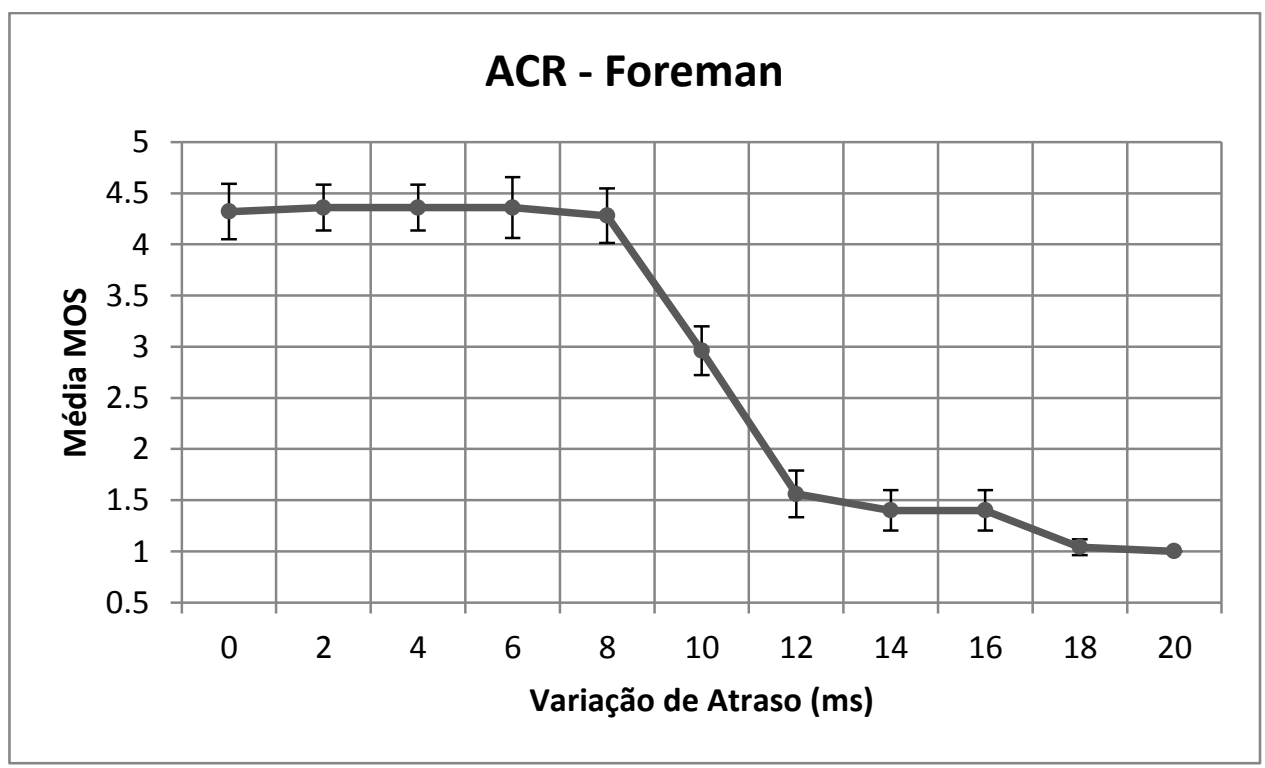

Figura 4.9: Gráfico da métrica ACR com intervalos de confiança de 95\% para variações de atraso com valores entre 0 e $20 \mathrm{~ms}$ e atraso médio de 100 ms para o vídeo Foreman. 


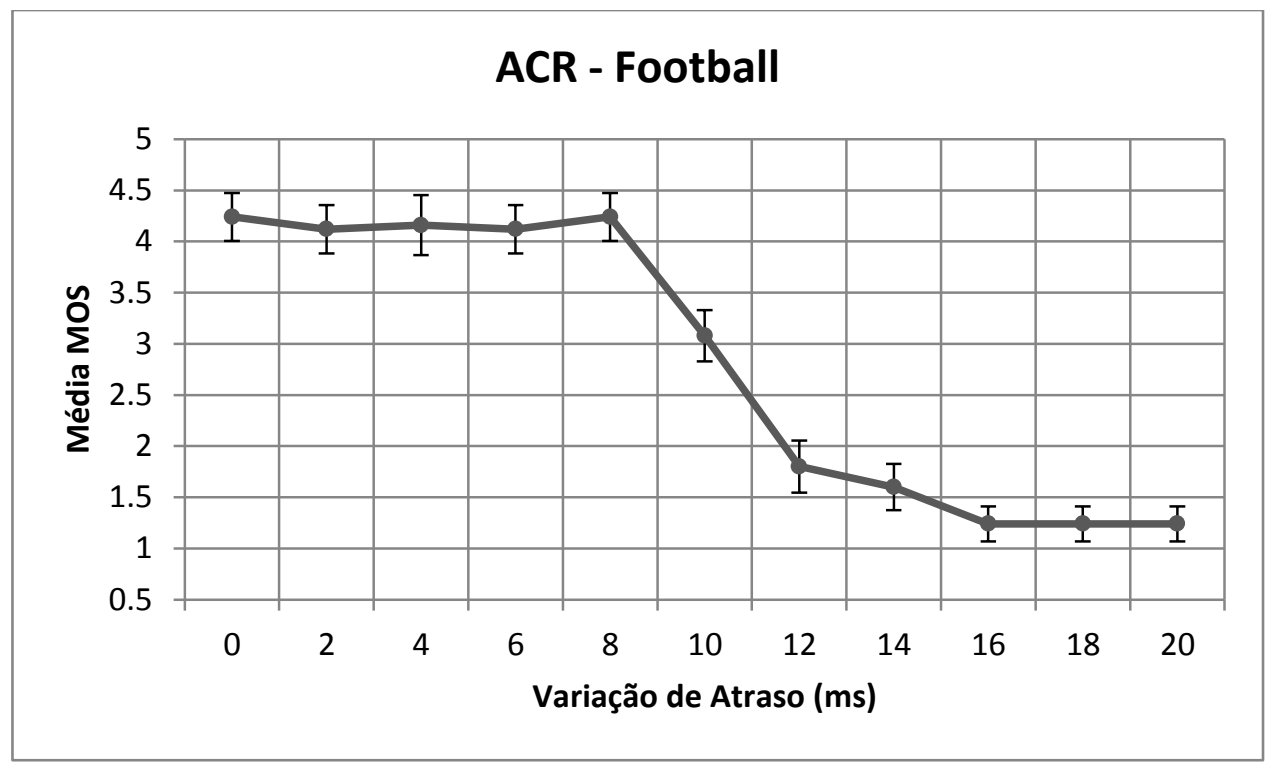

Figura 4.10: Gráfico da métrica ACR com intervalos de confiança de 95\% para variações de atraso com valores entre 0 e 20 ms e atraso médio de 100 ms para o vídeo Football.

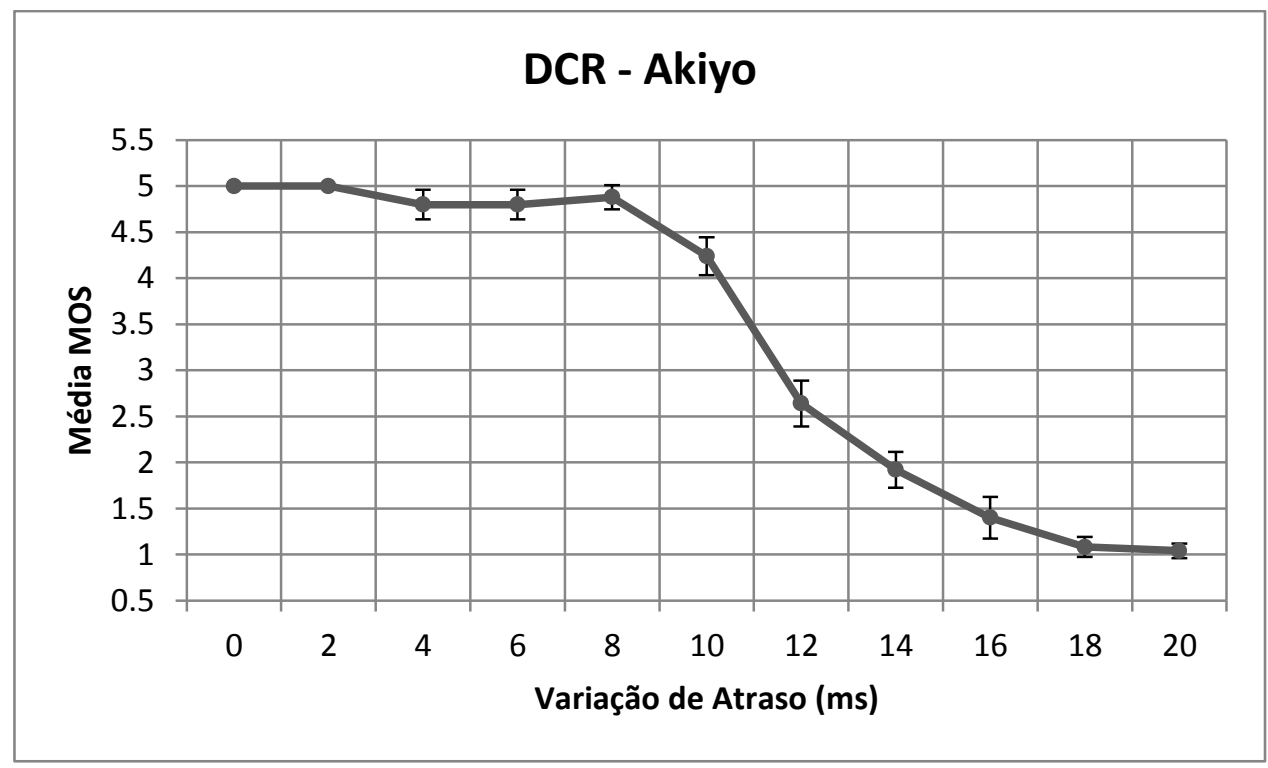

Figura 4.11: Gráfico da métrica DCR com intervalos de confiança de $95 \%$ para variações de atraso com valores entre 0 e 20 ms e atraso médio de 100 ms para o vídeo Akiyo.

Os intervalos de confiança para a métrica ACR são maiores do que os intervalos de confiança para a métrica DCR. Isso pode ser explicado pelo fato de que na métrica ACR não se usa o vídeo de referência. No caso da métrica DCR, antes da reprodução de cada vídeo degradado é reproduzido o vídeo de referência o que facilita a avaliação dos observadores. 
Como desvantagem, o tempo de duração do teste para a métrica DCR é maior do que o teste para a ACR.

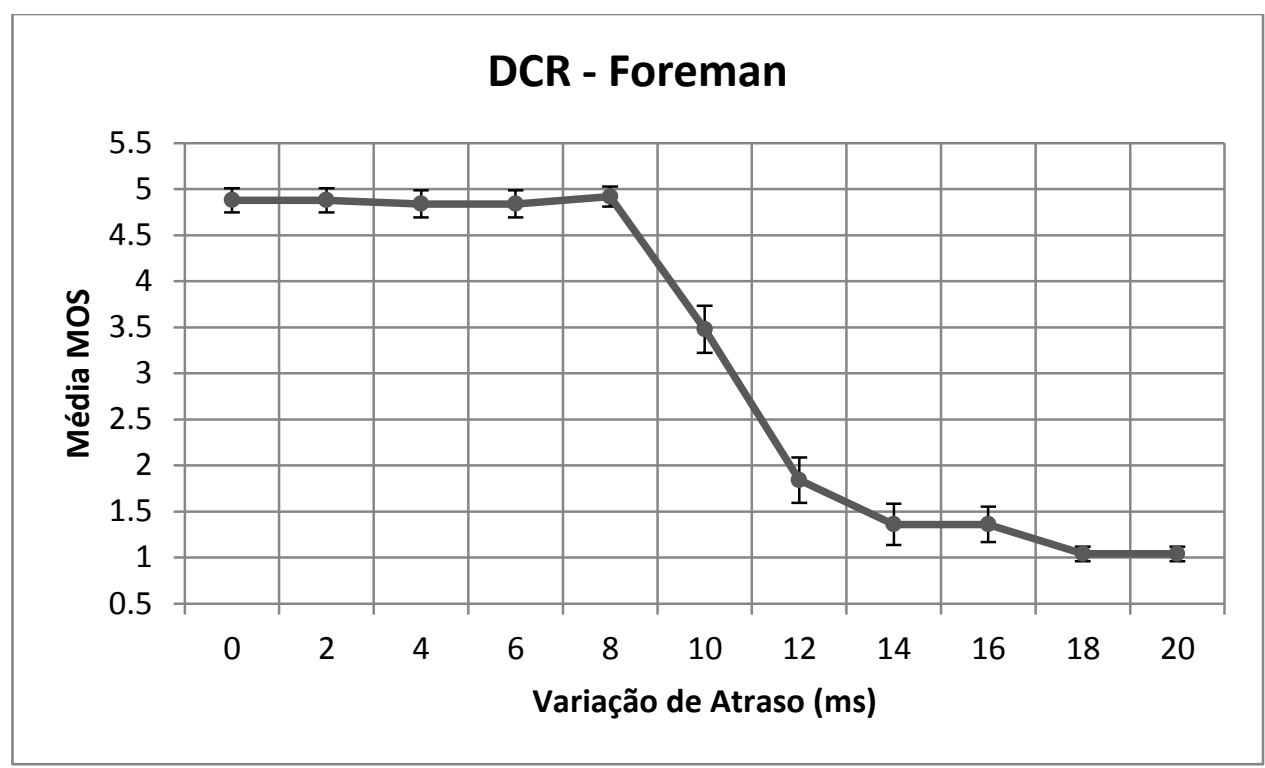

Figura 4.12: Gráfico da métrica DCR com intervalos de confiança de 95\% para variações de atraso com valores entre 0 e $20 \mathrm{~ms}$ e atraso médio de 100 ms para o vídeo Foreman.

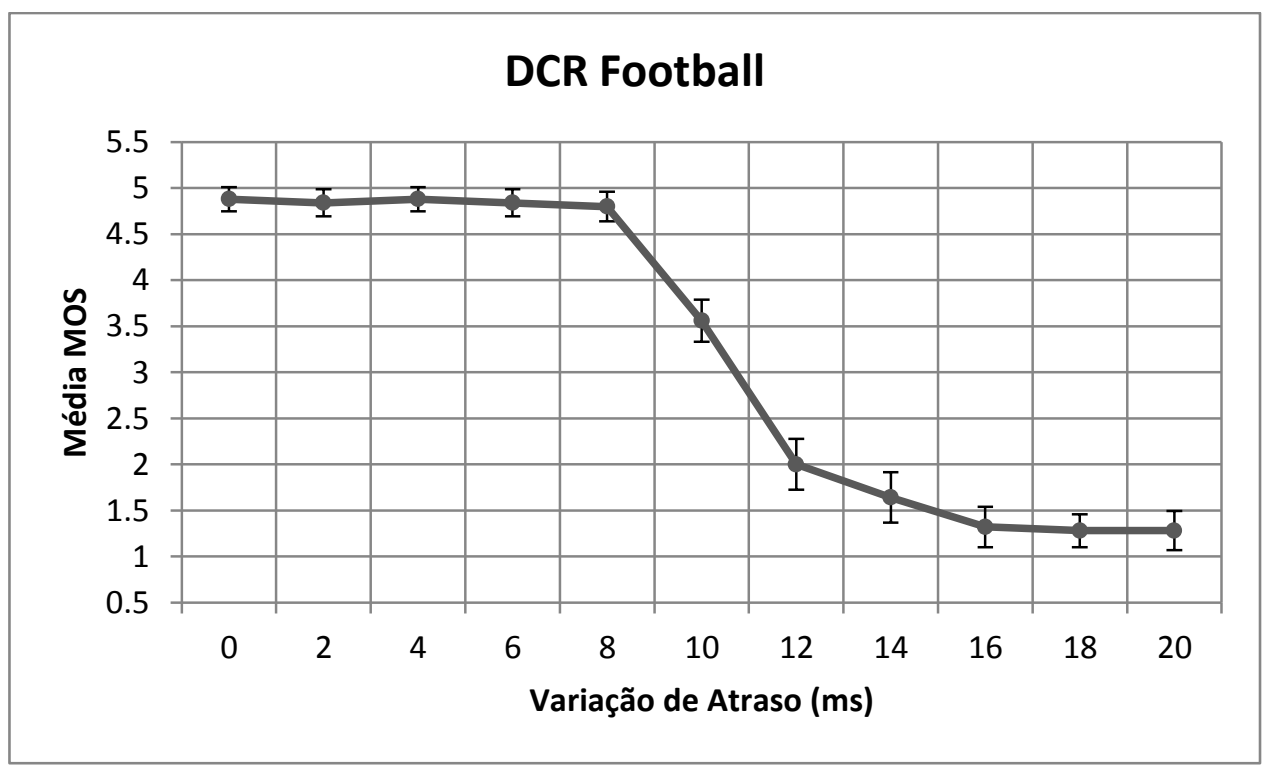

Figura 4.13: Gráfico da métrica DCR com intervalos de confiança de 95\% para variações de atraso com valores entre 0 e $20 \mathrm{~ms}$ e atraso médio de $100 \mathrm{~ms}$ para o vídeo Football. 
Nas métricas subjetivas ACR e DCR os observadores avaliaram a qualidade e a degradação dos vídeos respectivamente. A qualidade de vídeo é menor em função do aumento da variação de atraso, conforme os resultados mostrados na Figura 4.14 usando a métrica ACR, para os vídeos Akiyo, Foreman e Football com variação de atraso entre 0 e 20 ms e com atraso médio de 100 ms. Na Figura 4.15 são apresentados os resultados usando a métrica DCR para os mesmos vídeos e com a mesma faixa de valores de variação de atraso e atraso médio. Neste caso, quanto maior é a variação de atraso, menor é o valor obtido na escala MOS e mais intensa se torna a degradação do vídeo avaliado pelos observadores.

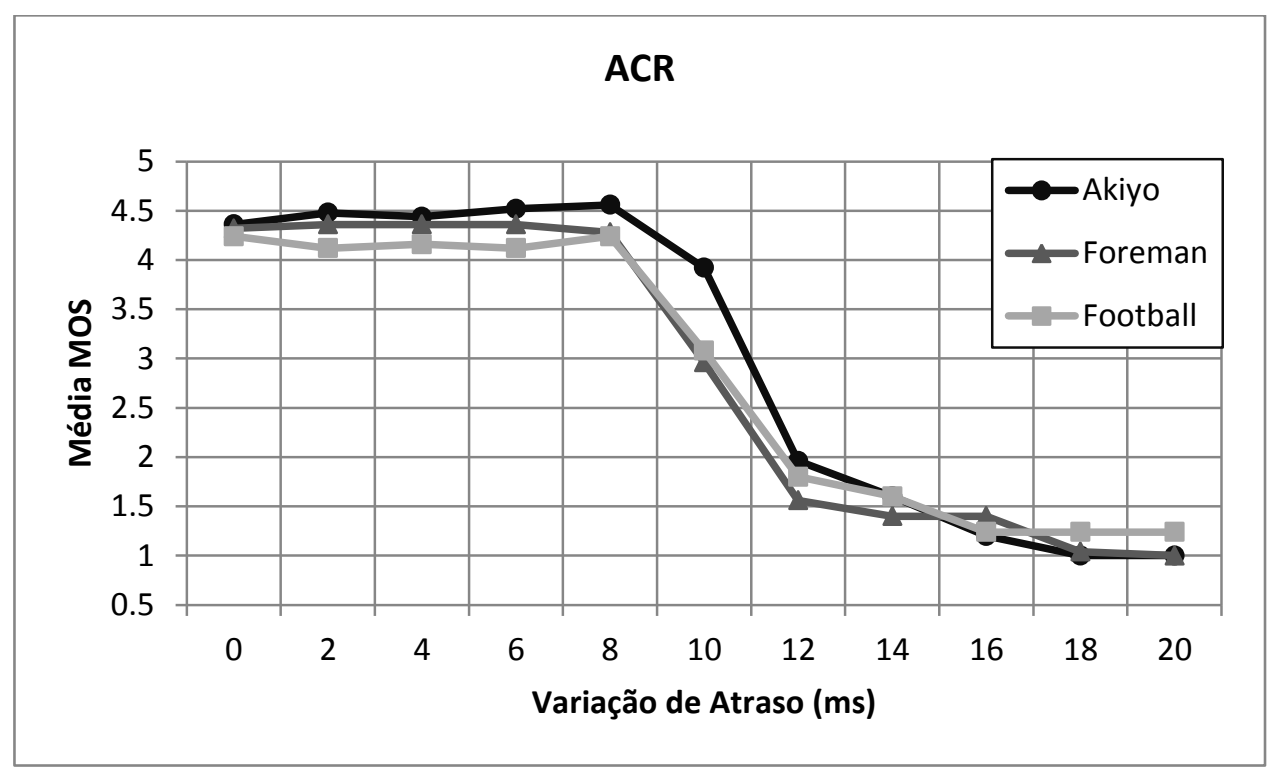

Figura 4.14: Gráfico da métrica ACR por variação de atraso (ms) com valores entre 0 e 20 ms e atraso médio de 100 ms para os vídeos Akiyo, Foreman e Football.

Segundo a Tabela 4.2, é possível observar na Figura 4.14 que os vídeos degradados entre 0 e $8 \mathrm{~ms}$ de variação de atraso foram avaliados pelos observadores como excelente e bom para os vídeos Akiyo, Foreman e Football, pode-se ressaltar que a partir de $8 \mathrm{~ms}$ de variação de atraso a qualidade dos vídeos começa a diminuir. Na faixa de 8 a $12 \mathrm{~ms}$ os vídeos degradados são avaliados como bom e razoável, para finalmente serem avaliados como ruim ou péssimo a partir de $12 \mathrm{~ms}$. É importante ressaltar que os vídeos Akiyo, Foreman e Football são de categoria A, B e E respectivamente [ITU-T Rec. P.910, 2008], em que a categoria A, corresponde a uma pessoa com movimentos limitados, a categoria B coincide com uma pessoa, gráficos e maiores detalhes e, finalmente, a categoria E corresponde a muito movimento. 


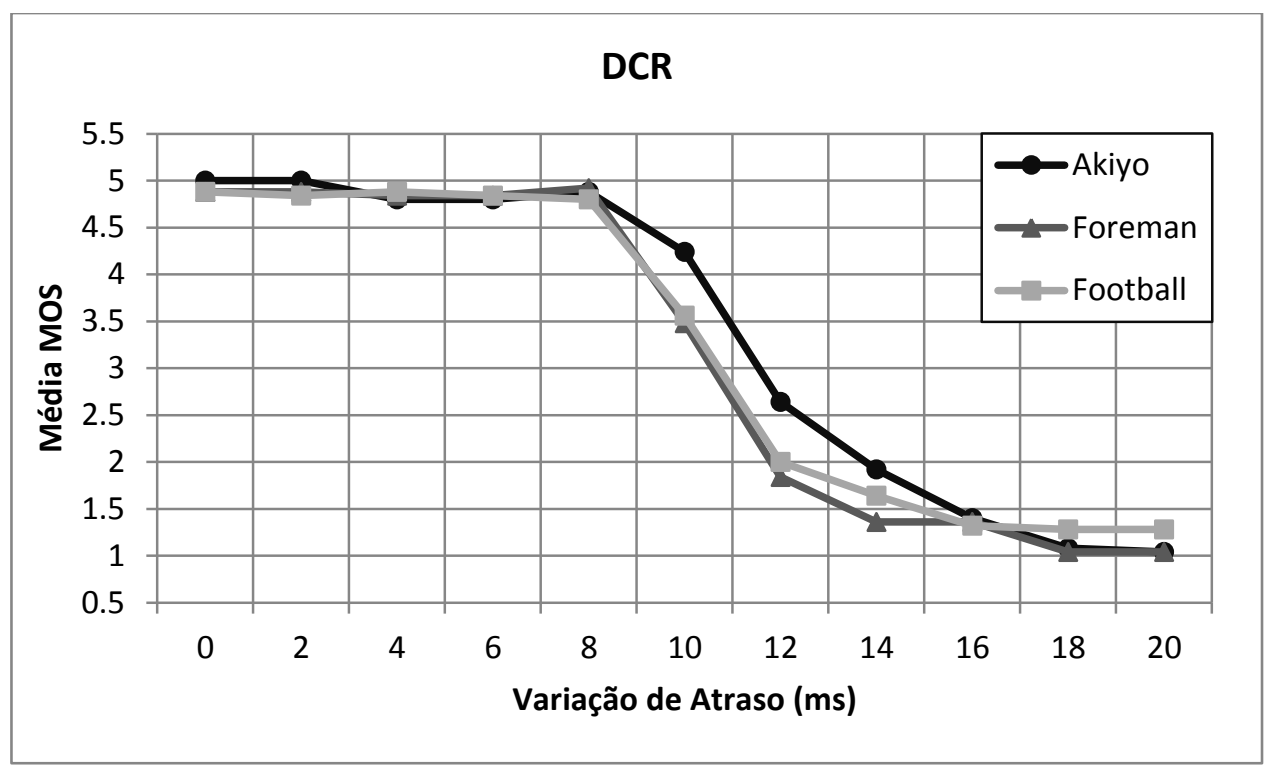

Figura 4.15: Gráfico da métrica DCR por variação de atraso (ms) com valores entre 0 e 20 ms e atraso médio de 100 ms para os vídeos Akiyo, Foreman e Football.

Na Figura 4.15 pode-se observar que os vídeos degradados entre 0 e 8 ms de variação de atraso foram avaliados com degradação Imperceptível Akiyo, Foreman e Football. Nota-se que a partir de $8 \mathrm{~ms}$ de variação de atraso a média MOS começa a diminuir, progresivamente indicando que a degradação percebida pelos observadores aumenta. Os participantes avaliaram os vídeos degradados na faixa de 8 a $12 \mathrm{~ms}$ como perceptível e ligeramente incômodo para Akiyo, ligeramente incômodo e incômodo para Foreman, e perceptível e incômodo para Football. Finalmente os vídeos degradados foram avaliados pelos observadores como incômodo e muito incômodo a partir de $12 \mathrm{~ms}$.

\subsection{Comparação entre métricas}

Esta seção foi dividida em duas partes para efeito de comparação das métricas. Na primeira, considerou-se o coeficiente de correlação para a comparação das métricas objetivas com as métricas subjetivas. Para poder comparar essas métricas, foi necessária normalização dos resultados obtidos a partir de cada uma. Para a normalização entre métricas objetivas e subjetivas foi usado o método de projeção linear. Na segunda parte, foi utilizada a análise de variância (ANOVA - analysis of variance) para a comparação entre métricas subjetivas.

Na Tabela 4.4 e Tabela 4.5 são apresentados os resultados da comparação das métricas objetivas PSNR, SSIM e VQM com as métricas subjetivas ACR e DCR respectivamente. 
Tem-se como finalidade principal mostrar o desempenho das métricas objetivas como projeção linear em relação às subjetivas, usadas no presente trabalho, através dos coeficientes de correlação de Pearson e Spearman, e também através dos erros de predição e quadrático médio.

Tabela 4.4: Desempenho das métricas objetivas em relação à métrica subjetiva ACR.

\begin{tabular}{l|c|c|c|c}
\hline Métrica/Vídeo & $\begin{array}{c}\text { C.C. de } \\
\text { Pearson }\end{array}$ & $\begin{array}{c}\text { C.C. de } \\
\text { Spearman }\end{array}$ & $\begin{array}{c}\text { Erro de } \\
\text { Predição } \\
\text { Linear }\end{array}$ & $\begin{array}{c}\text { Erro } \\
\text { RMS }\end{array}$ \\
\hline PSNR/Akiyo & 0.98 & 0.88 & 0.12 & 0.34 \\
SSIM/Akiyo & 0.94 & 0.87 & 0.32 & 0.54 \\
VQM/Akiyo & -0.92 & -0.88 & 0.41 & 0.61 \\
\hline PSNR/Foreman & 1.00 & 0.93 & 0.01 & 0.11 \\
SSIM/Foreman & 0.97 & 0.93 & 0.16 & 0.38 \\
VQM/Foreman & -0.98 & -0.88 & 0.09 & 0.29 \\
\hline PSNR/Football & 0.99 & 0.92 & 0.02 & 0.14 \\
SSIM/Football & 0.93 & 0.94 & 0.25 & 0.47 \\
VQM/Football & -0.96 & -0.92 & 0.14 & 0.36 \\
\hline
\end{tabular}

Tabela 4.5: Desempenho das métricas objetivas em relação à métrica subjetiva DCR.

\begin{tabular}{l|c|c|c|c}
\hline Métrica/Vídeo & $\begin{array}{c}\text { C.C. de } \\
\text { Pearson }\end{array}$ & $\begin{array}{c}\text { C.C. de } \\
\text { Spearman }\end{array}$ & $\begin{array}{c}\text { Erro de } \\
\text { Predição } \\
\text { Linear }\end{array}$ & $\begin{array}{c}\text { Erro } \\
\text { RMS }\end{array}$ \\
\hline PSNR/Akiyo & 0.98 & 0.92 & 0.12 & 0.33 \\
SSIM/Akiyo & 0.96 & 0.91 & 0.25 & 0.48 \\
VQM/Akiyo & -0.94 & -0.92 & 0.33 & 0.55 \\
\hline PSNR/Foreman & 1.00 & 0.89 & 0.02 & 0.14 \\
SSIM/Foreman & 0.97 & 0.85 & 0.17 & 0.39 \\
VQM/Foreman & -0.98 & -0.85 & 0.10 & 0.30 \\
\hline PSNR/Football & 0.99 & 0.97 & 0.03 & 0.16 \\
SSIM/Football & 0.94 & 0.99 & 0.35 & 0.56 \\
VQM/Football & -0.96 & -0.97 & 0.20 & 0.42 \\
\hline
\end{tabular}


Pode-se perceber segundo os resultados da Tabela 4.4 e Tabela 4.5, que nenhuma das métricas objetivas foi melhor que a PSNR pelo que apresenta melhor eficiência computacional entre as métricas objetivas aqui consideradas, como foi concluído pelo VQEG [VQEG, 2000] e em [FONSECA, 2008]. Os valores dos coeficientes de correlação para a métrica VQM são negativos. Isso ocorre já que a curva de avaliação de qualidade tende a apresentar valores crescentes à medida em que a variação de atraso aumenta, conforme mostra a Figura 4.4 e, ao contrário do que ocorre com as métricas subjetivas ACR e DCR. A qualidade de aproximação foi melhor para a métrica PSNR devido ao erro de predição, menor do que o das outras métricas objetivas. Também pode-se observar que os vídeos Akiyo e Football apresentam os maiores valores de erro quadrático médio, ressaltando que os erros apresentados nestes vídeos com movimento limitado e com muito movimento não são detectáveis pelo sistema visual humano como foram detetados com as métricas PSNR, SSIM e VQM. Ou seja, as métricas objetivas não levam em consideração todos os efeitos do sistema visual humano. A métrica VQM é a mais esperta em utilizar as características do sistema visual humano (veja Apêndice $\mathrm{A}$ ), em comparação com às outras duas métricas objetivas.

$\mathrm{Na}$ segunda parte foram comparadas as médias das métricas subjetivas para Akiyo, Foreman e Football e, também foram comparados os vídeos para as métricas subjetivas ACR e DCR. Finalmente foram comparados os resultados da variação de atraso para as métricas subjetivas. Na comparação foi utilizado o método ANOVA para avaliar a significância entre grupos, sendo obtidos o nível de significância ou valor $\mathrm{P}$ para o teste $\mathrm{F}$ e o valor de $\mathrm{F}$. $H_{0}$ representa a afirmação de que todas as médias são iguais. Finalmente, com os valores obtidos, é avaliada a hipótese nula $H_{0}$ se o valor $\mathrm{P}$ for menor do que o nível de significância $\alpha$. Caso contrário, se o valor de $\mathrm{F}$ obtido for maior do que o $\mathrm{F}$ crítico, então $H_{0}$ é rejeitada, ou seja, existe diferença significativa entre os grupos. F crítico é obtido através da tabela de valores críticos para a distribuição $\mathrm{F}$, considerando os graus de liberdade no numerador e denominador.

Na Tabela 4.6 são apresentados os resultados da comparação entre as médias ACR e DCR, na Tabela 4.7 os resultados da comparação dos vídeos por categorias e, finalmente, na Tabela 4.8 os resultados da comparação da variação de atraso. Todos os resultados foram avaliados com o nível de significância $\alpha=0,05$. 
Tabela 4.6: Comparação entre as médias das métricas ACR e DCR com $\alpha=0,05$.

\begin{tabular}{l|c|c|c}
\hline \multicolumn{1}{c|}{ Vídeos } & Valor P & F & F crítico \\
\hline Akiyo & 0.64 & 0.23 & 4.35 \\
Foreman & 0.66 & 0.19 & 4.35 \\
Football & 0.57 & 0.34 & 4.35 \\
\hline
\end{tabular}

Tabela 4.7: Comparação entre as médias das cenas reproduzidas com $\alpha=0,05$.

\begin{tabular}{c|c|c|c}
\hline Métrica & Valor P & F & F crítico \\
\hline ACR & 0.95 & 0.05 & 3.32 \\
DCR & 0.96 & 0.04 & 3.32 \\
\hline
\end{tabular}

Tabela 4.8: Comparação entre as médias de variação de atraso com $\alpha=0,05$.

\begin{tabular}{c|c|c|c}
\hline $\begin{array}{c}\text { Variação de } \\
\text { Atraso (ms) }\end{array}$ & Valor P & F & F crítico \\
\hline $0-8$ & 1.00 & 0.02 & 2.76 \\
$8-12$ & 0.00 & 69.24 & 3.68 \\
$12-16$ & 0.00 & 10.57 & 3.68 \\
$18-20$ & 0.85 & 0.04 & 4.96 \\
\hline
\end{tabular}

Como se pode observar, na Tabela 4.6 a hipótese nula $H_{0}$ não foi rejeitada, ou seja, não existe diferença significativa entre as médias das métricas ACR e DCR para os vídeos Akiyo, Foreman e Football. Mesmo que as métricas subjetivas usadas sejam métodos que avaliam a qualidade e a degradação dos vídeos, pode-se dizer que existe alguma relação entre a avaliação de qualidade e a avaliação de degradação dos vídeos feita pelos observadores. $\mathrm{Na}$ Tabela 4.7 não existe diferença significativa entre as médias das cenas reproduzidas que apresentam conteúdos diferentes, sendo possível dizer que a variação de atraso é percebida pelos observadores sem fazer diferença do conteúdo dos vídeos.

Finalmente, na Tabela 4.8 a hipótese nula $H_{0}$ é rejeitada para os intervalos de 8 a $12 \mathrm{ms,}$ e 12 a 16 ms, em que existe diferença significativa entre as médias da variação de atraso. Portanto, pode-se dizer que existe uma maior diferença no intervalo de 8 a $12 \mathrm{~ms}$, em que foram comparadas as médias da variação de atraso de 8, 10 e 12 ms. É possível perceber que 
a qualidade dos vídeos diminui rapidamente. No entanto, para os intervalos de 0 a $8 \mathrm{~ms}$, e 18 a 20 ms não existe diferença significativa, devido à avaliação dos observadores ter sido excelente ou péssimo respectivamente nesses intervalos de variação de atraso. 


\section{Capítulo 5}

\section{Conclusões e trabalhos futuros}

Este trabalho foi focado na realização de avaliações objetiva e subjetiva de sequências de vídeo para diferentes valores de variação de atraso, e na comparação das respectivas médias obtidas. Foram consideradas as métricas objetivas PSNR, SSIM e VQM que contemplam apenas a componente de luminância (Y) e as métricas subjetivas ACR e DCR. Uma base de dados de vídeos degradados com diferentes valores de variação de atraso foi gerada a partir da transmissão de vídeos de referência em um cenário de rede totalmente isolada.

\subsection{Conclusões}

A avaliação de qualidade de vídeo é afetada diretamente pelos fatores de degradação de rede como a variação de atraso, conforme os resultados obtidos a partir das métricas objetivas PSNR, SSIM e VQM e das métricas subjetivas ACR e DCR.

Analisou-se o efeito da varias distribuições uniformes com atraso médio de $100 \mathrm{~ms}$ e desvios máximos diferentes. Para o intervalo de desvios máximos entre 8 e $12 \mathrm{~ms}$ a queda de qualidade é mais acentuada. Na rede de pacotes, a variação do atraso é um parâmetro mais importante do que o valor específico do atraso. De acordo com os testes, o limiar de atraso perceptível é de $8 \mathrm{~ms}$, no intervalo de atraso [92 ms, $108 \mathrm{~ms}$ ], e com $12 \mathrm{~ms}$ de desvio máximo unilateral a qualidade cai a um nível inaceitável no intervalo de atraso [88ms, $112 \mathrm{~ms}$ ]. O desvío máximo de $12 \mathrm{~ms}$ é um limiar de qualidade péssima.

$\mathrm{Na}$ avaliação subjetiva, através dos intervalos de confiança, verificou-se que os observadores tiveram uma maior precisão na avaliação na medida em que a variação de atraso aumenta, especificamente a partir de $14 \mathrm{~ms}$ de variação de atraso, quando usada a 
métrica ACR que avalia a qualidade de vídeo. Para a métrica DCR, que avalia a degradação do vídeo, os observadores foram menos precisos na avaliação para a variação de atraso compreendida no intervalo entre 10 e $16 \mathrm{~ms}$. Também verificou-se que nas cenas com muito movimento foram menos precisos do que nas cenas com movimento limitado a partir de 16 ms. Da mesma forma, os valores de intervalos de confiança para a métrica ACR são maiores do que os valores para a métrica DCR, devido ao fato da métrica ACR não usar o vídeo de referência, como ocorre na métrica DCR, facilitando a avaliação dos observadores.

$\mathrm{Na}$ comparação entre métricas objetivas e subjetivas nota-se que a qualidade de aproximação é melhor para a métrica PSNR, que apresenta melhor eficiência computacional do que as outras métricas objetivas. As métricas objetivas não são capazes de substituir às métricas subjetivas e sim complementar a avaliação subjetiva da qualidade do vídeo. Além disso, o resultado da comparação entre métricas subjetivas não apresentou diferenças significativas, indicando que existe alguma relação entre a avaliação de qualidade e a avaliação de degradação.

\subsection{Trabalhos Futuros}

No intuito de dar continuidade a este trabalho, são propostos alguns temas relacionados que podem ser desenvolvidos empregando o mesmo cenário de testes.

- Realizar as avaliações objetiva e subjetiva das sequências de vídeo codificadas para diferentes valores de taxa de codificação no padrão H.264.

- Avaliar a qualidade de vídeos com maior resolução como definição padrão ou alta definição, e com diferentes conteúdos.

- Estabelecer o fator de degradação de variação de atraso com diferentes tipos de distribuições.

- Propor uma nova métrica de avaliação subjetiva, com as sugestões coletadas pelos observadores.

- Implementar um algoritmo de controle baseado na qualidade de vídeo para otimizar os recursos de uma rede IP.

- Realizar a avaliação de qualidade de vídeo em um cenário sem fio com diferentes valores de variação de atraso. 


\section{Apêndice A}

\section{Sistema Visual Humano}

Nesta seção serão apresentados aspectos relevantes do sistema visual humano, componentes que são bem compreendidos e aceitos pela comunidade da ciência da visão [WINKLER, 2005]. Estes componentes são importantes na parametrização dos algoritmos de processamento de vídeo e, mais especificamente, ao projeto de métricas objetivas de qualidade de vídeo.

A luz visível possibilita o sentido da visão, faz parte do espectro de radiação electromagnética e se encontra entre os 380 e 760nm de comprimento. O olho humano apresenta a característica da sensibilidade à luz e adicionalmente um sistema de lentes, fazendo possível a formação de imagens neste orgão óptico. A Figura A.1 ilustra a secção transversal de um olho.

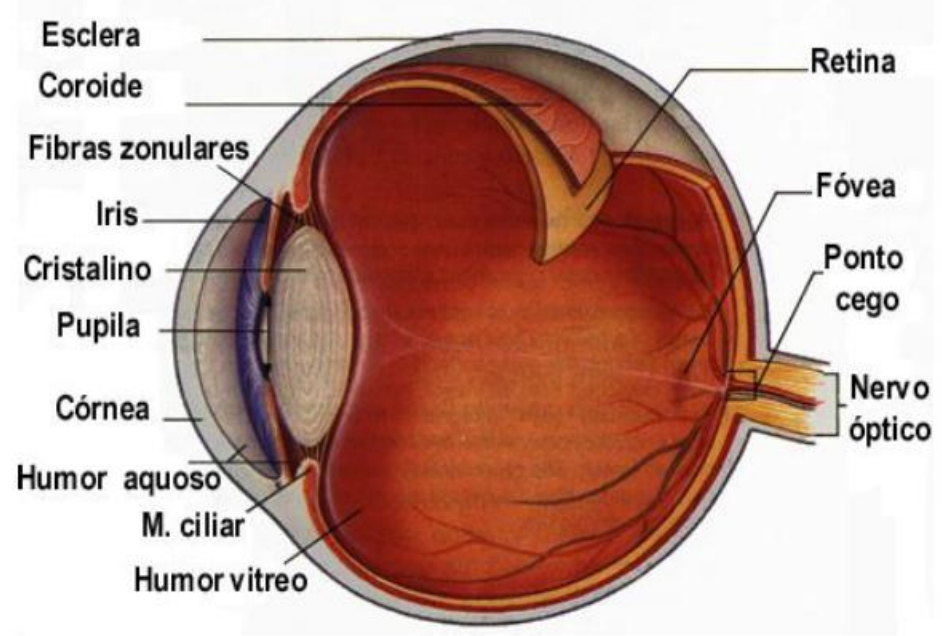

Figura A.1: Secção transversal do olho. 
O olho possui um mecanismo de focalização do objeto de interesse realizado pelo cristalino que é uma lente biconvexa e pela córnea, um sistema de lentes que refratam a luz realizado pela córnea e o cristalino, pupila de diâmetro regulável para controlar a quantidade de luz que passa para a retina, filme de revelação rápida das imagens e um sistema de proteção e de manuntenção de transparencia do aparelho ocular. As células sensíveis à luz encontram-se na retina e através de um processo fotoquímico, mais de cem milhões de células fotorreceptoras transformam ou transduzem as ondas luminosas em impulsos eletroquímicos, os quais são levados através do nervo ótico para serem decodificados pelo cérebro. [NISHIDA, 2007].

Existem dois tipos de células fotorreceptoras na retina: os cones e bastonetes. A retina humana possui aproximadamente 120 milhões de bastonetes e 6 milhões de cones. Os cones são responsáveis pela visão em condições normais de luz, enquanto os bastonetes são responsáveis pela visão em condições de baixa luminosidade e pela detecção de movimentos súbitos e formas sem cor, chamada de visão periférica. Existem três tipos de células cones, denominados de curto $(\mathrm{S})$, médio $(\mathrm{M})$ e longo $(\mathrm{L})$, dividem a imagem projetada sobre a retina em três fluxos visuais entendidos como as componentes de cor vermelha, verde e azul [CORNSWEET, 1970].

Os principais componentes do sistema visual humano para a parametrização dos algoritmos de processamento de vídeo são enumerados a seguir.

\section{- Percepção do Contraste}

A sensibilidade do sistema visual humano é de fundamental importância para a avaliação subjetiva de qualidade de vídeo. A capacidade de distinguir contraste entre áreas adjacentes determina o grau de percepção dos detalhes de uma cena [CAMPBELL \& MAFFEI, 1974]. A função de sensibilidade ao contraste (CSF Contrast Sensitivity Function) modela a variação na sensibilidade do sistema visual humano em função das frequências espaciais e temporais que estão presentes no estímulo visual [SANTOS \& SIMAS, 2001]. A CSF é modelada como uma função passa-banda invariante ao deslocamento mostrada na Figura A.2. A maioria dos algoritmos de avaliação de qualidade implementam uma versão passa-baixa de CSF. O contraste é definido conforme a equação: 


$$
C=\frac{L_{\max }-L_{\min }}{L_{\max }+L_{\min }}
$$

em que $L_{\max }$ é a função de máxima luminosidade e $L_{\min }$ é a função de mínima luminosidade.

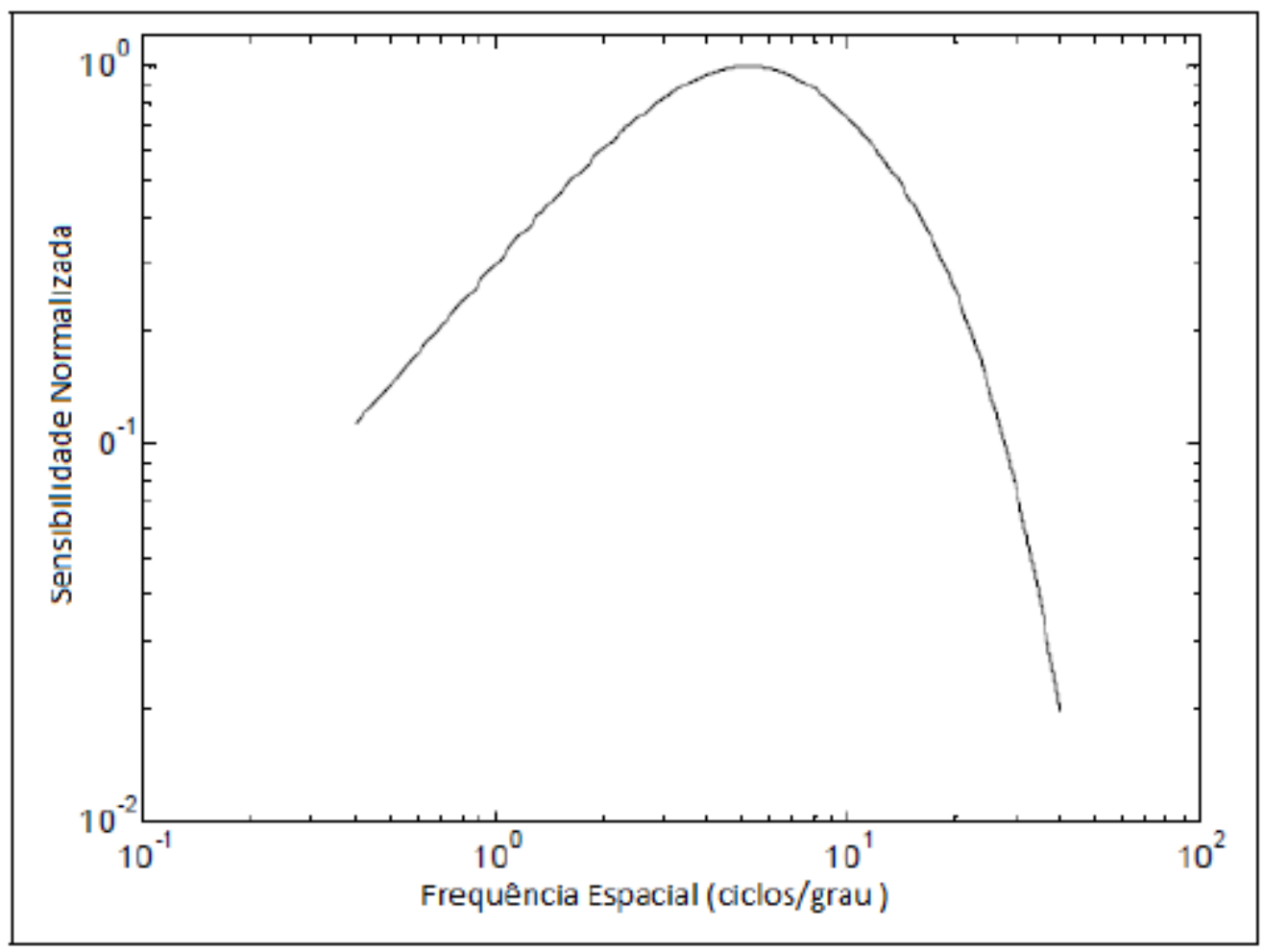

Figura A.2: Função de Sensibilidade ao Contraste (CSF) normalizada.

\section{- Percepção do Brilho}

A percepção humana ao brilho é referida como luminosidade, representada por $L^{*}$ é definida pela Comissão Internacional de Iluminação (CIE - Commission internationale de l'éclairage) conforme a equação:

$$
\begin{aligned}
& L^{*}=116 .\left(\frac{Y}{Y_{n}}\right)^{\frac{1}{3}}-16 \\
& \text { para } \frac{Y}{Y_{n}}>0.00856
\end{aligned}
$$


em que $Y$ é a raíz cúbica da luminância e $Y_{n}$ é uma referência. $L^{*}$ varia entre 0 e 100 e uma unidade sua representa o limiar da percepção humana para uma dada diferença de luminosidade [POYNTON, 1996]. Na Figura A.3 é mostrada a curva de transferência característica para $L^{*}$ em função de $Y$.

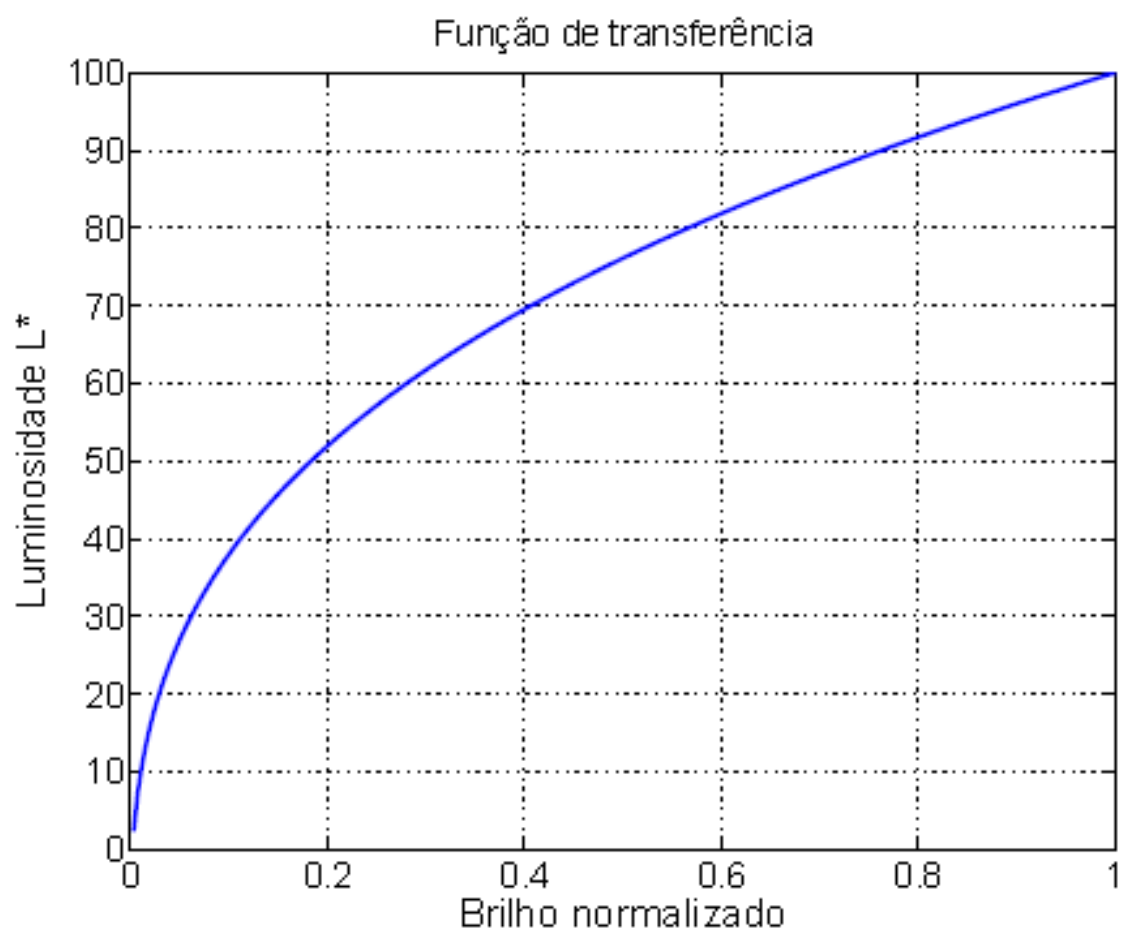

Figura A.3: Curva de transferência característica para $L^{*}$ em função de $Y$.

\section{- Percepção de Cores}

O olho humano possui três tipos diferentes de cones S (Short), M (Medium)e L (Long), sensíveis a diferentes comprimentos de onda: Curtos são sensíveis às luzes azuladas, médios são sensíveis às luzes esverdeadas e longos são sensíveis às luzes avermelhadas. A Figura A.4 representa a sensibilidade espectral dos três tipos de cone e do bastonete da retina humana. 


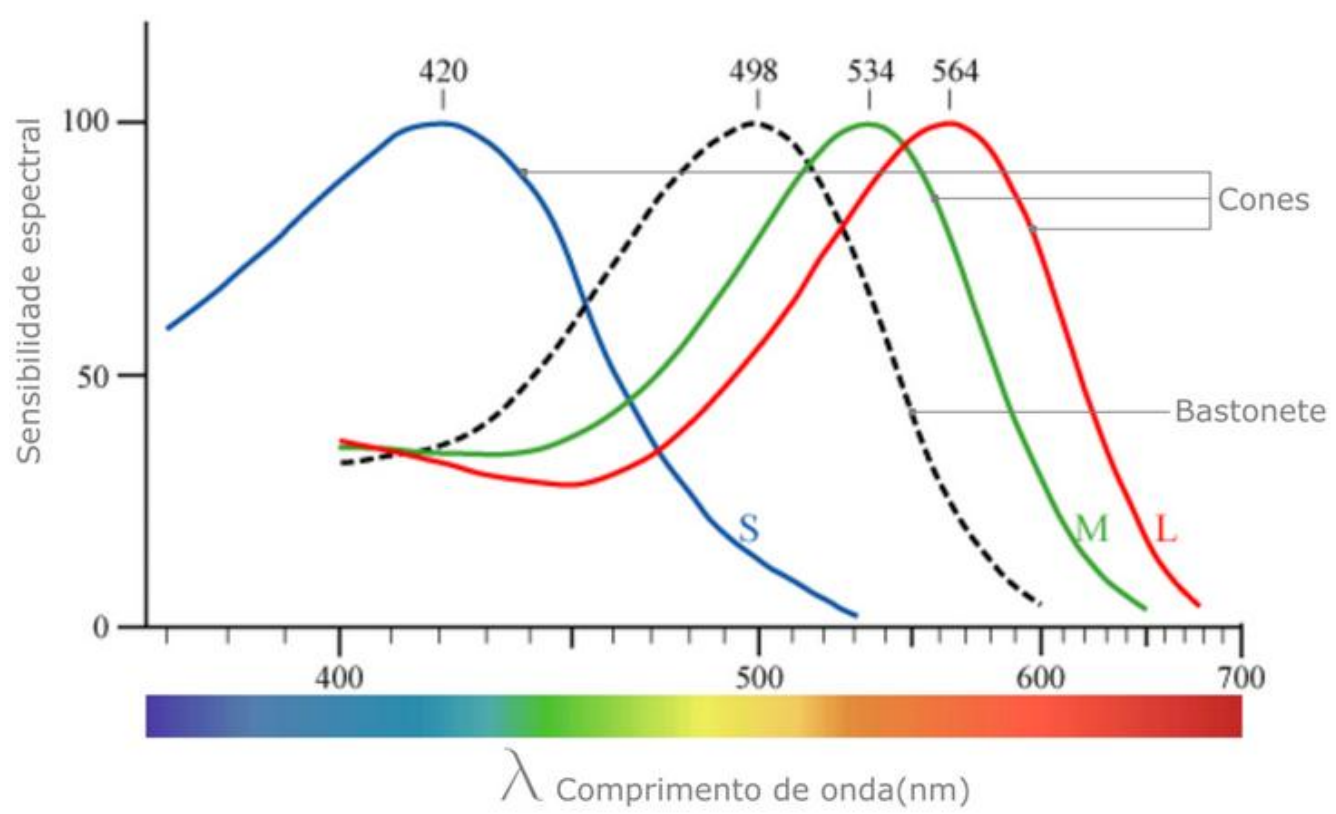

Figura A.4: Sensibilidade espectral dos cones (S,M,L) e do bastonete. 


\section{Referências Bibliográficas}

BASET, S.; H. G. SCHULZRINE. An analysis of the Skype peer-to-peer Internet telephony protocol. 25th IEEE International Conference on Computer Communications INFOCOM 2006. Barcelona: IEEE Conference Publications, 2006. p. 1-11.

BOVIK, A. C., Z. WANG, H. R. SHEIKH. Objective video quality assessment. In: Tha Handbook of Video Databases: Design and Applications. CRC Press, p. 1041-1078, Florida, 2003.

BRUNET, D., E. R. VRSCAY, e Z. WANG. On the Mathematical Properties of the Structural Similarity Index. IEEE Transactions on Image Processing, 2012. p 1488-1499.

BT.500-13, ITU-R Rec. Methodology for the subjective assessment of the quality of television pictures. January, 2012. Disponível em: <www.itu.int/rec/R-REC-BT.500/en〉.

CAIN, B., S. DEERING, B. FENNER, I. KOUVELAS, e A. THYAGARAJAN. Internet Group Management Protocol, Version 3. IETF. October, 2002. Disponível em $<$ http://tools.ietf.org/pdf/rfc3376.pdf>.

CAMPBELL, F. W., MAFFEI, L. Contrast and spatial frequency. Scientific American, v. 231, p. 106-114, 1974.

CASNER, S., e V. JACOBSON. Compressing IP/UDP/RTP Headers for Low-Speed Serial Links. IETF. February, 1999. Disponível em <https://tools.ietf.org/rfc/rfc2508.txt>.

CHEN, D., T. LIN, e S. HSIEH. A Transition-Aware DVS Method for Jitter-Controlled RealTime Scheduling. International Conference on Parallel and Distributed Computing, Applications and Technologies. Hiroshima: IEEE Conference Publications, 2009. p. 34-41.

COAQUIRA, D., ZEGARRA, D., e ARJONA, M. Avaliação de qualidade de vídeo sobre uma rede IP usando métricas objetivas. Revista Iberoamericana em Sistemas, Cibernética é Informática, 2011. p. 25-29. 
CORNSWEET, T. N. Visual Perception. Academic Press, Harcout, 1970. 475p.

DEERING, S., D. L. ESTRIN, D., JACOBSON, V. FARINACCI, C. G. LIU, e L. WEI. The PIM architecture for wide-area multicasting routing. IEEE ACM Transactions, 1996. p. 153162.

DWARAKA, V., e S. KILARI. Effect of Delay/Delay Variable on QoE in Video Streaming. Karlskrona: Blekinge Institute of Technology, 2010.

FONSECA, R., Algoritmos para Avaliação da Qualidade de Vídeo em Sistemas de Televisão Digital. São Paulo, 2008.

HARTE, L. The Introduction to MPEG: MPEG-1, MPEG-2 and MPEG-4. ALTHOS Publishing, North Carolina, 2006.

HOFFMAN, D., G. FERNANDO, V. GOYAL, e M. CIVANLAR. RTP Payload Format for MPEG1/MPEG2 Video. IETF. January, 1998. Disponível em: $<$ http://www.ietf.org/rfc/rfc2250.txt>.

ICKIN, S., K. DE VOGELEER, M. FIEDLER, e D. ERMAN. The Effects of Packet Delay Variation on the Perceptual Quality of Video. IEEE 35th Conference on Local Computer Networks (LCN). Denver: IEEE, 2010. p. 663-668.

J.144, ITU-T Rec. Objective perceptual video quality measurement techniques for digital cable television in the presence of a full reference. July, 2009. Disponível em: <http://www.itu.int/rec/T-REC-J.144-200403-I/>.

JAGADISH, S., e R. MANIVASAKAN. Analysis of jitter control algorithms in QoS networks. 2011 Second Asian Himalayas International Conference on Internet (AH-ICI). Kathmundu: IEEE Conference Publications, 2011. p. 1-5.

KIKUCHI, Y., T. NOMURA, S. FUKUNAGA, Y. MATSUI, e H. KIMATA. RTP Payload Format for MPEG-4 Audio/Visual Streams. IETF. November, 2000. Disponível em: $<$ http://tools.ietf.org/pdf/rfc3016.pdf>.

KLAUE, J., B. RATHKE, and A. WOLISZ. EvalVid - A Framework for Video Transmission and Quality Evaluation. Proceedings of 13th International Conference on Modelling, Techniques and Tools for Computer Performance Evaluation. Illinois: Springer, 2003. p. 255-272. 
KUROSE, F., e K. W. ROSS. Computer Networking: A Top-Down Approach. AddisonWesley, 2010.

MOY, J. Multicast Extension to OSPF. IETF. March, 1994. Disponível em: $<$ https://tools.ietf.org/rfc/rfc1584.txt>.

MSU Graphics \& Media Laboratory. MSU Video Quality Measurements tools. 2011. Disponível em: <http://compression.ru/video/quality_measure/index_en.html>.

NETEM. Network Emulation. 5 de October, 1991. Disponível em: <http://www.linuxfoundation.org/>.

NISHIDA, S. M. Sentido da Visão. Apostila do Curso de Fisiologia, 2007. Disponível em: $<$ http://www.ibb.unesp.br/>.

ODOM, W. CCENT/CCNA ICDN1, Official Exam Certification Guide. Cisco Systems, 2007. ODOM, W. CCENT/CCNA ICDN2, Official Exam Certification Guide. Cisco Systems, 2007.

ORTEGA, A., e K. RAMCHANDRAN. Rate-Distortion Methods for Image and Video Compression. IEEE Signal Processing Magazine, 2008. p. 23-50.

P.910, ITU-T Rec. Subjective video quality assessment methods for multimedia applications. April, 2008. Disponível em: 〈www.itu.int/rec/T-REC-P.910〉.

P.930, ITU-T Rec. Principles of a reference impairment system for video. August, 1996. Disponível em: <http://www.itu.int/rec/T-REC-P.930/>.

PARK, H., e D. HAR. Subjective Image Quality Assessment based on Objective Image Quality Measurement Factors. IEEE Transactions on Consumer Electronics, 2011. p. 11761184.

POYNTON, C. A. A Technical Introduction to Digital Video. New York: John Wiley \& Sons, 1996.

RICHARDSON, I. H.264 and MPEG-4 Video Compression. London: Wiley, 2003.

SANTOS, N. A., SIMAS, M. L. B. Função de sensibilidade ao contraste: Indicador da percepção visual da forma e da resolução espacial. Psicología: Reflexão e Crítica, v. 14, n. 3, 2011. p. 589-597. 
SCHULZRINNE, H., e S. CASNER. RTP Profile for Audio and Video Conferences with Minimal Control. IETF. July, 2003. Disponível em: 〈http://www.ietf.org/rfc/rfc3551.txt>.

SCHULZRINNE, H., S. CASNER, R. FREDERICK, e V. JACOBSON. RTP: A Transport Protocol for Real-Time Applications. IETF. July, 2003. Disponível em: $<$ http://tools.ietf.org/pdf/rfc3550.pdf >.

VAN DER MEER, J., D. MACKIE, V. SWAMINATHAN, D. SINGER, e P. GENTRIC. RTP Payload Format for Transport of MPEG-4 Elementary Streams. IETF. November, 2003. Disponível em: <http://tools.ietf.org/pdf/rfc3640.pdf>.

VLC, VideoLAN Project. Video Lan. 2001. Disponível em: <http://www.videolan.org/>.

VQEG, Video Quality Experts Group. Final Report from the Video Quality Experts Group on the Validation of Objective Models of Video Quality Assessment. March, 2000. Disponível em: <ftp://vqeg.its.bldrdoc.gov/>.

WAITZMAN, D., C. PARTRIDGE, e S. DEERING. Distance Vector Routing Multicast Protocol (DVRMP). IETF. November, 1988. Disponível em: <http://tools.ietf.org/pdf/rfc1075.pdf>.

WANG, Z., A. BOVIK, e H. SHEIKH. Image quality assessment: from error visibility to structural similarity. IEEE Transactions on Image Processing, 2004. p. 600-612.

WINKLER, S. Digital Video Quality: Vision Models and Metrics. Wiley, 2005.

WOLF, S., e M. H. PINSON. A New Standardized Method for Objectively Measuring Video Quality Abstract. IEEE Transactions on Broadcasting, 2004. p. 312-322.

XIAO, F. DCT-based Video Quality Evaluation. Stanford University, 2000. Disponível em: <http://www.compression.ru/video/quality_measure/vqm.pdf>.

XPIH. Xiph.org Video Test Media [derf's collection]. April, 2010. Disponível em: <http://media.xiph.org/video/derf/>.

Y.1540, ITU-T REC. Internet protocol data communication service - IP packet transfer and availability performance parameters. ITU. March, 2011. Disponível em: <http://www.itu.int/rec/T-REC-Y.1540/>. 
ZHANG, L., L. ZHENG, e K. NGEE. Effect of delay and delay jitter on voice/video over IP. Computer Communications, 2002. p. 863-873. 